\title{
Gravitational action with null boundaries
}

\author{
Luis Lehner, ${ }^{1}$ Robert C. Myers,${ }^{1}$ Eric Poisson ${ }^{2}$ and Rafael D. Sorkin ${ }^{1}$ \\ ${ }^{1}$ Perimeter Institute for Theoretical Physics, Waterloo, Ontario N2L 2Y5, Canada \\ ${ }^{2}$ Department of Physics, University of Guelph, Guelph, Ontario N1G 2W1, Canada
}

\begin{abstract}
We present a complete discussion of the boundary term in the action functional of general relativity when the boundary includes null segments in addition to the more usual timelike and spacelike segments. We confirm that ambiguities appear in the contribution from a null segment, because it depends on an arbitrary choice of parametrization for the generators. We also show that similar ambiguities appear in the contribution from a codimension-two surface at which a null segment is joined to another (spacelike, timelike, or null) segment. The parametrization ambiguity can be tamed by insisting that the null generators be affinely parametrized; this forces each null contribution to the boundary action to vanish, but leaves intact the fredom to rescale the affine parameter by a constant factor on each generator. Once a choice of parametrization is made, the ambiguity in the joint contributions can be eliminated by formulating well-motivated rules that ensure the additivity of the gravitational action. Enforcing these rules, we calculate the time rate of change of the action when it is evaluated for a so-called "Wheeler-deWitt patch" of a black hole in asymptotically-anti de Sitter space. We recover a number of results cited in the literature, obtained with a less complete analysis.
\end{abstract}

\section{CONTENTS}

I. Introduction and summary

II. Volume, surface, and joint contributions to the gravitational action 6

A. Background and previous results

\begin{tabular}{lr} 
B. Spacelike/timelike segment & 7 \\
\hline
\end{tabular}

1. Preliminaries $\quad 7$

2. Variation of geometric quantities

3. Boundary term

\begin{tabular}{lr} 
C. Null segment & 10 \\
\hline
\end{tabular}

1. Preliminaries

2. Variation of geometric quantities

3. Boundary term 12

4. Reparametrizations 14

5. Redefinition of $\Phi$

D. Closed hypersurface: Timelike and spacelike segments 14

E. Rules for timelike and spacelike joints

F. Closed hypersurface: Null and spacelike segments $\quad[19$

1. Past light cone truncated by spacelike segments $\quad 19$

2. Future light cone truncated by spacelike segments

3. Past and future light cone truncated by spacelike segments 21

4. Proof that $r_{a} \delta A^{a}= \pm \delta a$ and $\delta a=\delta \ln (-n \cdot k)$

G. Rules for null joints

H. Additivity rules

III. Rate of change of the gravitational action for AdS black holes

A. Schwarzschild-anti de Sitter spacetime 28

\begin{tabular}{ll} 
B. Wheeler-deWitt patch & 29 \\
\hline
\end{tabular}

C. Calculation of $\delta S \quad 30$

D. Comparison with Brown et al

E. Extension to charged black holes

\begin{tabular}{lr} 
IV. Discussion & 36 \\
\hline
\end{tabular}

Acknowledgments 38 
A. Ambiguities in the null limit from timelike surfaces 39

1. Static, spherically-symmetric spacetime 39

2. Kerr spacetime 41

3. Vaidya spacetime 41

B. Counterterm for the null boundary action 42

C. Action User's Manual 43

References $\quad 44$

\section{INTRODUCTION AND SUMMARY}

The action functional for the gravitational field in general relativity, the famous Hilbert-Einstein action, is given simply (in the absence of a cosmological constant) by the spacetime integral of the Ricci scalar. But it has long been recognized that a well-defined variational principle for a finite domain of spacetime must also involve a contribution from the domain's boundary [1, 2]. In the typical context in which the boundary consists of timelike and spacelike hypersurfaces, the boundary action is given by the surface integral of the trace of the extrinsic curvature. When the intersection between two segments of the boundary is not smooth, the extrinsic curvature is singular and the boundary action acquires additional contributions from the intersection [3, 4]. While all this is well-known, the case in which the boundary includes segments of null hypersurfaces has received very little attention in the literature. Indeed, to our knowledge the contribution of a null boundary to the gravitational action has only been examined recently in Refs. 5 . and [6]; the second reference, in particular, offers a detailed account of the variational principle of general relativity in the presence of null boundaries. But these works do not consider the contribution to the gravitational action coming from a nonsmooth intersection between a null segment of the boundary with another (spacelike, timelike, or null) segment. It appeared important to us to fill this gap, and to provide a complete account of the boundary term in the action functional of general relativity when the boundary includes null segments, in addition to the more usual timelike and spacelike segments.

The desire for completeness was not the sole motivation for undertaking this work. We were also motivated by a desire to better understand the calculations supporting the recent "complexity equals action" conjecture of Brown et al [7, 8, which was made in the context of the AdS/CFT correspondence [9]. This proposal emerged from previous studies attempting to understand the growth of the Einstein-Rosen bridge for AdS black holes in terms of circuit complexity in the dual boundary CFT [10 13. As we will describe, the calculations on the gravity side which support this conjecture rely in an essential way on evaluating the gravitational action for regions with null boundaries. On the CFT side, the conjecture considers the complexity $C$ of the quantum state $|\psi(t)\rangle$ on a particular time slice of the boundary conformal field theory. Loosely, we may think of $C$ as the minimum number of quantum gates required to produce $|\psi\rangle$ from a particular reference state - see [8] for further details. The conjecture then relates $C$ to the gravitational action $I$ evaluated for a corresponding region in the dual (asymptotically) anti-de Sitter spacetime, known as a "Wheeler-deWitt (WdW) patch." The WdW patch is the region enclosed by past and future light sheets sent into the bulk spacetime from the time slice on the boundary, where $C$ is to be evaluated. An example is illustrated in Fig. 1, and the conjecture states that $C=I /(\pi \hbar)$.

A particularly interesting case in which to examine this conjecture is that of an eternal black hole in anti de Sitter space [7, 8. In this case, the quantum state $\left|\psi\left(t_{\mathrm{L}}, t_{\mathrm{R}}\right)\right\rangle$ depends on two times $t_{\mathrm{L}}$ and $t_{\mathrm{R}}$, i.e., the time on each of the asymptotic boundaries on either side of the Einstein-Rosen bridge (the left and right boundaries in a conformal diagram) 17 The corresponding WdW patch is displayed in Fig. 1. In part, the "complexity equals action" conjecture was motivated by the expectation that the complexity in this situation should increase linearly in time (for a very long initial period), and the observation that this property is shared by the action of the Wheeler-deWitt patch. In particular, it was found that at late times [7, 8]

$$
\frac{d I}{d t}=2 M
$$

for a Schwarzschild-AdS black hole, where $M$ is the total mass-energy of the spacetime, and $t$ stands for one of the boundary times (i.e., $t_{\mathrm{L}}$ or $t_{\mathrm{R}}$ ), with the other time being held fixed. Similar results for other spacetimes, all indicating that $I$ increases linearly with $t$ at late times, were reported by Brown et al in support of the conjecture.

\footnotetext{
1 The two boundaries of an eternal AdS black hole correspond to the original CFT and its thermofield double, and the bulk geometry is then dual to a purification of a thermal density matrix involving these two CFTs [14].
} 


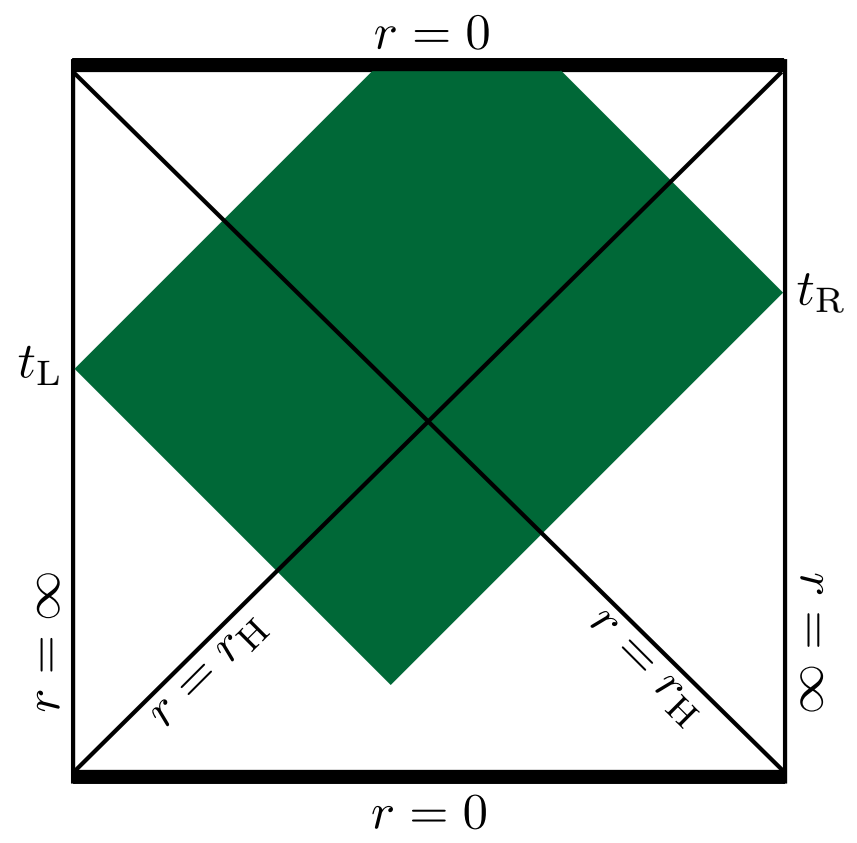

FIG. 1. Wheeler-deWitt patch of an eternal Schwarzschild-anti de Sitter black hole. The patch is defined by a future light cone originating inside the white-hole horizon and reaching the left boundary at time $t_{\mathrm{L}}$ and the right boundary at time $t_{\mathrm{R}}$. This light cone is joined to a past light cone converging to the future singularity.

One of our main purposes in this paper is to critically examine how Eq. 1.1 was obtained: We question the methods by which $d I / d t$ was calculated in [7, 8, we identify what we take to be a more rigorous approach, and we recalculate $d I / d t$ according to these methods. We perform the calculations for both a Schwarzschild and ReissnerNorström black hole in anti-de Sitter space, and we find that our results for $d I / d t$ precisely agree with those reported by Brown et al [7, 8. This agreement, in spite of the very different methods used in the calculation, may seem at first sight a surprising outcome. The mechanism behind the agreement will be discussed in detail in Sec. IIID.

There are two main reasons to suspect the methods adopted by Brown et al, and hence to be skeptical of their results for $d I / d t$. First, the Wheeler-deWitt patch has a boundary that includes segments of null hypersurfaces, and the familiar boundary term in the gravitational action (the Gibbons-Hawking-York $K$ term [1, 2]) is ill-defined for such segments; it applies only to spacelike or timelike segments of the boundary. One might attempt to evade this problem by evaluating the boundary contribution for a null segment by approaching the hypersurface through a sequence of timelike or spacelike surfaces. However, as we will show in Appendix A in general this limiting procedure is ambiguous and does not yield a unique answer. The second key problem is that the boundary of the Wheeler-deWitt patch also includes codimension-two surfaces — joints - at which different boundary surfaces intersect; for example, in Fig. 1. the spacelike portion of the boundary at (or rather near) $r=0$ is joined to null segments extending towards the two asymptotic AdS regions. Because the boundary is not smooth at such joints, we should expect them to make separate contributions to the gravitational action, in spite of the higher codimensionality of these surfaces. Indeed, that nonsmooth portions of the boundary contribute to the action was demonstrated by Hayward 4] in the case of joints between timelike and spacelike surfaces ${ }^{2}$ One might attempt to define the joint contributions for an intersection involving a null segment by applying a limiting procedure to the Hayward terms, but as we show in Appendix A this yields a divergent result.

The first issue, of correctly assigning a boundary contribution to the gravitational action when the boundary includes a null segment, was recently examined by Neiman 5 and given a much more thorough analysis by Parattu et al 6 . The correct boundary term is identified by a careful consideration of the variational principle for general relativity, which keeps track of all terms that are pushed to the boundary when an integration by parts is carried out. In the case of a timelike or spacelike segment, this exercise reveals the Gibbons-Hawking-York $K$ term, i.e., the trace of the extrinsic curvature integrated over the boundary segment [1, 2]. In the case of a null segment, Parattu et al show

\footnotetext{
${ }^{2}$ Similar joint contributions were found for the Regge calculus action with Euclidean signature in 3 .
} 
that the boundary term is given by an integral of the form ${ }^{3} \int \kappa d S d \lambda$, in which $\lambda$ is the parameter running on the null generators of the hypersurface, $d S$ is an area element on the cross-sections $\lambda=$ constant, and $\kappa(\lambda)$ measures the failure of $\lambda$ to be an affine parameter ${ }^{4}$ if the vector field $k^{\alpha}$ is tangent to the null generators, then $k^{\beta} \nabla_{\beta} k^{\alpha}=\kappa k^{\alpha}$. This expression for the boundary term reveals a striking fact: its value depends on the parametrization of the null generators, and it can be altered at will by a change of parametrization. For example, the boundary term vanishes when $\lambda$ is chosen to be an affine parameter. This observation implies that in general, the gravitational action is ambiguous when it is evaluated for a region of spacetime that is bounded in part by a segment of null hypersurface.

The second issue, the proper accounting of contributions from joints, was examined by Hayward 4 in the context of timelike and spacelike surfaces, but his treatment does not apply to null surfaces. We consider such situations in this paper, and evaluate the contribution of null joints to the gravitational action. We recall that Hayward's conclusion was that when (say) two spacelike segments are joined together, the boundary term in the action acquires a contribution of the form $\int \eta d S$, where $\eta$ is the rapidity parameter relating the two unit normals by a Lorentz transformation, and $d S$ is a surface element on the joint. On the other hand, when a spacelike, timelike, or null segment is joined to a null segment of the boundary, we find below that the contribution to the boundary action is of the similar form $\int a d S$, where $a$ is a quantity tied to the description of the null hypersurface ${ }^{5}$ More precisely, if the null segment is described by the equation $\Phi=0$, with $\Phi$ a scalar function in the spacetime, and if its null normal is given by $k_{\alpha}=-\mu \partial_{\alpha} \Phi$ in the adopted parametrization, with $\mu$ another scalar, then $a=\ln \mu$. This contribution to the action is also ambiguous, because $a$ can be changed at will by a redefinition of the function $\Phi$.

These observations imply that the computation of the gravitational action for a Wheeler-deWitt patch is plagued with ambiguities: The contribution to the action from each null segment of the boundary depends arbitrarily on the choice of parametrization for the generators, and the contribution from each joint between a null segment and another (spacelike, timelike, or null) segment is also arbitrary. These ambiguities may seem to be problematic for the "complexity equals action" conjecture, but we note that the complexity is also expected to be ambiguous - we return to this point in Sec. IV. In any event, at a pragmatic level, the ambiguities must be tamed before $I$ can be computed and featured in a critical examination of the conjecture. Let us add that the ambiguities apply only to the gravitational action evaluated for a given region of a given spacetime - the on-shell action; they are evaded when the action is varied in an implementation of the variational principle for general relativity.

To see in more concrete terms what the gravitational action looks like when it is evaluated for a region $\mathscr{V}$ of spacetime whose boundary $\partial \mathscr{V}$ is broken up into a number of segments, we consider (in some fixed spacetime) the region illustrated in Fig. 2. The boundary includes four spacelike segments, four null segments, and the joints between them. For this region, the gravitational action takes the form of

$$
S:=16 \pi G_{\mathrm{N}} I=\int_{\mathscr{V}}(R-2 \Lambda) d V+S_{\partial \mathscr{V}},
$$

where $G_{\mathrm{N}}$ is Newton's gravitational constant, $R$ the Ricci scalar, $\Lambda$ the cosmological constant, $d V$ an invariant volume element in $\mathscr{V}$, and where the boundary term is given explicitly given by

$$
\begin{aligned}
S_{\partial \mathscr{V}}= & 2 \int_{\mathcal{S}_{1}} K d \Sigma+2 \int_{\mathcal{S}_{2}} K d \Sigma-2 \int_{\mathcal{S}_{3}} K d \Sigma-2 \int_{\mathcal{S}_{4}} K d \Sigma \\
& +2 \int_{\mathcal{N}_{1}} \kappa d S d \lambda+2 \int_{\overline{\mathcal{N}}_{2}} \kappa d S d \lambda-2 \int_{\overline{\mathcal{N}}_{3}} \kappa d S d \lambda-2 \int_{\mathcal{N}_{4}} \kappa d S d \lambda \\
& +2 \oint_{\mathcal{B}_{11}} a d S-2 \oint_{\mathcal{B}_{12}} \eta d S+2 \oint_{\mathcal{B}_{22}} a d S-2 \oint_{\mathcal{B}_{24}} a d S \\
& +2 \oint_{\mathcal{B}_{44}} a d S+2 \oint_{\mathcal{B}_{34}} \eta d S+2 \oint_{\mathcal{B}_{33}} a d S-2 \oint_{\mathcal{B}_{13}} a d S,
\end{aligned}
$$

in terms of quantities introduced previously. The sign in front of each integral will be explained in the technical sections of the paper. We recall that the contribution from each null segment is ill-defined because it depends on the choice of $\lambda$ (which implies a choice of $\kappa$ ), and that except for $\mathcal{B}_{12}$ and $\mathcal{B}_{34}$, the contribution from each joint is also ill-defined because of the freedom to redefine $a$.

The gravitational action only becomes well-defined when rules are introduced to specify $\kappa(\lambda)$ on each null segment, and $a$ on each null joint. An attractive choice of parametrization suggests itself: By ensuring that the generators of

\footnotetext{
3 These authors also include a term involving the expansion $\Theta$ of the null generators. As we discuss below, this term is not required because it depends only on the surface's intrinsic geometry.

4 This can also be interpreted as (a component of) the extrinsic curvature of the null segment [15] 16].

5 As this paper was nearing completion, we learned that similar junction terms were proposed in [17].
} 


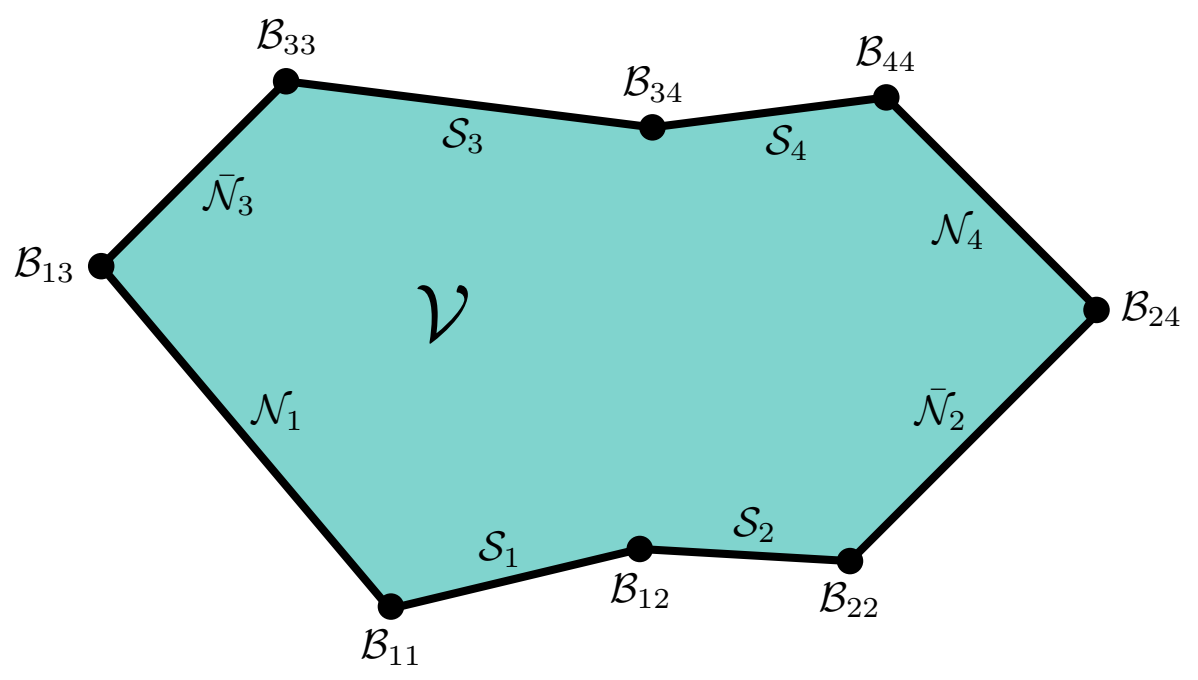

FIG. 2. A region $\mathscr{V}$ of spacetime with its broken boundary $\partial \mathscr{V}$. The boundary consists of four spacelike segments $\mathcal{S}_{1}, \mathcal{S}_{2}, \mathcal{S}_{3}$, $\mathcal{S}_{4}$, and four null segments $\mathcal{N}_{1}, \overline{\mathcal{N}}_{2}, \overline{\mathcal{N}}_{3}, \mathcal{N}_{4}$. These are joined at codimension-two surfaces denoted $\mathcal{B}_{j k}$.

each null segment are affinely parametrized, we can set $\kappa=0$ and eliminate all such contributions to $S_{\partial \mathscr{V}}$. A wellmotivated rule to assign $a$ on each null joint emerges when the influence of these joints on the variational principle is duly considered, and when the on-shell gravitational action is required to be properly additive, in the sense that $S(\mathscr{V})=S\left(\mathscr{V}_{1}\right)+S(\mathscr{V} / 2)$ when a spacetime region $\mathscr{V}$ is subdivided into two subregions, $\mathscr{V}_{1}$ and $\mathscr{V} 2.6$ Our rule goes as follows: When a null segment is joined to a spacelike or timelike surface, we set $a=\ln |n \cdot k|$, where $n^{\alpha}$ is the unit normal to the spacelike or timelike surface, $k^{\alpha}$ is the normal to the null hypersurface, and $n \cdot k:=g_{\alpha \beta} n^{\alpha} k^{\beta}$ is their inner product; when a null segment is joined to another null segment, we set instead $a=\ln \left|\frac{1}{2} k \cdot \bar{k}\right|$, where $k^{\alpha}$ and $\bar{k}^{\alpha}$ are the null normals. Along with an appropriate specification of signs, this rule ensures that the on-shell gravitational action is properly additive 7

The assignment $\kappa=0$ and the joint rules for $a$ eliminate the ambiguities from the gravitational action, except for a remaining freedom to rescale $\lambda$ by a constant factor. We can remove this final ambiguity for the WdW patch by imposing a normalization condition on the null normals at the asymptotic AdS boundary. With these rules in place, we can finally turn to the task of evaluating $S$ for a Wheeler-deWitt patch of a Schwarzschild-AdS black hole, and calculate its rate of change $d S / d t$. As stated previously and described in detail below, we arrive at precisely the same result first obtained by Brown et al $\left[\underline{8}: d S / d t=32 \pi G_{\mathrm{N}} M\right.$, or $d I / d t=2 M$.

In the remainder of the paper we offer a detailed account of the results summarized above. We begin in Sec. II with a thorough description of the variational principle in general relativity, when $\partial \mathscr{V}$ consists of spacelike, timelike, and null hypersurface segment: 8 , After some preliminary remarks in Sec. II A we review the well-understood case of timelike and spacelike segments in Sec. IIB, before moving on to the null case in Sec. IIC. In Sec. IID we form a closed boundary by joining spacelike and timelike segments, and obtain Hayward's expression for the $\eta$-terms in the gravitational action; his rules are summarized in Sec. IIE. In Sec. IIF we form a closed boundary by joining spacelike and null segments, and derive the appropriate joint contributions to the action, our own $a$-terms; the rules for null joints are summarized in Sec. IIG. The additivity rules for $a$ are formulated in Sec. IIH] which concludes the section. While much of the material contained in Sec. III is known from the literature, we consider that a self-contained and complete account enhances the clarity of the presentation. We also take the opportunity to fill in some of the technical details left implicit in Hayward's work [4, and to provide minor improvements on the developments of Parattu et al 6].

In Sec. III we revisit the calculation of $d S / d t$ for a Wheeler-deWitt patch of a spacetime describing an eternal black hole in asymptotically anti-de Sitter space. We begin with an uncharged black hole in Secs. IIIA, IIIB, and IIIC compare our calculations to those of Brown et al [8] in Sec. IIID, and turn to the charged case in Sec. IIIE. In

${ }^{6}$ Additivity beyond the on-shell action, for example in the context of a path integral for quantum gravity, cannot be guaranteed. This is because the metric may not be sufficiently smooth across a null boundary to ensure that $\lambda$ is an affine parameter on both sides of the boundary. A further obstruction to addivity would arise from an extra imaginary contribution to the action, which has been argued to exist in [18 20 - see also [5 21, 24]. However, this contribution is typically neglected and we do so here as well.

7 It should be noted that there are exceptions to this statement: As Brill and Hayward have demonstrated 25, the action may not be additive when a joint $\mathcal{B}$ possesses a timelike direction; we exclude such cases from our considerations.

8 A treatment by complementary methods will appear in 26$]$. 
Appendix A we provide an analysis of the ambiguous nature of the gravitational action when the boundary includes a null segment. We show that in general, evaluating the boundary action for a null segment through the limit of a sequence of (say) timelike surfaces produces an ill-defined result. A notable exception to this statement arises when the limit is a stationary null surface, e.g., a Killing horizon; in this case the limit is unique. The theme is pursued further in Appendix B, in which we show that the parametrization ambiguity of the gravitational action can be eliminated by adding a suitable counterterm; the consequences of this observation will be explored in a forthcoming publication [27. Finally, we conclude in Appendix C with an Action User's Manual that provides a concise guide on how each relevant contribution to the action, and in particular, the sign of each contribution, are evaluated.

\section{VOLUME, SURFACE, AND JOINT CONTRIBUTIONS TO THE GRAVITATIONAL ACTION}

For the developments of this section, we focus our attention on a spacetime of $d=4$ dimensions; the generalization to higher-dimensional spacetimes is immediate. To keep track of the sign of different contributions to the action, we adopt the convention according to which all timelike and null normal vectors are future-directed, and all spacelike normals are outward-directed with respect to the region of interest. Further, we recall that the quantity $S$ considered below is related to the usual gravitational action $I$ by $S=16 \pi G_{\mathrm{N}} I$, as defined in Eq. 1.2.

\section{A. Background and previous results}

It is well-known that the Hilbert-Einstein action defined on a four-dimensional domain $\mathscr{V}$,

$$
S_{\mathscr{V}}:=\int_{\mathscr{V}}(R-2 \Lambda) \sqrt{-g} d^{4} x,
$$

must be supplemented by a boundary term $S_{\partial \mathscr{V}}$ in order to give rise to a variational principle in which only the metric variation $\delta g^{\mu \nu}$ (and not its derivatives) is required to vanish on the boundary $\partial \mathscr{V}$. The reason is that variation of $S_{\mathscr{V}}$ produces the expression

$$
\delta S_{\mathscr{V}}=\int_{\mathscr{V}}\left(G_{\mu \nu}+\Lambda g_{\mu \nu}\right) \delta g^{\mu \nu} \sqrt{-g} d^{4} x+\oint_{\partial \mathscr{V}} \delta v^{\mu} d \Sigma_{\mu},
$$

with an additional boundary term that must be properly disposed of. We have introduced

$$
\delta v^{\mu}:=g^{\alpha \beta} \delta \Gamma_{\alpha \beta}^{\mu}-g^{\alpha \mu} \delta \Gamma_{\alpha \beta}^{\beta},
$$

and $d \Sigma_{\mu}$ is an outward-directed surface element on $\partial \mathscr{V}$. The slash in the middle of the $\delta$ symbol in $\delta v^{\mu}$ reminds us that this infinitesimal quantity is not the variation of another quantity $v^{\mu}$.

The manipulations carried out in the following subsections reveal that in all the cases considered,

$$
\oint_{\partial \mathscr{V}} \delta v^{\mu} d \Sigma_{\mu}=-\delta S_{\partial \mathscr{V}}
$$

where $S_{\partial \mathscr{V}}$ is a suitable boundary term, whose variation reproduces the expression of the left-hand side when the induced metric on $\partial \mathscr{V}$ is held fixed. With this result established, the gravitational action is properly identified with $S_{\mathscr{V}}+S_{\partial \mathscr{V}}$, and its variation yields

$$
\delta\left(S_{\mathscr{V}}+S_{\partial \mathscr{V}}\right)=\int_{\mathscr{V}}\left(G_{\mu \nu}+\Lambda g_{\mu \nu}\right) \delta g^{\mu \nu} \sqrt{-g} d^{4} x .
$$

The boundary $\partial \mathscr{V}$ is usually constructed from spacelike and timelike hypersurface segments, and in this case the manipulations that lead to the identification of $S_{\partial \mathscr{V}}$ are well-known. The most complete version of this computation was presented by Hayward [4, who paid careful attention to situations in which $\partial \mathscr{V}$ is not a smooth hypersurface. Specifically, Hayward examined cases in which a (timelike or spacelike) segment of $\partial \mathscr{V}$ is joined to another (timelike or spacelike) segment at a two-dimensional surface, in such a way that the normal vector field is discontinuous at the joint. He showed that in general, such joints contribute to the boundary action. We reproduce Hayward's computations below, and provide details that were left out of his paper.

The boundary $\partial \mathscr{V}$ can also include segments of null hypersurfaces. This case was not given much attention in the literature, with the notable recent exceptions of Neiman [5] and Parattu et al. [6]. We revisit these constructions here, 
providing a more complete treatment. Unlike Neiman, who took the null generators of the hypersurface segments to be affinely parametrized, we allow the generators to be arbitrarily parametrized. This generalization reveals the important fact that the boundary action evaluated on a null segment depends on the choice of parameter and is therefore ill-defined in general. And unlike Parattu et al, who did allow for an arbitrary parametrization but did not consider the joints with other surfaces, we pay close attention to the joint that arises when a null segment is joined to a spacelike, timelike, or null segment. Our manipulations pertaining to a given null segment also offer a minor improvement on the treatment provided by Parattu et al: while their derivation requires the normal vector to $\partial \mathscr{V}$ to be given an extension off the hypersurface so as to define its derivatives in all directions, our derivation involves only tangential derivatives and therefore does not require such an extension.

\section{B. Spacelike/timelike segment}

We begin with the well-studied task of evaluating $\int \delta v^{\mu} d \Sigma_{\mu}$ on a spacelike or timelike segment of $\partial \mathscr{V}$. We denote this segment by $\Sigma$, and imagine that it is bounded by the two-surfaces $\mathcal{B}_{1}$ and $\mathcal{B}_{2}$. When $\Sigma$ is spacelike, $\mathcal{B}_{1}$ represents an inner boundary, and $\mathcal{B}_{2}$ an outer boundary. When $\Sigma$ is timelike, $\mathcal{B}_{1}$ represents a past boundary, and $\mathcal{B}_{2}$ a future boundary.

\section{Preliminaries}

We first import some helpful results from Secs. 3.1, 3.2, and 3.4 of Ref. 28. (We will make frequent use of results obtained in this book, and we shall refer to it as the Toolkit.) The hypersurface $\Sigma$ is described by the relation $\Phi\left(x^{\alpha}\right)=0$ for some scalar field $\Phi$. When $\Sigma$ is spacelike, $\Phi$ is taken to increase toward the future across the hypersurface; when it is timelike, $\Phi$ increases outward. The hypersurface can also be described by parametric equations $x^{\alpha}=x^{\alpha}\left(y^{a}\right)$, in which $y^{a}$ are intrinsic coordinates on the hypersurface. The unit normal is

$$
n_{\alpha}=\epsilon \mu \partial_{\alpha} \Phi
$$

where $\epsilon:=n_{\alpha} n^{\alpha}= \pm 1$ and $\mu:=\left|g^{\alpha \beta} \partial_{\alpha} \Phi \partial_{\beta} \Phi\right|^{-1 / 2}$. The equation implies that when $\Sigma$ is spacelike, $n^{\alpha}$ is a futuredirected vector; when $\Sigma$ is timelike, $n^{\alpha}$ points to the outside. The vectors

$$
e_{a}^{\alpha}:=\frac{\partial x^{\alpha}}{\partial y^{a}}
$$

are tangent to $\Sigma$ and orthogonal to $n_{\alpha}$. We define $e_{\alpha}^{a}:=h^{a b} g_{\alpha \beta} e_{b}^{\beta}$. The induced metric on the hypersurface is

$$
h_{a b}:=g_{\alpha \beta} e_{a}^{\alpha} e_{b}^{\beta},
$$

and we denote its determinant by $h$ and its inverse by $h^{a b}$. The completeness relation for the inverse metric is given by

$$
g^{\alpha \beta}=\epsilon n^{\alpha} n^{\beta}+h^{\alpha \beta}, \quad h^{\alpha \beta}:=h^{a b} e_{a}^{\alpha} e_{b}^{\beta} .
$$

The directed surface element on $\Sigma$ is

$$
d \Sigma_{\alpha}=\epsilon n_{\alpha} d \Sigma, \quad d \Sigma:=|h|^{1 / 2} d^{3} y,
$$

with the convention that $d \Sigma_{\alpha} \propto \partial_{\alpha} \Phi$, with a positive factor of proportionality. The extrinsic curvature of the hypersurface is defined by

$$
K_{a b}:=e_{a}^{\alpha} e_{b}^{\beta} \nabla_{\alpha} n_{\beta},
$$

and we recall the identity

$$
e_{a}^{\alpha} \nabla_{\alpha} e_{b}^{\beta}=\Gamma_{a b}^{c} e_{c}^{\beta}-\epsilon K_{a b} n^{\beta}
$$

for the derivatives of the tangent vectors; the Christoffel symbols $\Gamma_{a b}^{c}$ are those constructed from $h_{a b}$. 


\section{Variation of geometric quantities}

We next perform a variation $\delta g^{\alpha \beta}$ of the metric, and see how various geometric quantities defined on $\Sigma$ respond to the variation. In this exercise it is understood that the description of the hypersurface is unchanged during the variation, so that the equations $\Phi=0$ and $x^{\alpha}=x^{\alpha}\left(y^{a}\right)$ keep their original form. This implies that the tangent vectors $e_{a}^{\alpha}$ are unaffected by the variation. A variation of the metric, however, induces a variation of $n_{\alpha}$, which is given by

$$
\delta n_{\alpha}=\frac{\delta \mu}{\mu} n_{\alpha}, \quad \frac{\delta \mu}{\mu}=-\frac{1}{2} \epsilon n_{\alpha} n_{\beta} \delta g^{\alpha \beta} .
$$

There is also a change in $e_{\alpha}^{a}$ : the relation $\delta_{b}^{a}=e_{\alpha}^{a} e_{b}^{\alpha}$ implies that $0=e_{b}^{\alpha} \delta e_{\alpha}^{a}$, so that

$$
\delta e_{\alpha}^{a}=\delta A^{a} n_{\alpha}
$$

for some infinitesimal quantity $\delta A^{a}$. The relation $0=e_{\alpha}^{a} n^{\alpha}$ implies that $0=e_{\alpha}^{a} \delta n^{\alpha}+\epsilon \delta A^{a}$, and $\epsilon=n_{\alpha} n^{\alpha}$ implies that $0=n_{\alpha} \delta n^{\alpha}+\epsilon \delta \mu / \mu$. We have obtained

$$
\delta n^{\alpha}=-\frac{\delta \mu}{\mu} n^{\alpha}-\epsilon \delta A^{a} e_{a}^{\alpha}
$$

for the variation of $n^{\alpha}$.

The quantity $\delta A^{a}$ can be expressed in a number of ways. We have

$$
\delta A^{a}=\epsilon n^{\alpha} \delta e_{\alpha}^{a}=-\epsilon e_{\alpha}^{a} \delta n^{\alpha},
$$

and combining the second form with the identity $\delta n^{\alpha}=n_{\beta} \delta g^{\alpha \beta}+g^{\alpha \beta} \delta n_{\beta}$ gives

$$
\delta A^{a}=-\epsilon e_{\alpha}^{a} n_{\beta} \delta g^{\alpha \beta}
$$

This shows that $\delta A^{a}$ is associated with the variation of the normal-tangent components of the inverse metric. Further, these results reveal that $\delta A^{a}$ is not the variation of a quantity $A^{a}$.

The completeness relation for the inverse metric implies that

$$
\delta g^{\alpha \beta}=-2 \epsilon \frac{\delta \mu}{\mu} n^{\alpha} n^{\beta}-\delta A^{a}\left(e_{a}^{\alpha} n^{\beta}+n^{\alpha} e_{a}^{\beta}\right)+\delta h^{a b} e_{a}^{\alpha} e_{b}^{\beta} .
$$

This expression confirms that $\delta \ln \mu$ represents the variation of the normal-normal component of the inverse metric, $\delta A^{a}$ the variation of the normal-tangent components, and shows that the variation of the purely tangential components is captured by $\delta h^{a b}$.

We next work out two expressions for $\delta K$, the variation of the trace of the extrinsic curvature. For the first, we begin with $K=h^{a b} K_{a b}$ and write $\delta K=K_{a b} \delta h^{a b}+h^{a b} \delta K_{a b}$. Recalling the definition of the extrinsic curvature, we have that

$$
\begin{aligned}
\delta K_{a b} & =e_{a}^{\alpha} e_{b}^{\beta}\left(\nabla_{\alpha} \delta n_{\beta}-n_{\mu} \delta \Gamma_{\alpha \beta}^{\mu}\right) \\
& =e_{a}^{\alpha} e_{b}^{\beta}\left[\nabla_{\alpha}(\delta \ln \mu) n_{\beta}+(\delta \ln \mu) \nabla_{\alpha} n_{\beta}-n_{\mu} \delta \Gamma_{\alpha \beta}^{\mu}\right] \\
& =\frac{\delta \mu}{\mu} K_{a b}-e_{a}^{\alpha} e_{b}^{\beta} n_{\mu} \delta \Gamma_{\alpha \beta}^{\mu},
\end{aligned}
$$

so that

$$
\delta K=K_{a b} \delta h^{a b}+\frac{\delta \mu}{\mu} K-h^{\alpha \beta} n_{\mu} \delta \Gamma_{\alpha \beta}^{\mu}
$$

For the second expression for $\delta K$, we begin with $\delta K=h_{\beta}^{\alpha} \nabla_{\alpha} n^{\beta}$ which implies

$$
\delta K=\left(\delta h_{\beta}^{\alpha}\right) \nabla_{\alpha} n^{\beta}+h_{\beta}^{\alpha} \nabla_{\alpha} \delta n^{\beta}+n^{\alpha} h_{\mu}^{\beta} \delta \Gamma_{\alpha \beta}^{\mu} .
$$

To evaluate the first term, we write $h_{\beta}^{\alpha}=e_{a}^{\alpha} e_{\beta}^{a}$, take the variation to get $\delta h^{\alpha}{ }_{\beta}=e_{a}^{\alpha} n_{\beta} \delta A^{a}$, and combine this with $\nabla_{\alpha} n^{\beta}$ to get zero, because $e_{a}^{\alpha} n_{\beta} \nabla_{\alpha} n^{\beta}=\frac{1}{2} e_{a}^{\alpha} \nabla_{\alpha}\left(n_{\beta} n^{\beta}\right)=0$. The second term requires more work. We have

$$
h_{\beta}^{\alpha} \nabla_{\alpha} \delta n^{\beta}=e_{a}^{\alpha} e_{\beta}^{a} \nabla_{\alpha} \delta n^{\beta}
$$




$$
\begin{aligned}
& =e_{a}^{\alpha} \nabla_{\alpha}\left(e_{\beta}^{a} \delta n^{\beta}\right)-e_{a}^{\alpha}\left(\nabla_{\alpha} e_{\beta}^{a}\right) \delta n^{\beta} \\
& =-\epsilon \partial_{a} \delta A^{a}+\epsilon \delta A^{b} e_{a}^{\alpha} e_{b}^{\beta} \nabla_{\alpha} e_{\beta}^{a}+\frac{\delta \mu}{\mu} e_{a}^{\alpha} n^{\beta} \nabla_{\alpha} e_{\beta}^{a} .
\end{aligned}
$$

The second term involves

$$
e_{a}^{\alpha} e_{b}^{\beta} \nabla_{\alpha} e_{\beta}^{a}=e_{a}^{\alpha} \nabla_{\alpha}\left(e_{b}^{\beta} e_{\beta}^{a}\right)-e_{a}^{\alpha} e_{\beta}^{a} \nabla_{\alpha} e_{b}^{\beta}=e_{a}^{\alpha} \nabla_{\alpha}\left(\delta_{b}^{a}\right)-e_{\beta}^{a} \Gamma_{a b}^{c} e_{c}^{\beta}=-\Gamma_{c b}^{c},
$$

and the third term involves

$$
e_{a}^{\alpha} n^{\beta} \nabla_{\alpha} e_{\beta}^{a}=e_{a}^{\alpha} \nabla_{\alpha}\left(n^{\beta} e_{\alpha}^{a}\right)-e_{a}^{\alpha} e_{\beta}^{a} \nabla_{\alpha} n^{\beta}=-K .
$$

Collecting results, we have obtained

$$
h_{\beta}^{\alpha} \nabla_{\alpha} \delta n^{\beta}=-\epsilon D_{a} \delta A^{a}-\frac{\delta \mu}{\mu} K,
$$

where $D_{a}$ is the covariant-derivative operator compatible with the induced metric $h_{a b}$. Our second expression for $\delta K$ is therefore

$$
\delta K=-\epsilon D_{a} \delta A^{a}-\frac{\delta \mu}{\mu} K+n^{\alpha} h_{\mu}^{\beta} \delta \Gamma_{\alpha \beta}^{\mu} .
$$

\section{Boundary term}

We may now evaluate

$$
\int_{\Sigma} \delta v^{\mu} d \Sigma_{\mu}=\int_{\Sigma} \epsilon \delta v^{\mu} n_{\mu} d \Sigma
$$

when $\Sigma$ is a spacelike or timelike hypersurface. We have that

$$
\delta v^{\mu} n_{\mu}=\left(g^{\alpha \beta} n_{\mu}-n^{\alpha} \delta^{\beta}{ }_{\mu}\right) \delta \Gamma_{\alpha \beta}^{\mu}=\left(h^{\alpha \beta} n_{\mu}-n^{\alpha} h^{\beta}{ }_{\mu}\right) \delta \Gamma_{\alpha \beta}^{\mu},
$$

where the completeness relation was used to go from the first expression to the second. Invoking next Eqs. (2.20) and (2.26), we obtain

$$
\delta v^{\mu} n_{\mu}=-2 \delta K-\epsilon D_{a} \delta A^{a}+K_{a b} \delta h^{a b},
$$

so that

$$
\int_{\Sigma} \delta v^{\mu} d \Sigma_{\mu}=\int_{\Sigma} \epsilon\left(-2 \delta K+K_{a b} \delta h^{a b}\right) d \Sigma-\oint_{\mathcal{B}_{2}} \delta A^{a} d S_{a}+\oint_{\mathcal{B}_{1}} \delta A^{a} d S_{a},
$$

where $d S_{a}$ is a surface element on $\mathcal{B}_{1}$ and $\mathcal{B}_{2}$, the boundaries of $\Sigma$.

We now require the variation $\delta h^{a b}$ to vanish on $\Sigma 9$ and see that the former expression becomes

$$
\begin{aligned}
\int_{\Sigma} \delta v^{\mu} d \Sigma_{\mu} & =-2 \epsilon \int_{\Sigma} \delta K d \Sigma-\oint_{\mathcal{B}_{2}} \delta A^{a} d S_{a}+\oint_{\mathcal{B}_{1}} \delta A^{a} d S_{a} \\
& =\delta\left(-2 \epsilon \int_{\Sigma} K d \Sigma\right)-\oint_{\mathcal{B}_{2}} \delta A^{a} d S_{a}+\oint_{\mathcal{B}_{1}} \delta A^{a} d S_{a} .
\end{aligned}
$$

If $\delta A^{a}$ were the variation of a quantity $A^{a}$, we could take the variation sign outside the $\mathcal{B}_{1}$ and $\mathcal{B}_{2}$ integrals and identify a boundary term $S_{\Sigma}$ for the spacelike or timelike segment. But $\delta A^{a}$ is not the variation of anything by itself, and these manipulations will not go through until we join segments together to form a closed hypersurface $\partial \mathscr{V}-$ see section $\amalg$ IID below.

\footnotetext{
${ }^{9}$ This is, of course, the usual boundary condition for Einstein's general relativity, i.e., the intrinsic geometry is held fixed on the (timelike and spacelike) boundary surfaces.
} 


\section{Null segment}

We next turn to the task of evaluating $\int \delta v^{\mu} d \Sigma_{\mu}$ on a null segment of $\partial \mathscr{V}$. We again denote this segment by $\Sigma$, and take it to be bounded in the past by a two-surface $\mathcal{B}_{1}$ and in the future by a two-surface $\mathcal{B}_{2}$.

\section{Preliminaries}

To handle the case of a null hypersurface we follow the methods reviewed in Sec. 3.1 of the Toolkit [28. We describe the hypersurface by the relation $\Phi\left(x^{\alpha}\right)=0$ for some scalar $\Phi$, with the convention that $\Phi$ increases toward the future. The hypersurface can also be described by the parametric equations $x^{\alpha}=x^{\alpha}\left(\lambda, \theta^{A}\right)$, where $\theta^{A}$ is constant on each null generator spanning the hypersurface, while $\lambda$ is a parameter on each generator. The null normal to the hypersurface is

$$
k_{\alpha}=-\mu \partial_{\alpha} \Phi,
$$

where $\mu$ is a (positive definite) scalar function on $\Sigma$; the minus sign ensures that $k^{\alpha}$ is a future-directed vector. The definition of the intrinsic coordinates $\left(\lambda, \theta^{A}\right)$ implies that the vectors

$$
k^{\alpha}=\frac{\partial x^{\alpha}}{\partial \lambda}, \quad e_{A}^{\alpha}=\frac{\partial x^{\alpha}}{\partial \theta^{A}}
$$

are tangent to the hypersurface $\oint^{10}$ and $k^{\alpha}=g^{\alpha \beta} k_{\beta}$ is orthogonal to the spacelike vectors $e_{A}^{\alpha}$. The null vector satisfies the geodesic equation

$$
k^{\beta} \nabla_{\beta} k^{\alpha}=\kappa k^{\alpha},
$$

with $\kappa\left(\lambda, \theta^{A}\right)$ measuring the failure of $\lambda$ to be an affine parameter on the null generators. The vector basis is completed with a second null vector $N^{\alpha}$, which is transverse to the hypersurface, orthogonal to $e_{A}^{\alpha}$, and which we choose to normalize by $k_{\alpha} N^{\alpha}=-1$. This allows us to write

$$
\kappa=-N_{\alpha} k^{\beta} \nabla_{\beta} k^{\alpha} .
$$

We let

$$
\gamma_{A B}:=g_{\alpha \beta} e_{A}^{\alpha} e_{B}^{\beta}
$$

be an induced metric on $\Sigma$, noting that a displacement on the hypersurface comes with the line element $d s^{2}=$ $\gamma_{A B} d \theta^{A} d \theta^{B}$. In this description, which exploits the congruence of null generators to construct a system of adapted intrinsic coordinates $\left(\lambda, \theta^{A}\right)$, the induced metric is not merely degenerate but explicitly two-dimensional. We let $\gamma^{A B}$ denote the matrix inverse to $\gamma_{A B}$, and $\gamma:=\operatorname{det}\left[\gamma_{A B}\right]$. We also introduce $e_{\alpha}^{A}:=\gamma^{A B} g_{\alpha \beta} e_{B}^{\beta}$.

The completeness relation for the inverse metric is given by

$$
g^{\alpha \beta}=-k^{\alpha} N^{\beta}-N^{\alpha} k^{\beta}+\gamma^{\alpha \beta}, \quad \gamma^{\alpha \beta}:=\gamma^{A B} e_{A}^{\alpha} e_{B}^{\beta} .
$$

According to Eq. (3.20) of the Toolkit, the directed surface element on $\Sigma$ is

$$
d \Sigma_{\alpha}=-k_{\alpha} \sqrt{\gamma} d^{2} \theta d \lambda
$$

with the convention that $d \Sigma_{\alpha} \propto \partial_{\alpha} \Phi$, with a positive factor of proportionality.

The two-tensor

$$
B_{A B}:=e_{A}^{\alpha} e_{B}^{\beta} \nabla_{\alpha} k_{\beta}
$$

governs the behavior of the congruence of null generators. It is typically decomposed as

$$
B_{A B}=\frac{1}{2} \Theta \gamma_{A B}+\sigma_{A B}
$$

${ }^{10}$ We emphasize that for a null boundary segment $\Sigma$, the normal $k_{\alpha}$ is orthogonal to the surface but $k^{\alpha}$ is tangent to it. Further, our convention is that $k^{\alpha}$ is future directed and hence $k_{\alpha}$ is past directed. 
with $\Theta:=\gamma^{A B} B_{A B}$ measuring the rate of expansion of the congruence, and $\sigma_{A B}$ its rate of shear. We have that

$$
\Theta=\frac{1}{\sqrt{\gamma}} \frac{\partial \sqrt{\gamma}}{\partial \lambda},
$$

which indicates that $\Theta$ is the relative rate of change of $\sqrt{\gamma} d^{2} \theta$, the cross-sectional area of a bundle of null generators.

We conclude these preliminary remarks with a discussion of the arbitrariness involved in the description of null hypersurfaces. First, the parametrization of the null generators is arbitrary, and this implies that $\lambda$ can be redefined independently on each generator, $\lambda \rightarrow \bar{\lambda}\left(\lambda, \theta^{A}\right)$. The tangent vector $k^{\alpha}$, therefore, is defined up to a multiplicative factor that can vary arbitrarily over the hypersurface. Second, the function $\Phi$ is itself arbitrary, and could be replaced by a different function that also vanishes on the hypersurface, $\Phi \rightarrow \bar{\Phi}(\Phi)$. For a given vector field $k_{\alpha}$ corresponding to a given choice of parametrization, the freedom to change $\Phi$ corresponds to the freedom to change $\mu$ by an arbitrary multiplicative factor in Eq. 2.32. Note that these two sources of arbitrariness are independent from one another: For a fixed choice of $\Phi$, a reparametrization changes $k^{\alpha}$ by an arbitrary multiplicative factor, which is then inherited by $\mu$ through Eq. 2.32); for a fixed choice of parametrization and $k^{\alpha}$, a change of $\Phi$ corresponds to a change of $\mu$ by an independent multiplicative factor.

\section{Variation of geometric quantities}

We next perform a variation $\delta g_{\alpha \beta}$ of the metric, and see how various geometric quantities defined on $\Sigma$ respond to the variation. It is again understood that the description of the hypersurface is unchanged during the variation, so that the equations $\Phi=0$ and $x^{\alpha}=x^{\alpha}\left(\lambda, \theta^{A}\right)$ keep their original form. This implies that the tangent vectors $k^{\alpha}$ and $e_{A}^{\alpha}$ are unaffected by the variation. We also assume that the hypersurface stays null during the variation, and therefore impose

$$
\delta\left(g_{\alpha \beta} k^{\alpha} k^{\beta}\right)=k^{\alpha} k^{\beta} \delta g_{\alpha \beta}=0
$$

in our manipulations. We further assume that the vectors $k^{\alpha}$ and $e_{A}^{\alpha}$ stay orthogonal during the variation, so that

$$
\delta\left(g_{\alpha \beta} e_{A}^{\alpha} k^{\beta}\right)=e_{A}^{\alpha} k^{\beta} \delta g_{\alpha \beta}=0
$$

At a later stage we shall impose the additional restriction that the variation of the induced two-metric $\gamma_{A B}:=g_{\alpha \beta} e_{A}^{\alpha} e_{B}^{\beta}$ should vanish, completing to six the count of fixed metric components on the hypersurface (the same count as for a timelike or spacelike boundary surface).

The statements that $\delta k^{\alpha}=0$ and $\delta e_{A}^{\alpha}=0$, together with the relations $k^{\alpha} k_{\alpha}=0$ and $e_{A}^{\alpha} k_{\alpha}=0$, imply that $k^{\alpha} \delta k_{\alpha}=0$ and $e_{A}^{\alpha} \delta k_{\alpha}=0$, which means that

$$
\delta k_{\alpha}=\delta a k_{\alpha}
$$

for some $\delta a$. There is actually a quantity $a$ whose variation is $\delta a$. To see this, recall the relation $k_{\alpha}=-\mu \nabla_{\alpha} \Phi$, which implies that $\delta k_{\alpha}=(\delta \ln \mu) k_{\alpha}$, so that

$$
a=\ln \mu \text {. }
$$

This quantity will play a very important role below. A similar calculation reveals that

$$
\delta e_{\alpha}^{A}=\delta a^{A} k_{\alpha}
$$

for some infinitesimal quantity $\delta a^{A}$.

Variation of $\nabla_{\alpha} k^{\mu}$ yields

$$
\delta\left(\nabla_{\alpha} k^{\mu}\right)=k^{\beta} \delta \Gamma_{\alpha \beta}^{\mu},
$$

and a short calculation also reveals that

$$
\delta\left(\nabla_{\alpha} k_{\beta}\right)=\left(\nabla_{\alpha} \delta a\right) k_{\beta}+\delta a \nabla_{\alpha} k_{\beta}-k_{\mu} \delta \Gamma_{\alpha \beta}^{\mu}
$$

For each one of these identities it is understood that $k^{\mu}$ or $k_{\alpha}$ is differentiated in the directions tangent to the hypersurface. 
Equations 2.34 and (2.47) immediately imply that

$$
\delta \kappa=-k^{\alpha} k^{\beta} N_{\mu} \delta \Gamma_{\alpha \beta}^{\mu} .
$$

Alternatively, we can write Eq. 2.34 in the form $k^{\alpha} \nabla_{\alpha} k_{\beta}=\kappa k_{\beta}$, and construct the variation using Eq. (2.48). This yields

$$
\delta \kappa=k^{\alpha} \nabla_{\alpha} \delta a+k^{\alpha} N^{\beta} k_{\mu} \delta \Gamma_{\alpha \beta}^{\mu} .
$$

We next examine the variation of $\Theta$, the rate of expansion of the congruence of null generators. In the first version of this calculation we write $\Theta=\gamma^{A B} B_{A B}$ and express the variation as $\delta \Theta=B_{A B} \delta \gamma^{A B}+\gamma^{A B} \delta B_{A B}$. Eqs. 2.39 and 2.48 imply that

$$
\delta B_{A B}=\delta a B_{A B}-e_{A}^{\alpha} e_{B}^{\beta} k_{\mu} \delta \Gamma_{\alpha \beta}^{\mu},
$$

and taking the trace returns

$$
\delta \Theta=B_{A B} \delta \gamma^{A B}+\Theta \delta a-\gamma^{\alpha \beta} k_{\mu} \delta \Gamma_{\alpha \beta}^{\mu} .
$$

In the second version of the calculation we write instead

$$
\Theta=\gamma_{\mu}^{\alpha} \nabla_{\alpha} k^{\mu}=e_{A}^{\alpha} e_{\mu}^{A} \nabla_{\alpha} k^{\mu}
$$

and take the variation using Eq. 2.47). We have

$$
\begin{aligned}
\delta \Theta & =e_{A}^{\alpha}\left(\delta e_{\alpha}^{A}\right) \nabla_{\alpha} k^{\mu}+e_{A}^{\alpha} e_{\mu}^{A} \delta\left(\nabla_{\alpha} k^{\mu}\right) \\
& =e_{A}^{\alpha}\left(\delta a^{A}\right) k_{\mu} \nabla_{\alpha} k^{\mu}+e_{A}^{\alpha} e_{\mu}^{A} k^{\beta} \delta \Gamma_{\alpha \beta}^{\mu} .
\end{aligned}
$$

The first term vanishes, and we end up with

$$
\delta \Theta=k^{\alpha} \gamma_{\mu}^{\beta} \delta \Gamma_{\alpha \beta}^{\mu}
$$

\section{Boundary term}

We may now evaluate $\int_{\Sigma} \delta v^{\mu} d \Sigma_{\mu}$ when $\Sigma$ is a null hypersurface. We recall Eq. 2.3 and write

$$
\delta v^{\mu} k_{\mu}=\left(g^{\alpha \beta} k_{\mu}-k^{\alpha} g_{\mu}^{\beta}\right) \delta \Gamma_{\alpha \beta}^{\mu},
$$

in which we insert the completeness relation (2.37). After some simple algebra we arrive at

$$
\delta v^{\mu} k_{\mu}=\left(k^{\alpha} k^{\beta} N_{\mu}-k^{\alpha} N^{\beta} k_{\mu}+\gamma^{\alpha \beta} k_{\mu}-k^{\alpha} \gamma_{\mu}^{\beta}\right) \delta \Gamma_{\alpha \beta}^{\mu} .
$$

We next use Eqs. 2.49, 2.50, 2.52, and 2.55 to replace each term involving $\delta \Gamma_{\alpha \beta}^{\mu}$ by variations of quantities defined on the hypersurface. We obtain

$$
\delta v^{\mu} k_{\mu}=k^{\alpha} \nabla_{\alpha} \delta a+\Theta \delta a-2 \delta(\kappa+\Theta)+B_{A B} \delta \gamma^{A B}
$$

With Eq. 2.41) this equation becomes

$$
\delta v^{\mu} k_{\mu}=\frac{1}{\sqrt{\gamma}} \frac{\partial}{\partial \lambda}(\sqrt{\gamma} \delta a)-2 \delta(\kappa+\Theta)+B_{A B} \delta \gamma^{A B}
$$

and this shall be our final expression for $\delta v^{\mu} k_{\mu}$.

We have found that the hypersurface integral is given by

$$
\int_{\Sigma} \delta v^{\mu} d \Sigma_{\mu}=\int_{\Sigma}\left[2 \delta(\kappa+\Theta)-B_{A B} \delta \gamma^{A B}\right] \sqrt{\gamma} d^{2} \theta d \lambda-\int_{\Sigma} \frac{\partial}{\partial \lambda}\left(\delta a \sqrt{\gamma} d^{2} \theta\right) d \lambda .
$$

Incorporating our assumption that $\Sigma$ is bounded in the future by a two-surface $\mathcal{B}_{2}$ and in the past by a two-surface $\mathcal{B}_{1}$, this is

$$
\int_{\Sigma} \delta v^{\mu} d \Sigma_{\mu}=\int_{\Sigma}\left[2 \delta(\kappa+\Theta)-B_{A B} \delta \gamma^{A B}\right] \sqrt{\gamma} d^{2} \theta d \lambda-\oint_{\mathcal{B}_{2}} \delta a \sqrt{\gamma} d^{2} \theta+\oint_{\mathcal{B}_{1}} \delta a \sqrt{\gamma} d^{2} \theta
$$


This can be expressed in a different form by manipulating the $\delta \Theta$ term. Because $\Theta=\partial_{\lambda} \ln \sqrt{\gamma}$ we have that

$$
\begin{aligned}
\int_{\Sigma} \delta \Theta \sqrt{\gamma} d^{2} \theta d \lambda & =\int_{\Sigma} \partial_{\lambda}(\delta \ln \sqrt{\gamma}) \sqrt{\gamma} d^{2} \theta d \lambda \\
& =-\int_{\Sigma}\left(\partial_{\lambda} \sqrt{\gamma}\right) \delta \ln \sqrt{\gamma} d^{2} \theta d \lambda+\oint_{\mathcal{B}_{2}} \sqrt{\gamma} \delta \ln \sqrt{\gamma} d^{2} \theta-\oint_{\mathcal{B}_{1}} \sqrt{\gamma} \delta \ln \sqrt{\gamma} d^{2} \theta \\
& =-\int_{\Sigma} \Theta \delta \sqrt{\gamma} d^{2} \theta d \lambda+\oint_{\mathcal{B}_{2}} \delta \sqrt{\gamma} d^{2} \theta-\oint_{\mathcal{B}_{1}} \delta \sqrt{\gamma} d^{2} \theta
\end{aligned}
$$

In the second and third terms the variation sign can be taken out of the integral, and in the first term we can write $\delta \sqrt{\gamma}=-\frac{1}{2} \sqrt{\gamma} \gamma_{A B} \delta \gamma^{A B}$. This yields

$$
\int_{\Sigma} \delta \Theta \sqrt{\gamma} d^{2} \theta d \lambda=\frac{1}{2} \int_{\Sigma} \Theta \gamma_{A B} \delta \gamma^{A B} \sqrt{\gamma} d^{2} \theta d \lambda+\delta \mathcal{A}_{2}-\delta \mathcal{A}_{1}
$$

where $\mathcal{A}_{j}:=\oint_{\mathcal{B}_{1}} \sqrt{\gamma} d^{2} \theta$ is the area of the two-surface $\mathcal{B}_{j}$. Substitution within Eq. 2.61] gives

$$
\int_{\Sigma} \delta v^{\mu} d \Sigma_{\mu}=\int_{\Sigma}\left[2 \delta \kappa-\left(B_{A B}-\Theta \gamma_{A B}\right) \delta \gamma^{A B}\right] \sqrt{\gamma} d^{2} \theta d \lambda+\delta \mathcal{A}_{2}-\oint_{\mathcal{B}_{2}} \delta a \sqrt{\gamma} d^{2} \theta-\delta \mathcal{A}_{1}+\oint_{\mathcal{B}_{1}} \delta a \sqrt{\gamma} d^{2} \theta .
$$

If we now assume that $\delta \gamma^{A B}=0$ on $\Sigma$ (as part of the variational conditions on the null surface - see the discussion above), the result simplifies to

$$
\begin{aligned}
\int_{\Sigma} \delta v^{\mu} d \Sigma_{\mu} & =2 \int_{\Sigma} \delta \kappa \sqrt{\gamma} d^{2} \theta d \lambda-\oint_{\mathcal{B}_{2}} \delta a \sqrt{\gamma} d^{2} \theta+\oint_{\mathcal{B}_{1}} \delta a \sqrt{\gamma} d^{2} \theta \\
& =\delta\left(2 \int_{\Sigma} \kappa \sqrt{\gamma} d^{2} \theta d \lambda-\oint_{\mathcal{B}_{2}} a \sqrt{\gamma} d^{2} \theta+\oint_{\mathcal{B}_{1}} a \sqrt{\gamma} d^{2} \theta\right) .
\end{aligned}
$$

This computation reveals the existence of a boundary term

$$
S_{\Sigma}=-2 \int_{\Sigma} \kappa \sqrt{\gamma} d^{2} \theta d \lambda+\oint_{\mathcal{B}_{2}} a \sqrt{\gamma} d^{2} \theta-\oint_{\mathcal{B}_{1}} a \sqrt{\gamma} d^{2} \theta
$$

for a segment $\Sigma$ of a null hypersurface. We note that by virtue of Eq. (2.41), the condition $\delta \gamma^{A B}=0$ automatically implies that $\delta \Theta=0$, and this term was therefore eliminated in Eq. (2.65). We note that our boundary term (2.66) differs from the one given in [6] by a term proportional to $\Theta$, since the $\delta \Theta$ term was retained there in their final expression for $\int_{\Sigma} \delta v^{\mu} d \Sigma_{\mu}$.

Our expression for $S_{\Sigma}$ pertains to an isolated segment of null hypersurface. This segment, however, is only part of a closed boundary $\partial \mathscr{V}$ of a finite domain $\mathscr{V}$ of spacetime. In particular, $\Sigma$ will be joined to other (spacelike, timelike, or null) segments comprising $\partial \mathscr{V}$ at $\mathcal{B}_{1}$ and $\mathcal{B}_{2}$, and hence we should expect additional contributions at these joints coming from the neighbouring segments. We will show below in Sec. IIF that with the addition of these contributions, $S_{\Sigma}$ becomes

$$
S_{\Sigma}(\text { joined })=-2 \int_{\Sigma} \kappa \sqrt{\gamma} d^{2} \theta d \lambda+2 \oint_{\mathcal{B}_{2}} a \sqrt{\gamma} d^{2} \theta-2 \oint_{\mathcal{B}_{1}} a \sqrt{\gamma} d^{2} \theta
$$

That is, the joint terms at $\mathcal{B}_{1}$ and $\mathcal{B}_{2}$ acquire a factor of two. Furthermore, the joining of segments forces $a$ to take the specific form $a=\ln |n \cdot k|+a_{0}$ when $\Sigma$ is joined to a spacelike or timelike surface with unit normal $n^{\alpha}$, or of the form $a=\ln (-k \cdot \bar{k})+a_{0}$ when it is joined to another null surface with normal $\bar{k}^{\alpha}$. Here, a dot indicates an inner product between vectors, for example $n \cdot k:=g_{\alpha \beta} n^{\alpha} k^{\beta}$, and $a_{0}$ is an arbitrary quantity that satisfies $\delta a_{0}=0$.

It is striking that the value of $S_{\Sigma}$ (joined) is ill-defined, first because it depends on the choice of parametrization for the null generators, and second because it depends on the choice of $a_{0}$. However, the variation of the boundary term is well-defined, because the parametrization and $a_{0}$ are fixed while taking the variation. For a stationary null hypersurface there exists a preferred parametrization $\lambda^{*}$ defined such that $\kappa, \gamma_{A B}$, and $a$ are all independent of $\lambda^{*}$. An example of this is a Killing horizon, for which $k^{\alpha}$ can be identified with the Killing vector $\xi^{\alpha}$ evaluated on the horizon, $\Phi$ is chosen to be equal to $\xi_{\alpha} \xi^{\alpha}$, and then $a=-\ln (2 \kappa)$. In this case $S_{\Sigma}$ (joined) reduces to

$$
S_{\Sigma}^{*}(\text { joined })=-2 \kappa^{*} \mathcal{A}\left(\lambda_{2}^{*}-\lambda_{1}^{*}\right),
$$

where $\mathcal{A}:=\int \sqrt{\gamma} d^{2} \theta$ is the cross-sectional area of the hypersurface. Note that the two joint terms in Eq. 2.67 have canceled here because the cross-sections are invariant under the Killing flow, i.e., $\mathcal{A}_{1}=\mathcal{A}_{2}=\mathcal{A}$ and $a_{1}=a_{2}$. 


\section{Reparametrizations}

It is instructive to work out what happens to $S_{\Sigma}$ (joined) when the parametrization of each generator is changed from $\lambda$ to $\bar{\lambda}=\bar{\lambda}\left(\lambda, \theta^{A}\right)$. The effect of this transformation on the various geometrical quantities was deduced in Ref. 29]. Defining $e^{-\beta}:=\partial \bar{\lambda} / \partial \lambda$, we have that

$$
\bar{k}^{\alpha}=e^{\beta} k^{\alpha}, \quad \bar{\gamma}_{A B}=\gamma_{A B}, \quad \bar{B}_{A B}=e^{\beta} B_{A B}, \quad \bar{\kappa}=e^{\beta}\left(\kappa+\partial_{\lambda} \beta\right) .
$$

The first relation implies that $\bar{a}=a+\beta$ (assuming that $\Phi$ is not changed during the reparametrization), and the third gives $\bar{\Theta}=e^{\beta} \Theta$. Inserting this within $S_{\Sigma}$ (joined) yields

$$
\begin{aligned}
\bar{S}_{\Sigma}(\text { joined }) & =S_{\Sigma}(\text { joined })-2 \int_{\Sigma} \partial_{\lambda} \beta \sqrt{\gamma} d^{2} \theta d \lambda+2 \oint_{\mathcal{B}_{2}} \beta \sqrt{\gamma} d^{2} \theta-2 \oint_{\mathcal{B}_{1}} \beta \sqrt{\gamma} d^{2} \theta \\
& =S_{\Sigma}(\text { joined })+2 \int_{\Sigma} \Theta \beta \sqrt{\gamma} d^{2} \theta d \lambda
\end{aligned}
$$

with the second expression following from the first after an integration by parts. As expected, in general the value of $S_{\Sigma}$ (joined) for a given spacetime is not invariant under a reparametrization of the null generators. An exception arises in the case of a stationary hypersurface, for which $\Theta=0$. In this case the boundary term is invariant under a reparametrization, and it will therefore return the same value as in Eq. (2.68) irrespective of the parameterization of the null generators (so long as the choice of $\Phi$ is fixed).

\section{Redefinition of $\Phi$}

We have pointed out that $S_{\Sigma}$ (joined) is ill-defined because it depends on the choice of parameter $\lambda$, and also because it depends on the choice of function $\Phi$ that describes the hypersurface. To conclude this discussion, we describe the change to $S_{\Sigma}$ (joined) that results when we perform the redefinition

$$
\Phi \rightarrow \bar{\Phi}(\Phi)
$$

assuming that $\bar{\Phi}=0$ when $\Phi=0$. We keep the parametrization fixed during this operation, so that $k_{\alpha}$ is unchanged as a vector field on the hypersurface. It is easy to see that under the redefinition $(2.71), k_{\alpha}$ is re-expressed as

$$
k_{\alpha}=-\bar{\mu} \partial_{\alpha} \bar{\Phi}, \quad \bar{\mu}:=\mu \frac{d \Phi}{d \bar{\Phi}} .
$$

This implies that $a:=\ln \mu$ is changed to

$$
\bar{a}=a+\ln \frac{d \Phi}{d \bar{\Phi}}
$$

this change is actually in the $a_{0}$ piece of $a$, since the remaining piece — given by $\ln |n \cdot k|$ or $\ln (-k \cdot \bar{k})$ - is fixed for a given parametrization. The boundary action becomes

$$
\bar{S}_{\Sigma}(\text { joined })=S_{\Sigma}(\text { joined })+2 \oint_{\mathcal{B}_{2}} \ln \frac{d \Phi}{d \bar{\Phi}} \sqrt{\gamma} d^{2} \theta-2 \oint_{\mathcal{B}_{2}} \ln \frac{d \Phi}{d \bar{\Phi}} \sqrt{\gamma} d^{2} \theta
$$

This shows that the value of $S_{\Sigma}$ (joined) for a given spacetime is not invariant under a redefinition of $\Phi$.

\section{Closed hypersurface: Timelike and spacelike segments}

In sections $\mathrm{IIB}$ and $\mathrm{IIC}$, we derived boundary terms for the gravitational action (1.2). However, as we noted there, our analysis only examined isolated (spacelike, timelike, or null) boundary segments, which are implicitly part of a closed boundary $\partial \mathscr{V}$ of a finite domain $\mathscr{V}$ of spacetime. As a result, we were unable to give a complete description of the boundary contributions arising at the joints between neighbouring segments. We repair this deficiency in the next sections by focusing on the intersection of various boundary segments.

We begin in this section by forming a closed hypersurface $\partial \mathscr{V}$ with a timelike segment $\mathcal{T}$ joined to two spacelike segments $\mathcal{S}_{1}$ (in the past) and $\mathcal{S}_{2}$ (in the future), as illustrated in Fig. 3. The intersection between $\mathcal{T}$ and $\mathcal{S}_{1}$ is 


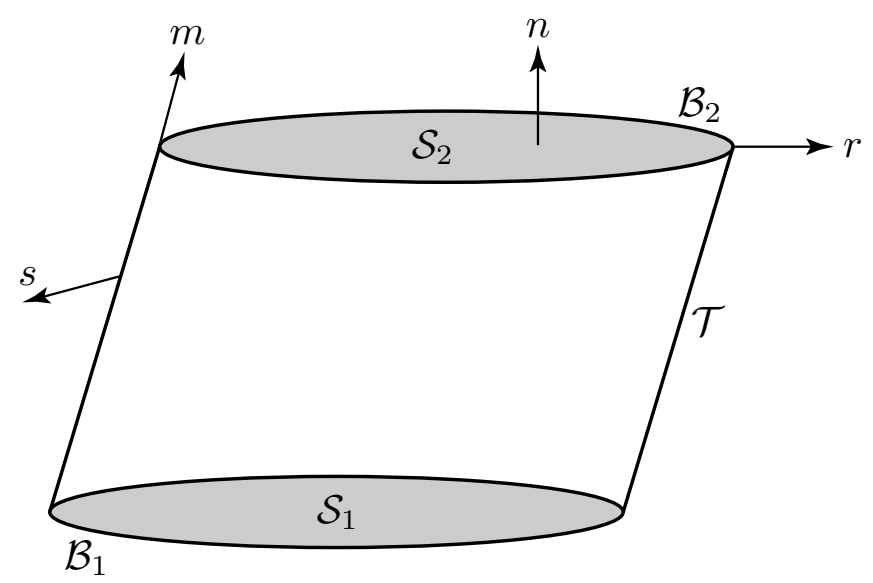

FIG. 3. Domain $\mathscr{V}$ bounded by a closed hypersurface $\partial \mathscr{V}$ consisting of a timelike segment $\mathcal{T}$ and two spacelike segments $\mathcal{S}_{1}$ and $\mathcal{S}_{2}$. The intersection between $\mathcal{T}$ and $\mathcal{S}_{j}$ is the closed two-surface $\mathcal{B}_{j}$.

the spacelike two-surface $\mathcal{B}_{1}$, and $\mathcal{B}_{2}$ is the intersection between $\mathcal{T}$ and $\mathcal{S}_{2}$. This is one of the cases that were first considered by Hayward [4].

To avoid confusion we must carefully specify the notation employed on each hypersurface segment and the joints between them. To set the stage we consider only $\mathcal{T}$ and the future surface $\mathcal{S} \equiv \mathcal{S}_{2}$, which intersect at $\mathcal{B} \equiv \mathcal{B}_{2}$; the past surface will be added at a later stage.

The spacelike hypersurface $\mathcal{S}$ has intrinsic coordinates $y^{a}$, a future-directed unit normal vector $n_{\alpha}$ with $\epsilon=n^{\alpha} n_{\alpha}=$ -1 , and a set of tangent vectors $e_{a}^{\alpha}=\partial x^{\alpha} / \partial y^{a}$. The induced metric is $h_{a b}$, the extrinsic curvature is $K_{a b}$, and the boundary quantity introduced in Eq. 2.17) is again denoted $\delta A^{a}$. The inverse metric on $\mathcal{S}$ is expressed as

$$
g^{\alpha \beta}=-n^{\alpha} n^{\beta}+h^{a b} e_{a}^{\alpha} e_{b}^{\beta} .
$$

The two-dimensional surface $\mathcal{B}$ can be given an embedding in $\mathcal{S}$. In this description, it has intrinsic coordinates $\theta^{A}$, an outward-directed unit normal vector $r_{a}$, and a set of tangent vectors $e_{A}^{a}=\partial y^{a} / \partial \theta^{A}$. The induced metric is $\gamma_{A B}$, and we have the completeness relation

$$
h^{a b}=r^{a} r^{b}+\gamma^{A B} e_{A}^{a} e_{B}^{b}
$$

We promote $r^{a}$ and $e_{A}^{a}$ to spacetime vectors according to $r^{\alpha}=r^{a} e_{a}^{\alpha}$ and $e_{A}^{\alpha}=e_{A}^{a} e_{a}^{\alpha}$, and combine the completeness relations to give

$$
g^{\alpha \beta}=-n^{\alpha} n^{\beta}+r^{\alpha} r^{\beta}+\gamma^{A B} e_{A}^{\alpha} e_{B}^{\beta},
$$

the inverse metric evaluated on $\mathcal{B}$.

Turning to the timelike hypersurface $\mathcal{T}$, we give it intrinsic coordinates $z^{j}$, an outward-directed unit normal vector $s_{\alpha}$ with $\epsilon=s^{\alpha} s_{\alpha}=+1$, and a set of tangent vectors $e_{j}^{\alpha}=\partial x^{\alpha} / \partial z^{j}$. The induced metric is $f_{j k}$, the extrinsic curvature is $L_{j k}$, and the boundary quantity introduced in Eq. 2.17] is now denoted $\delta B^{j}$. The inverse metric on $\mathcal{T}$ is

$$
g^{\alpha \beta}=s^{\alpha} s^{\beta}+f^{j k} e_{j}^{\alpha} e_{k}^{\alpha} .
$$

The two-dimensional surface $\mathcal{B}$ can also be given an embedding in $\mathcal{T}$. In this description, it has the same intrinsic coordinates $\theta^{A}$, but the outward-directed unit normal vector ${ }^{11}$ is now $m_{j}$, and the tangent vectors are $e_{A}^{j}=\partial z^{j} / \partial \theta^{A}$. The induced metric is $\gamma_{A B}$, and we have the completeness relation

$$
f^{j k}=-m^{j} m^{k}+\gamma^{A B} e_{A}^{j} e_{B}^{k}
$$

\footnotetext{
11 This normal $m^{j}$ is in the tangent space of $\mathcal{T}$ and "outward-directed" from this boundary surface. This also means that it is a futuredirected timelike unit vector.
} 
We promote $m^{j}$ and $e_{A}^{j}$ to spacetime vectors according to $m^{\alpha}=m^{j} e_{j}^{\alpha}$ and $e_{A}^{\alpha}=e_{A}^{j} e_{j}^{\alpha}$, and combine the completeness relations to give

$$
g^{\alpha \beta}=-m^{\alpha} m^{\beta}+s^{\alpha} s^{\beta}+\gamma^{A B} e_{A}^{\alpha} e_{B}^{\beta},
$$

an alternative expression for the inverse metric evaluated on $\mathcal{B}$.

Each pair $\left\{n^{\alpha}, r^{\alpha}\right\}$ and $\left\{m^{\alpha}, s^{\alpha}\right\}$ forms a set of (mutually orthogonal) unit normals on $\mathcal{B}$. Each pair can be used as a two-dimensional vector basis, and the bases are related by a spacetime boost. For example, we may write

$$
n^{\alpha}=\cosh \eta m^{\alpha}+\sinh \eta s^{\alpha}, \quad r^{\alpha}=\sinh \eta m^{\alpha}+\cosh \eta s^{\alpha}
$$

for some boost parameter $\eta$. A consequence of these relations is

$$
m^{\alpha}=\frac{1}{\cosh \eta} n^{\alpha}-\frac{\sinh \eta}{\cosh \eta} s^{\alpha}, \quad r^{\alpha}=\frac{\sinh \eta}{\cosh \eta} n^{\alpha}+\frac{1}{\cosh \eta} s^{\alpha},
$$

which expresses $m^{\alpha}$ (the normal to $\mathcal{B}$ embedded in $\mathcal{T}$ ) and $r^{\alpha}$ (the normal to $\mathcal{B}$ embedded in $\mathcal{S}$ ) in terms of $n^{\alpha}$ (the normal to $\mathcal{S}$ ) and $s^{\alpha}$ (the normal to $\mathcal{T}$ ).

Now from Eq. 2.31) we have that

$$
\int_{\mathcal{S}} \delta v^{\mu} d \Sigma_{\mu}=\delta\left(2 \int_{\mathcal{S}} K \sqrt{h} d^{3} y\right)-\oint_{\mathcal{B}} r_{a} \delta A^{a} \sqrt{\gamma} d^{2} \theta
$$

where we have inserted the relation $d S_{a}=r_{a} \sqrt{\gamma} d^{2} \theta$ for the surface element on $\mathcal{B}$. The same equation also produces

$$
\int_{\mathcal{T}} \delta v^{\mu} d \Sigma_{\mu}=\delta\left(-2 \int_{\mathcal{T}} L \sqrt{-f} d^{3} z\right)+\oint_{\mathcal{B}} m_{j} \delta B^{j} \sqrt{\gamma} d^{2} \theta
$$

where this time we used the relation $d S_{j}=-m_{j} \sqrt{\gamma} d^{2} \theta$ for the surface element. Following the notation introduced above, we are using $L$ to denote the trace of the extrinsic curvature on $\mathcal{T}$. Combining these two terms gives

$$
\int_{\mathcal{S}+\mathcal{T}} \delta v^{\mu} d \Sigma_{\mu}=\delta\left(2 \int_{\mathcal{S}} K \sqrt{h} d^{3} y-2 \int_{\mathcal{T}} L \sqrt{-f} d^{3} z\right)-\oint_{\mathcal{B}} \delta C \sqrt{\gamma} d^{2} \theta
$$

where $\delta C:=r_{a} \delta A^{a}-m_{j} \delta B^{j}$.

To evaluate the joint term on $\mathcal{B}$, we recall from Eq. (2.17) that $\delta A^{a}=+e_{\alpha}^{a} n_{\beta} \delta g^{\alpha \beta}$ and $\delta B^{j}=-e_{\alpha}^{j} s_{\beta} \delta g^{\alpha \beta}$. This gives

$$
\begin{aligned}
\delta C & =\left(r_{a} e_{\alpha}^{a} n_{\beta}+m_{j} e_{\alpha}^{j} s_{\beta}\right) \delta g^{\alpha \beta} \\
& =-\left(r^{a} e_{a}^{\alpha} n^{\beta}+m^{j} e_{j}^{\alpha} s^{\beta}\right) \delta g_{\alpha \beta} \\
& =-\left(r^{\alpha} n^{\beta}+m^{\alpha} s^{\beta}\right) \delta g_{\alpha \beta} \\
& =+\frac{\sinh \eta}{\cosh \eta}\left(-n^{\alpha} n^{\beta}+s^{\alpha} s^{\beta}\right) \delta g_{\alpha \beta}-\frac{1}{\cosh \eta}\left(n^{\alpha} s^{\beta}+s^{\alpha} n^{\beta}\right) \delta g_{\alpha \beta},
\end{aligned}
$$

where Eq. 2.82 was used in the last step.

On the other hand, we can vary the equation

$$
\sinh \eta=g^{\alpha \beta} n_{\alpha} s_{\beta}
$$

using Eq. 2.13 for $\delta n_{\alpha}$ and an analogous relation for $\delta s_{\beta}$. Simple algebra then returns

$$
\delta \eta=-\frac{\sinh \eta}{2 \cosh \eta}\left(-n_{\alpha} n_{\beta}+s_{\alpha} s_{\beta}\right) \delta g^{\alpha \beta}+\frac{1}{2 \cosh \eta}\left(n_{\alpha} s_{\beta}+s_{\alpha} n_{\beta}\right) \delta g^{\alpha \beta},
$$

and we conclude that $\delta C=2 \delta \eta$. Incorporating this in Eq. 2.85, we arrive at

$$
\int_{\mathcal{S}+\mathcal{T}} \delta v^{\mu} d \Sigma_{\mu}=\delta\left(2 \int_{\mathcal{S}} K \sqrt{h} d^{3} y-2 \int_{\mathcal{T}} L \sqrt{-f} d^{3} z-2 \oint_{\mathcal{B}} \eta \sqrt{\gamma} d^{2} \theta\right) .
$$

Hence the full boundary term for the hypersurface $\mathcal{S}+\mathcal{T}$ includes the Hayward term [4, proportional to the boost parameter $\eta$, at the joint $\mathcal{B}$. 
The joint term in Eq. 2.89 involves the parameter required to boost between the normal $n^{\alpha}$ to $\mathcal{T}$ and the normal $s^{\alpha}$ to $\mathcal{S}$, i.e., $\eta:=\operatorname{arcsinh}(n \cdot s)$, with $n \cdot s:=g_{\alpha \beta} n^{\alpha} s^{\beta}$. It is useful to give a simpler expression for $\eta$, one which will be adapted to other types of joints below. For this purpose, we introduce a basis of null vectors $k^{\alpha}$ and $\bar{k}^{\alpha}$; we take $k^{\alpha}$ and $\bar{k}^{\alpha}$ to be incoming and outgoing with respect to $\mathcal{T}$, respectively ${ }^{12}$, and we temporarily normalize them so that $k \cdot \bar{k}=-1$. In terms of this basis, we have

$$
n^{\alpha}=\frac{1}{2 A} k^{\alpha}+A \bar{k}^{\alpha}, \quad s^{\alpha}=-\frac{1}{2 B} k^{\alpha}+B \bar{k}^{\alpha},
$$

where $A:=-n \cdot k>0$ and $B:=-s \cdot k>0$. With these expressions, we can easily show that $\sinh \eta=\frac{1}{2}(A / B-B / A)$, so that

$$
\eta=\ln (-n \cdot k)-\ln (-s \cdot k) .
$$

Noting that $A=1 /(2 \bar{A})$ and $B=1 /(2 \bar{B})$ with $\bar{A}:=-n \cdot \bar{k}$ and $\bar{B}:=s \cdot \bar{k}, \eta$ can alternatively be expressed as

$$
\eta=-\ln (-n \cdot \bar{k})+\ln (s \cdot \bar{k}) .
$$

These expressions reveal that $\eta$ is independent of the normalization of the null vectors $k^{\alpha}$ and $\bar{k}^{\alpha}$, as it should be. The normalization condition $k \cdot \bar{k}=-1$, which facilitated the computations producing to Eqs. 2.91) and 2.92), can therefore be relaxed; the expressions are valid for arbitrarily normalized null vectors.

At this stage we introduce the past surface $\mathcal{S}_{1}$ and construct the closed hypersurface $\partial \mathscr{V}$. The previous analysis can again be applied to determine the boundary term on $\mathcal{S}_{1}$ and the joint $\mathcal{B}_{1}$ where the former intersects with $\mathcal{T}$. However, we must alter some signs to account for the fact that while $d \Sigma_{\mu}$ is outward-directed on all of the segments comprising $\partial \mathscr{V}$, we take $n^{\alpha}$ to be future-directed on both $\mathcal{S}_{1}$ and $\mathcal{S}_{2}$. The final result is

$$
\int_{\partial \mathscr{V}} \delta v^{\mu} d \Sigma_{\mu}=\delta\left(2 \int_{\mathcal{S}_{2}} K \sqrt{h} d^{3} y-2 \int_{\mathcal{T}} L \sqrt{-f} d^{3} z-2 \int_{\mathcal{S}_{1}} K \sqrt{h} d^{3} y-2 \oint_{\mathcal{B}_{2}} \eta \sqrt{\gamma} d^{2} \theta+2 \oint_{\mathcal{B}_{1}} \eta \sqrt{\gamma} d^{2} \theta\right) .
$$

Hence Eq. (2.4) implies

$$
S_{\partial \mathscr{V}}=-2 \int_{\mathcal{S}_{2}} K \sqrt{h} d^{3} y+2 \int_{\mathcal{T}} L \sqrt{-f} d^{3} z+2 \int_{\mathcal{S}_{1}} K \sqrt{h} d^{3} y+2 \oint_{\mathcal{B}_{2}} \eta \sqrt{\gamma} d^{2} \theta-2 \oint_{\mathcal{B}_{1}} \eta \sqrt{\gamma} d^{2} \theta
$$

and we have reproduced Hayward's expression for the complete boundary action when $\partial \mathscr{V}$ consists of the union of a timelike surface $\mathcal{T}$, a past spacelike surface $\mathcal{S}_{1}$, and a future spacelike surface $\mathcal{S}_{2}$. The boost parameter $\eta$ that appears in the integrals over $\mathcal{B}_{1}$ and $\mathcal{B}_{2}$ is defined by Eq. 2.87), and given more explicitly by Eqs. (2.91) and (2.92).

\section{E. Rules for timelike and spacelike joints}

The considerations of Sec. II D can easily be adapted to other types of joints between timelike and spacelike boundary segments. A number of relevant cases are illustrated in Fig. 4. a more complete set of situations was presented in Hayward's original work [4. For all these cases, the boost parameter $\eta$ can be expressed in terms of the projections of the normal vectors in the directions of the null vectors $k^{\alpha}$ and $\bar{k}^{\alpha}$ introduced previously.

In the situation depicted in panel $a$ of Fig. 4, we have a past boundary broken at the two-surface $\mathcal{B}$ into two spacelike segments of normal $n_{1}^{\alpha}$ and $n_{2}^{\alpha}$. In this case, the contribution from $\mathcal{B}$ to the boundary action is $-2 \oint_{\mathcal{B}} \eta_{a} d S$, where $d S:=\sqrt{\gamma} d^{2} \theta$ is a surface element on $\mathcal{B}$, and where the boost parameter is given by

$$
\eta_{a}=\ln \left(-n_{1} \cdot k\right)-\ln \left(-n_{2} \cdot k\right)=-\ln \left(-n_{1} \cdot \bar{k}\right)+\ln \left(-n_{2} \cdot \bar{k}\right) .
$$

In panel $b$, the past boundary is replaced by a future boundary, and the contribution to the boundary action is $2 \oint_{\mathcal{B}} \eta_{b} d S$ with $\eta_{b}=\eta_{a}$.

In the situation illustrated in panel $c$ of Fig. 4 , we have two timelike segments of normals $s_{1}^{\alpha}$ and $s_{2}^{\alpha}$ joined together at $\mathcal{B}$. In this case the contribution to the boundary action is $-2 \oint_{\mathcal{B}} \eta_{c} d S$, with

$$
\eta_{c}=\ln \left(-s_{1} \cdot k\right)-\ln \left(-s_{2} \cdot k\right)=-\ln \left(s_{1} \cdot \bar{k}\right)+\ln \left(s_{2} \cdot \bar{k}\right) .
$$

\footnotetext{
12 That is, both null vectors are future-directed, i.e., $k \cdot n<0$ and $\bar{k} \cdot n<0$, and then we choose $k \cdot s<0$ and $\bar{k} \cdot s>0$.
} 

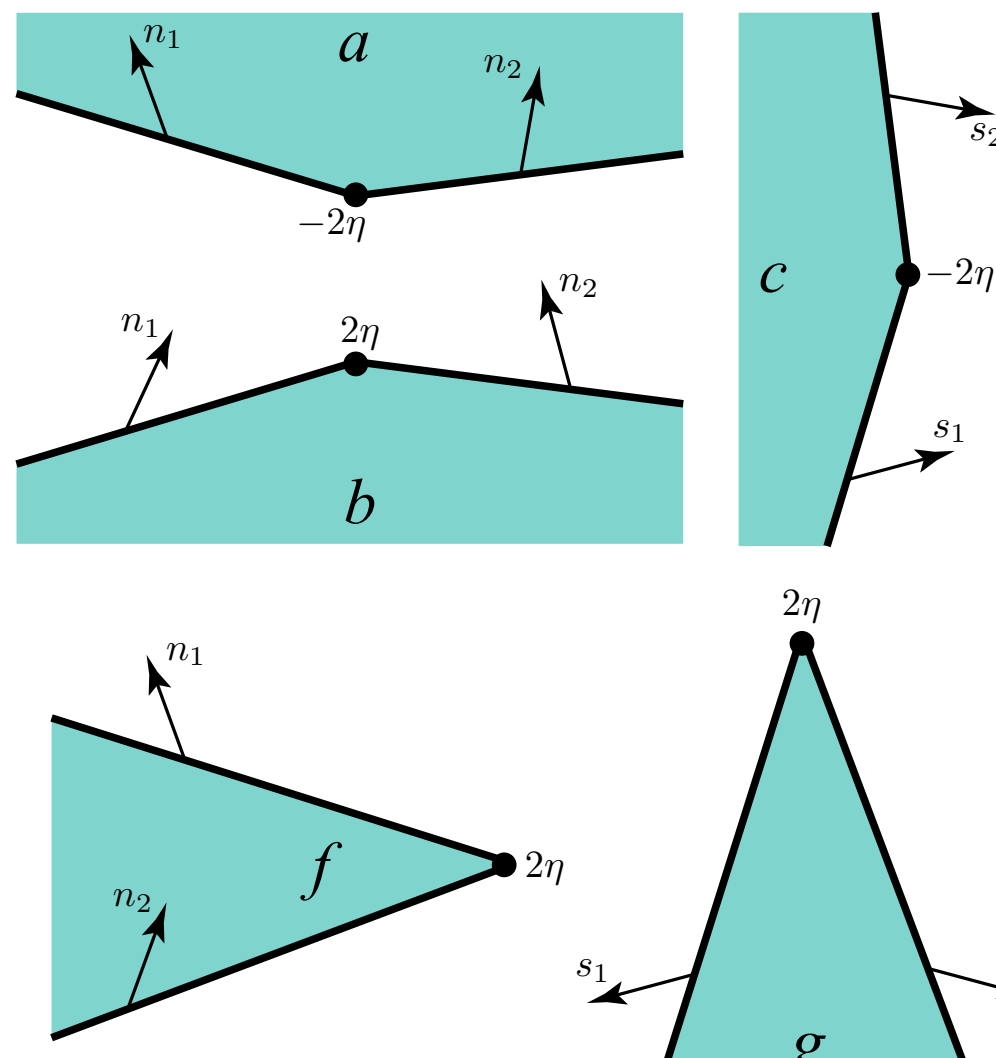
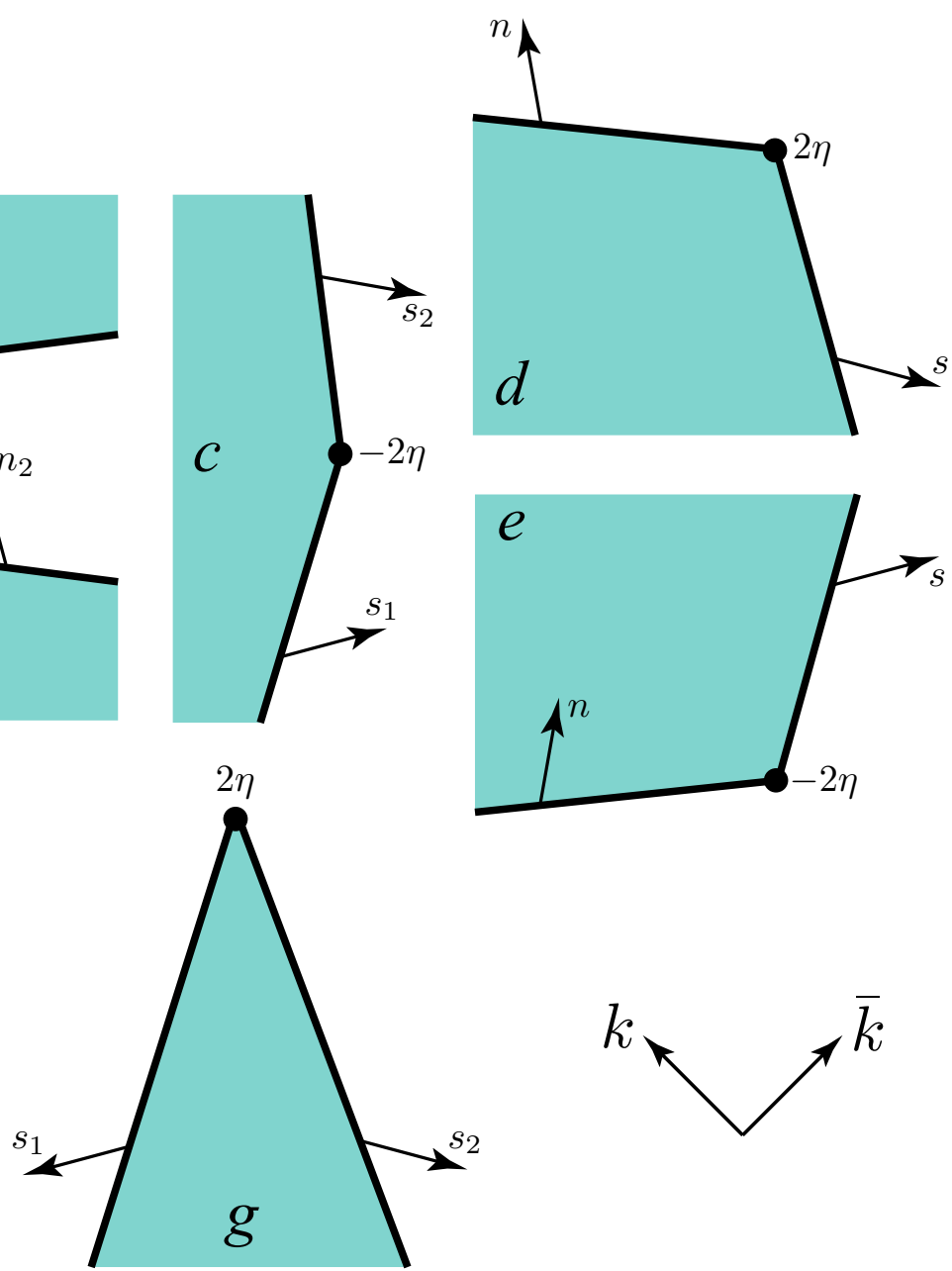

FIG. 4. Joint terms in the boundary action. In panel $a$, a past boundary is broken at $\mathcal{B}$ into two spacelike segments of normal $n_{1}^{\alpha}$ and $n_{2}^{\alpha}$; the contribution from $\mathcal{B}$ to the boundary action is $-2 \oint_{\mathcal{B}} \eta d S$. In panel $b$, a future boundary is broken into two spacelike segments; the contribution to the boundary action is $2 \oint_{\mathcal{B}} \eta d S$. In panel $c$, a timelike boundary is broken into two timelike segments of normal $s_{1}^{\alpha}$ and $s_{2}^{\alpha}$; the contribution to the boundary action is $-2 \oint_{\mathcal{B}} \eta d S$. In panel $d$, a timelike boundary of normal $s^{\alpha}$ is joined at $\mathcal{B}$ to a future, spacelike boundary of normal $n^{\alpha}$; the contribution to the boundary action is $2 \oint_{\mathcal{B}} \eta d S$. In panel $e$, a timelike boundary is joined to a past boundary, with contribution $-2 \oint_{\mathcal{B}} \eta d S$. In panel $f$, two spacelike boundaries are joined, with contribution $2 \oint_{\mathcal{B}} \eta d S$. Finally, two timelike boundaries are joined in panel $g$, with contribution $2 \oint_{\mathcal{B}} \eta d S$. In all panels the shaded region represents the interior of $\mathscr{V}$. The figure also shows the null vectors $k^{\alpha}$ and $\bar{k}^{\alpha}$, which are introduced in the main text.

Panel $d$ represents the situation examined in detail in Sec. IID which features a timelike boundary of normal $s^{\alpha}$ joined at $\mathcal{B}$ to a future, spacelike boundary of normal $n^{\alpha}$. In this case the contribution to the boundary action is $2 \oint_{\mathcal{B}} \eta_{d} d S$, with

$$
\eta_{d}=\ln (-n \cdot k)-\ln (-s \cdot k)=-\ln (-n \cdot \bar{k})+\ln (s \cdot \bar{k}) .
$$

In panel $e$, the future boundary is replaced by a past boundary, and the contribution to the boundary action becomes $2 \oint_{\mathcal{B}} \eta_{e} d S$, with $\eta_{e}=\eta_{d}$.

In the situation depicted in panel $f$, we have two spacelike segments of normal $n_{1}^{\alpha}$ and $n_{2}^{\alpha}$ joined together at $\mathcal{B}$. The joint gives a contribution $2 \oint_{\mathcal{B}} \eta_{f} d S$ to the boundary integral, with

$$
\eta_{f}=\ln \left(-n_{1} \cdot k\right)-\ln \left(-n_{2} \cdot k\right)=-\ln \left(-n_{1} \cdot \bar{k}\right)+\ln \left(-n_{2} \cdot \bar{k}\right) .
$$




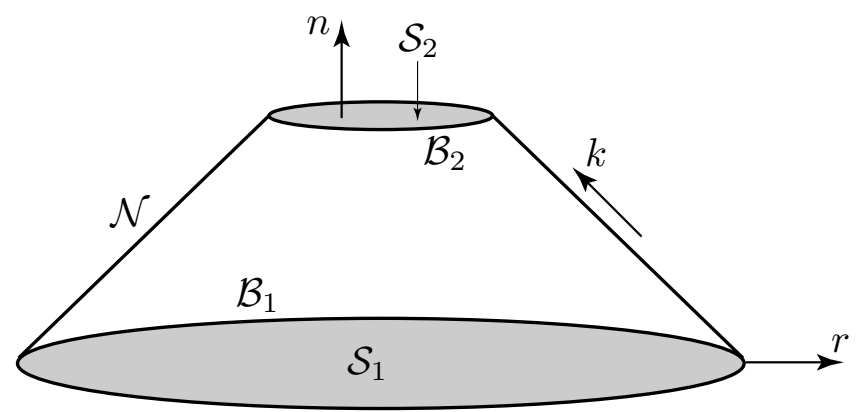

FIG. 5. A closed hypersurface $\partial \mathscr{V}$ consisting of a past spacelike surface $\mathcal{S}_{1}$, a truncated past null cone $\mathcal{N}$, and a future spacelike surface $\mathcal{S}_{2}$.

Finally, in panel $g$, we have two timelike segments of normal $s_{1}^{\alpha}$ and $s_{2}^{\alpha}$, and the joint contribution is $2 \oint_{\mathcal{B}} \eta_{g} d S$, with

$$
\eta_{g}=\ln \left(s_{1} \cdot k\right)-\ln \left(-s_{2} \cdot k\right)=-\ln \left(-s_{1} \cdot \bar{k}\right)+\ln \left(s_{2} \cdot \bar{k}\right) .
$$

A summary of the general rules for the construction of joint terms for intersections of spacelike and/or timelike boundary segments, as well as all of the other boundary terms in the gravitational action, appear in Appendix C. Note that our presentation of these joint terms differs somewhat from that originally given in [4, 25]; our results, however, are in precise agreement with those earlier works. Our construction also provides an explicit prescription for the sign of these terms, which was left ambiguous there.

\section{F. Closed hypersurface: Null and spacelike segments}

In this section, we form a closed hypersurface $\partial \mathscr{V}$ by combining null and spacelike hypersurfaces. Cases in which null segments are joined to timelike hypersurfaces can be treated along the same lines, but we shall not describe such a construction here. However, the appropriate joint terms in the gravitational action for these situations will be described in Sec. IIG.

\section{Past light cone truncated by spacelike segments}

We begin by joining a truncated past light cone $\mathcal{N}$ to two spacelike segments $\mathcal{S}_{1}$ (in the past) and $\mathcal{S}_{2}$ (in the future), as illustrated in Fig. 5. The intersection between $\mathcal{N}$ and $\mathcal{S}_{1}$ is the two-surface $\mathcal{B}_{1}$, and $\mathcal{B}_{2}$ is the intersection between $\mathcal{N}$ and $\mathcal{S}_{2}$. From Eq. 2.31, we have that

$$
\int_{\mathcal{S}_{2}} \delta v^{\mu} d \Sigma_{\mu}=\delta\left(2 \int_{\mathcal{S}_{2}} K \sqrt{h} d^{3} y\right)-\oint_{\mathcal{B}_{2}} r_{a} \delta A^{a} \sqrt{\gamma} d^{2} \theta
$$

where we inserted the relation $d S_{a}=r_{a} \sqrt{\gamma} d^{2} \theta$ for the surface element on $\mathcal{B}_{2}$. According to the conventions introduced in Sec. IIB $d \Sigma_{\mu} \propto \partial_{\mu} \Phi$, with the function $\Phi$ increasing toward the future of the hypersurface. Because this coincides with the direction out of $\mathscr{V}$, we have that $d \Sigma_{\mu}$ is correctly oriented on $\partial \mathscr{V}$. From Eq. 2.31) we also get

$$
\int_{\mathcal{S}_{1}} \delta v^{\mu} d \Sigma_{\mu}=\delta\left(-2 \int_{\mathcal{S}_{1}} K \sqrt{h} d^{3} y\right)+\oint_{\mathcal{B}_{1}} r_{a} \delta A^{a} \sqrt{\gamma} d^{2} \theta
$$

with the change in sign accounting for the fact that the outward direction now coincides with the past of $\mathcal{S}_{1}$. On the other hand, Eq. (2.65) gives

$$
\int_{\mathcal{N}} \delta v^{\mu} d \Sigma_{\mu}=\delta\left(2 \int_{\mathcal{N}} \kappa \sqrt{\gamma} d^{2} \theta d \lambda\right)-\oint_{\mathcal{B}_{2}} \delta a \sqrt{\gamma} d^{2} \theta+\oint_{\mathcal{B}_{1}} \delta a \sqrt{\gamma} d^{2} \theta
$$

with the same convention that $d \Sigma_{\mu} \propto \partial_{\mu} \Phi$, with $\Phi$ increasing toward the future of $\mathcal{N}$, which coincides with the exterior of $\mathscr{V}$. 


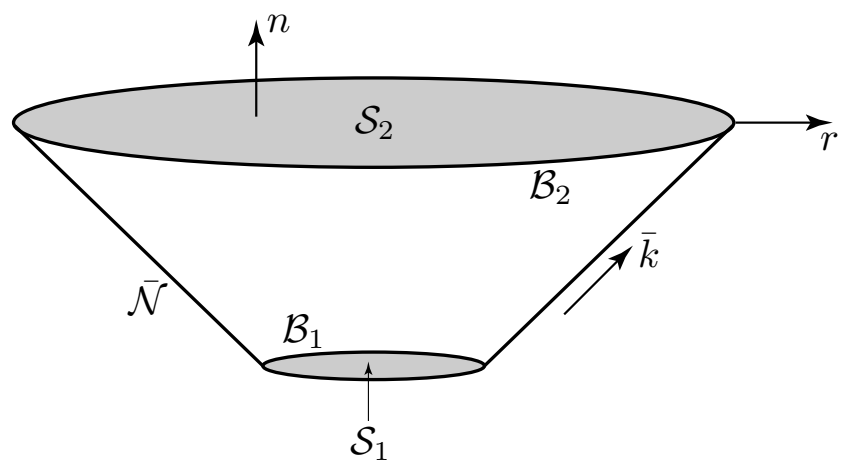

FIG. 6. A closed hypersurface $\partial \mathscr{V}$ consisting of a past spacelike surface $\mathcal{S}_{1}$, a truncated future null cone $\overline{\mathcal{N}}$, and a future spacelike surface $\mathcal{S}_{2}$.

Combining these expressions produces

$$
\begin{aligned}
\int_{\partial \mathscr{V}} \delta v^{\mu} d \Sigma_{\mu}= & \delta\left(2 \int_{\mathcal{S}_{2}} K \sqrt{h} d^{3} y+2 \int_{\mathcal{N}} \kappa \sqrt{\gamma} d^{2} \theta d \lambda-2 \int_{\mathcal{S}_{1}} K \sqrt{h} d^{3} y\right) \\
& -\oint_{\mathcal{B}_{2}}\left(\delta a+r_{a} \delta A^{a}\right) \sqrt{\gamma} d^{2} \theta+\oint_{\mathcal{B}_{1}}\left(\delta a+r_{a} \delta A^{a}\right) \sqrt{\gamma} d^{2} \theta .
\end{aligned}
$$

Below we shall show that $r_{a} \delta A^{a}=\delta a$ when a truncated past light cone is joined to a segment of spacelike hypersurface. This remarkable property allows us to write

$$
\int_{\partial \mathscr{V}} \delta v^{\mu} d \Sigma_{\mu}=\delta\left(2 \int_{\mathcal{S}_{2}} K \sqrt{h} d^{3} y+2 \int_{\mathcal{N}} \kappa \sqrt{\gamma} d^{2} \theta d \lambda-2 \int_{\mathcal{S}_{1}} K \sqrt{h} d^{3} y-2 \oint_{\mathcal{B}_{2}} a \sqrt{\gamma} d^{2} \theta+2 \oint_{\mathcal{B}_{1}} a \sqrt{\gamma} d^{2} \theta\right),
$$

and to identify the boundary action

$$
S_{\partial \mathscr{V}}=-2 \int_{\mathcal{S}_{2}} K \sqrt{h} d^{3} y-2 \int_{\mathcal{N}} \kappa \sqrt{\gamma} d^{2} \theta d \lambda+2 \int_{\mathcal{S}_{1}} K \sqrt{h} d^{3} y+2 \oint_{\mathcal{B}_{2}} a \sqrt{\gamma} d^{2} \theta-2 \oint_{\mathcal{B}_{1}} a \sqrt{\gamma} d^{2} \theta .
$$

We shall also show that in Eq. (2.105), $a$ must be of the form

$$
a=\ln (-n \cdot k)+a_{0},
$$

where $n^{\alpha}$ is the unit normal to $\mathcal{S}_{1}$ or $\mathcal{S}_{2}, k^{\alpha}$ is the null normal to $\mathcal{N}, n \cdot k$ is their inner product, and $a_{0}$ is an arbitrary quantity that satisfies $\delta a_{0}=0$.

Reiterating the statements made near the end of Sec. IIC 3, we observe that in general, the boundary action is ill-defined because it depends on the choices made for the parameter $\lambda$ and function $a_{0}$.

\section{Future light cone truncated by spacelike segments}

Next we form a closed hypersurface $\partial \mathscr{V}$ by joining a truncated future light cone $\overline{\mathcal{N}}$ to two spacelike segments $\mathcal{S}_{1}$ (in the past) and $\mathcal{S}_{2}$ (in the future); see Fig. 6. The intersection between $\mathcal{\mathcal { N }}$ and $\mathcal{S}_{1}$ is the two-surface $\mathcal{B}_{1}$, and $\mathcal{B}_{2}$ is the intersection between $\overline{\mathcal{N}}$ and $\mathcal{S}_{2}$.

The contributions to the $\partial \mathscr{V}$ integral coming from $\mathcal{S}_{2}$ and $\mathcal{S}_{1}$ are still given by Eqs. 2.100) and 2.101), respectively. The contribution from $\overline{\mathcal{N}}$, however, requires us to introduce an overall minus sign in Eq. $(2.102)$, so that

$$
\int_{\overline{\mathcal{N}}} \delta v^{\mu} d \Sigma_{\mu}=\delta\left(-2 \int_{\overline{\mathcal{N}}} \bar{\kappa} \sqrt{\gamma} d^{2} \theta d \bar{\lambda}\right)+\oint_{\mathcal{B}_{2}} \delta \bar{a} \sqrt{\gamma} d^{2} \theta-\oint_{\mathcal{B}_{1}} \delta \bar{a} \sqrt{\gamma} d^{2} \theta,
$$

where quantities with overbars refer to the null generators of the future light cone. The minus sign accounts for the fact that in the conventions employed to derive Eq. 2.65, $d \Sigma_{\mu} \propto \partial_{\mu} \Phi$ with $\Phi$ increasing toward the future of $\overline{\mathcal{N}}$. This direction corresponds to the interior of $\mathscr{V}$, and a correct outward orientation for $d \Sigma_{\mu}$ therefore requires the change of sign. 


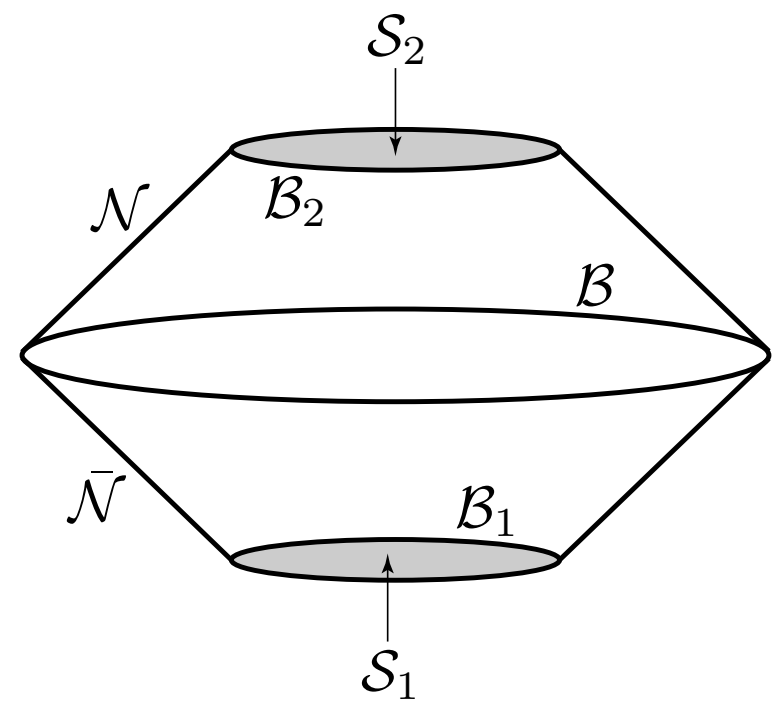

FIG. 7. A closed hypersurface $\partial \mathscr{V}$ consisting of a past spacelike surface $\mathcal{S}_{1}$, a truncated past null cone $\mathcal{N}$, a truncated future null cone $\overline{\mathcal{N}}$, and a future spacelike surface $\mathcal{S}_{2}$.

Combining these expressions produces

$$
\begin{aligned}
\int_{\partial \mathscr{V}} \delta v^{\mu} d \Sigma_{\mu}= & \delta\left(2 \int_{\mathcal{S}_{2}} K \sqrt{h} d^{3} y-2 \int_{\overline{\mathcal{N}}} \bar{\kappa} \sqrt{\gamma} d^{2} \theta d \bar{\lambda}-2 \int_{\mathcal{S}_{1}} K \sqrt{h} d^{3} y\right) \\
& +\oint_{\mathcal{B}_{2}}\left(\delta \bar{a}-r_{a} \delta A^{a}\right) \sqrt{\gamma} d^{2} \theta-\oint_{\mathcal{B}_{1}}\left(\delta \bar{a}-r_{a} \delta A^{a}\right) \sqrt{\gamma} d^{2} \theta
\end{aligned}
$$

Below we shall show that $r_{a} \delta A^{a}=-\delta \bar{a}$ when a truncated future light cone is joined to a segment of spacelike hypersurface. This allows us to write

$$
\int_{\partial \mathscr{V}} \delta v^{\mu} d \Sigma_{\mu}=\delta\left(2 \int_{\mathcal{S}_{2}} K \sqrt{h} d^{3} y-2 \int_{\overline{\mathcal{N}}} \bar{\kappa} \sqrt{\gamma} d^{2} \theta d \bar{\lambda}-2 \int_{\mathcal{S}_{1}} K \sqrt{h} d^{3} y+2 \oint_{\mathcal{B}_{2}} \bar{a} \sqrt{\gamma} d^{2} \theta-2 \oint_{\mathcal{B}_{1}} \bar{a} \sqrt{\gamma} d^{2} \theta\right),
$$

and to identify the boundary action

$$
S_{\partial \mathscr{V}}=-2 \int_{\mathcal{S}_{2}} K \sqrt{h} d^{3} y+2 \int_{\overline{\mathcal{N}}} \bar{\kappa} \sqrt{\gamma} d^{2} \theta d \bar{\lambda}+2 \int_{\mathcal{S}_{1}} K \sqrt{h} d^{3} y-2 \oint_{\mathcal{B}_{2}} \bar{a} \sqrt{\gamma} d^{2} \theta+2 \oint_{\mathcal{B}_{1}} \bar{a} \sqrt{\gamma} d^{2} \theta .
$$

Further, we shall show that in Eq. (2.110), $\bar{a}$ must be of the form

$$
\bar{a}=\ln (-n \cdot \bar{k})+\bar{a}_{0},
$$

where $n^{\alpha}$ is the unit normal to $\mathcal{S}_{1}$ or $\mathcal{S}_{2}, \bar{k}^{\alpha}$ is the null normal to $\overline{\mathcal{N}}, n \cdot \bar{k}$ is their inner product, and $\bar{a}_{0}$ is an arbitrary quantity that satisfies $\delta \bar{a}_{0}=0$.

Once more we observe that the boundary action is ill-defined because it depends on the choices made for the parameter $\bar{\lambda}$ and function $\bar{a}_{0}$.

\section{Past and future light cone truncated by spacelike segments}

As a final variation on the theme, we form $\partial \mathscr{V}$ by taking the union of a past spacelike surface $\mathcal{S}_{1}$, a truncated future null cone $\overline{\mathcal{N}}$, a truncated past null cone $\mathcal{N}$, and a future spacelike surface $\mathcal{S}_{2}$; see Fig. 7 . The intersection between $\overline{\mathcal{N}}$ and $\mathcal{S}_{1}$ is the two-surface $\mathcal{B}_{1}$, the intersection between $\overline{\mathcal{N}}$ and $\mathcal{N}$ is the two-surface $\mathcal{B}$, and $\mathcal{B}_{2}$ is the intersection between $\mathcal{N}$ and $\mathcal{S}_{2}$. 
With the contributions listed previously we have that

$$
\begin{aligned}
\int_{\partial \mathscr{V}} \delta v^{\mu} d \Sigma_{\mu}= & \delta\left(2 \int_{\mathcal{S}_{2}} K \sqrt{h} d^{3} y+2 \int_{\mathcal{N}} \kappa \sqrt{\gamma} d^{2} \theta d \lambda-2 \int_{\overline{\mathcal{N}}} \bar{\kappa} \sqrt{\gamma} d^{2} \theta d \bar{\lambda}-2 \int_{\mathcal{S}_{1}} K \sqrt{h} d^{3} y\right) \\
& -\oint_{\mathcal{B}_{2}}\left(\delta a+r_{a} \delta A^{a}\right) \sqrt{\gamma} d^{2} \theta+\oint_{\mathcal{B}}(\delta a+\delta \bar{a}) \sqrt{\gamma} d^{2} \theta-\oint_{\mathcal{B}_{1}}\left(\delta \bar{a}-r_{a} \delta A^{a}\right) \sqrt{\gamma} d^{2} \theta .
\end{aligned}
$$

We have already stated that $r_{a} \delta A^{a}=\delta a$ on $\mathcal{B}_{2}$ and $r_{a} \delta A^{a}=-\delta \bar{a}$ on $\mathcal{B}_{1}$. We may also show that $\delta \bar{a}=\delta a$ on $\mathcal{B}$, and use this property to simplify the expression. We note first that on $\mathcal{B}$, the null vectors $k^{\alpha}$ and $\bar{k}^{\alpha}$ satisfy

$$
k \cdot \bar{k}=-c,
$$

where $c$ is a positive scalar field. Next we take the variation of $c=-k_{\alpha} \bar{k}^{\alpha}$, recalling that $\delta \bar{k}^{\alpha}=0$ and invoking Eq. (2.44) for $\delta k_{\alpha}$; we find that $\delta c=c \delta a$. Doing the same with $c=-\bar{k}_{\alpha} k^{\alpha}$, we now find that $\delta c=c \delta \bar{a}$ and conclude that indeed, $\delta \bar{a}=\delta a$. Our manipulations also reveal that $\delta a=\delta \ln c=\delta \ln (-k \cdot \bar{k})$, so that $a=\ln (-k \cdot \bar{k})+\hat{a}_{0}$, where $\hat{a}_{0}$ is an arbitrary function such that $\delta \hat{a}_{0}=0$.

With all these results in hand, we find that

$$
\begin{aligned}
\int_{\partial \mathscr{V}} \delta v^{\mu} d \Sigma_{\mu}= & \delta\left(2 \int_{\mathcal{S}_{2}} K \sqrt{h} d^{3} y+2 \int_{\mathcal{N}} \kappa \sqrt{\gamma} d^{2} \theta d \lambda-2 \int_{\overline{\mathcal{N}}} \bar{\kappa} \sqrt{\gamma} d^{2} \theta d \bar{\lambda}-2 \int_{\mathcal{S}_{1}} K \sqrt{h} d^{3} y\right. \\
& \left.-2 \oint_{\mathcal{B}_{2}} a \sqrt{\gamma} d^{2} \theta+2 \oint_{\mathcal{B}} a \sqrt{\gamma} d^{2} \theta-2 \oint_{\mathcal{B}_{1}} \bar{a} \sqrt{\gamma} d^{2} \theta\right)
\end{aligned}
$$

and we have identified the boundary action

$$
\begin{aligned}
S_{\partial \mathscr{V}}= & -2 \int_{\mathcal{S}_{2}} K \sqrt{h} d^{3} y-2 \int_{\mathcal{N}} \kappa \sqrt{\gamma} d^{2} \theta d \lambda+2 \int_{\overline{\mathcal{N}}} \bar{\kappa} \sqrt{\gamma} d^{2} \theta d \bar{\lambda}+2 \int_{\mathcal{S}_{1}} K \sqrt{h} d^{3} y \\
& +2 \oint_{\mathcal{B}_{2}} a \sqrt{\gamma} d^{2} \theta-2 \oint_{\mathcal{B}} a \sqrt{\gamma} d^{2} \theta+2 \oint_{\mathcal{B}_{1}} \bar{a} \sqrt{\gamma} d^{2} \theta
\end{aligned}
$$

The joint terms all come with specific forms for the integrand: On $\mathcal{B}_{1}$ we have that $\bar{a}=\ln \left(-n_{1} \cdot \bar{k}\right)+\bar{a}_{0}$, where $n_{1}^{\alpha}$ is the unit normal to $\mathcal{S}_{1}$, on $\mathcal{B}_{2}$ we have that $a=\ln \left(-n_{2} \cdot k\right)+a_{0}$, where $n_{2}^{\alpha}$ is normal to $\mathcal{S}_{2}$, and on $\mathcal{B}$ we have instead $a=\ln (-k \cdot \bar{k})+\hat{a}_{0}$.

Again this boundary action 2.115 is ill-defined because it depends on the choices made for the parameters $\lambda$ and $\bar{\lambda}$, as well as the functions $a_{0}, \bar{a}_{0}$, and $\hat{a}_{0}$.

$$
\text { 4. Proof that } r_{a} \delta A^{a}= \pm \delta a \text { and } \delta a=\delta \ln (-n \cdot k)
$$

We now establish that

$$
r_{a} \delta A^{a}=-\zeta \delta a, \quad \delta a=\delta \ln (-n \cdot k)
$$

on a two-surface $\mathcal{B}$ formed from the intersection of a spacelike surface $\mathcal{S}$ and a null surface $\mathcal{N}$. Here, $\zeta=-1$ when $\mathcal{N}$ is a past light cone, and $\zeta=+1$ when it is a future light cone. The (future-directed) unit vector $n^{\alpha}$ is normal to $\mathcal{S}$, $k^{\alpha}$ is the (future-directed) normal to $\mathcal{N}$, and $n \cdot k:=g_{\alpha \beta} n^{\alpha} k^{\beta}$ is their inner product.

We rely on a system of adapted coordinates $x^{\alpha}=\left(\lambda, r, \theta^{A}\right)$ defined in an open domain $\mathscr{V}$ that includes $\mathcal{N}$ and $\mathcal{S}$. We have that $\lambda$ is a time coordinate, and surfaces of constant $\lambda$ provide a foliation of $\mathscr{V}$ in spacelike hypersurfaces; the coordinate is defined such that $\lambda=\lambda_{0}$ on $\mathcal{S}$. We also have that $r$ is constant on each member of a family of nested hypersurfaces, which can be either timelike or null; it is such that $r=r_{0}$ on $\mathcal{N}$. When intersected with a surface of constant $\lambda$ such as $\mathcal{S}$, the hypersurfaces of constant $r$ become nested spheres, and $\mathcal{B}$ is also described by $r=r_{0}$. Finally, the angular coordinates $\theta^{A}$ range over the spheres of constant $\lambda$ and $r$. The coordinates are illustrated in Fig. 8

In these coordinates, $\mathcal{N}$ is the hypersurface $r=r_{0}$, and $\left(\lambda, \theta^{A}\right)$ are intrinsic coordinates. When $\mathcal{N}$ is a past light cone $(\zeta=-1), r$ increases toward the future of $\mathcal{N}$, and when $\mathcal{N}$ is a future light cone $(\zeta=+1), r$ increases toward its past. The null generators are parametrized with $\lambda$, and the angular coordinates are calibrated to ensure that $\theta^{A}$ is constant on each generator. We have that $k^{\alpha}=(1,0,0,0), k_{\alpha}=(0, \zeta \alpha, 0,0)$ for some scalar $\alpha>0$, and $k_{\alpha}$ is orthogonal to $e_{A}^{\alpha}$. These relations imply that $g_{\lambda \lambda}=0, g_{\lambda r}=\zeta \alpha, g_{\lambda A}=0$, and $g_{A B}=\gamma_{A B}$ on $\mathcal{N}$. 


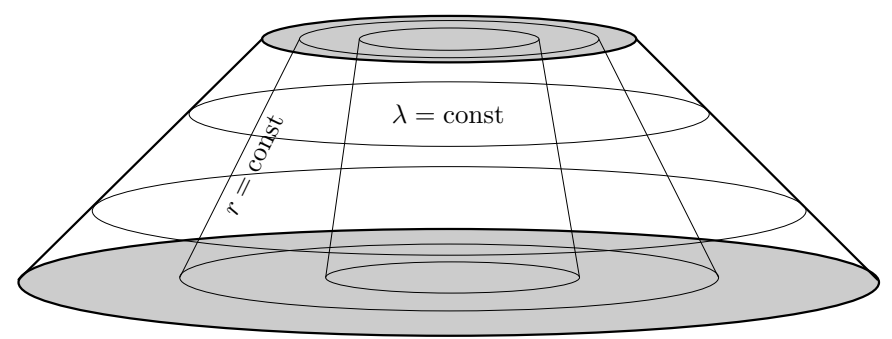

FIG. 8. Adapted coordinates $\left(\lambda, r, \theta^{A}\right)$ in $\mathscr{V}$.

The hypersurface $\mathcal{S}$ is described by $\lambda=$ const, and $\left(r, \theta^{A}\right)$ are intrinsic coordinates. Its unit normal is $n_{\alpha}=$ $(-1 / \beta, 0,0,0)$, with $\beta>0$ a scalar field on $\mathcal{S}$, and we have that $g^{\lambda \lambda}=-\beta^{2}$ on $\mathcal{S}$. The two-surface $\mathcal{B}$ is at $r=r_{0}$ in $\mathcal{S}$, and $\theta^{A}$ serve as intrinsic coordinates. Its unit normal is $r_{a}=(1 / \gamma, 0,0)$, where $\gamma>0$ is a scalar field on $\mathcal{B}$, and we have that $h^{r r}=\gamma^{2}$ on $\mathcal{B}$. Because $\mathcal{B}$ is also a surface $\lambda=$ const of $\Sigma$, its induced metric is necessarily $\gamma_{A B}$.

By virtue of the foregoing results, the spacetime metric evaluated on $\mathcal{B}$ has the structure

$$
g_{\alpha \beta}=\left(\begin{array}{cccc}
0 & \zeta \alpha & 0 & 0 \\
\zeta \alpha & h_{r r} & h_{r 2} & h_{r 3} \\
0 & h_{r 2} & \gamma_{22} & \gamma_{23} \\
0 & h_{r 3} & \gamma_{23} & \gamma_{33}
\end{array}\right),
$$

and the spatial metric $h_{a b}$ is given by the submatrix that excludes the first row and column. A key observation is that with $h_{a b}$ fixed on $\mathcal{S}$ and $\gamma_{A B}$ fixed on $\mathcal{N}$, the only variable component of the metric is $g_{\lambda r}=\zeta \alpha$. Calculation of $g^{\alpha \beta}$ and $h^{a b}$ reveals that

$$
\beta \gamma=\alpha^{-1}
$$

a result that will be required presently.

We may now proceed with the derivation of Eq. (2.116). We first invoke Eq. (2.17) and calculate

$$
r_{a} \delta A^{a}=r_{a} e_{\alpha}^{a} n_{\beta} \delta g^{\alpha \beta}=-r^{a} e_{a}^{\alpha} n^{\beta} \delta g_{\alpha \beta}=-r^{r} n^{\lambda} \delta g_{\lambda r} .
$$

Writing $r^{r}=h^{r a} r_{a}=h^{r r} / \gamma=\gamma, n^{\lambda}=g^{\lambda \alpha} n_{\alpha}=-g^{\lambda \lambda} / \beta=\beta$, we may conclude that

$$
r_{a} \delta A^{a}=-\zeta \beta \gamma \delta \alpha=-\zeta \frac{\delta \alpha}{\alpha}=-\zeta \delta \ln \alpha .
$$

The definition of $a:=\ln \mu$ is provided by the equation $k_{\alpha}=\zeta \mu \partial_{\alpha} \Phi$, which relates $k_{\alpha}$ to the gradient of an arbitrary function $\Phi$ that goes to zero on $\mathcal{N}$. In our adapted coordinates we can always write $\Phi=\left(r-r_{0}\right) \Psi\left(\lambda, r, \theta^{A}\right)$, where $\Psi$ is another arbitrary function, and conclude that $k_{r}=\zeta \mu \Psi$. Since this must be equal to $\zeta \alpha$, we have that $\alpha=\mu \Psi$, or $a=\ln \alpha-\ln \Psi$. Because $\Phi$ and $\Psi$ are fixed during the variation, we have that $\delta a=\delta \ln \alpha$, and

$$
r_{a} \delta A^{a}=-\zeta \delta a .
$$

The first part of Eq. (2.116) is thus established. To establish the second part we observe that $n \cdot k=k^{\alpha} n_{\alpha}=-1 / \beta=$ $-\gamma \alpha$, so that $\ln (-n \cdot k)=\ln \gamma+\ln \alpha$. But $\gamma^{2}=h^{r r}$ is fixed during the variation, so

$$
\delta a=\delta \ln \alpha=\delta \ln (-n \cdot k),
$$

as required. Notice that $\delta a$ is now expressed independently of the adapted coordinates. This relation can be integrated to yield

$$
a=\ln (-n \cdot k)+a_{0},
$$

where $a_{0}$ is an arbitrary scalar field on $\mathcal{B}$ whose variation $\delta a_{0}$ is required to vanish.

The lesson behind the result of Eq. 2.123) is that while the piece $\ln (-n \cdot k)$ of $a$ becomes determined when a segment of null hypersurface is joined to a spacelike segment, the remaining piece $a_{0}$ continues to be arbitrary. The first piece $\ln (-n \cdot k)$ contains the dependence on the choice of parametrization, while the second piece $a_{0}$ contains the dependence on the choice of $\Phi$. 

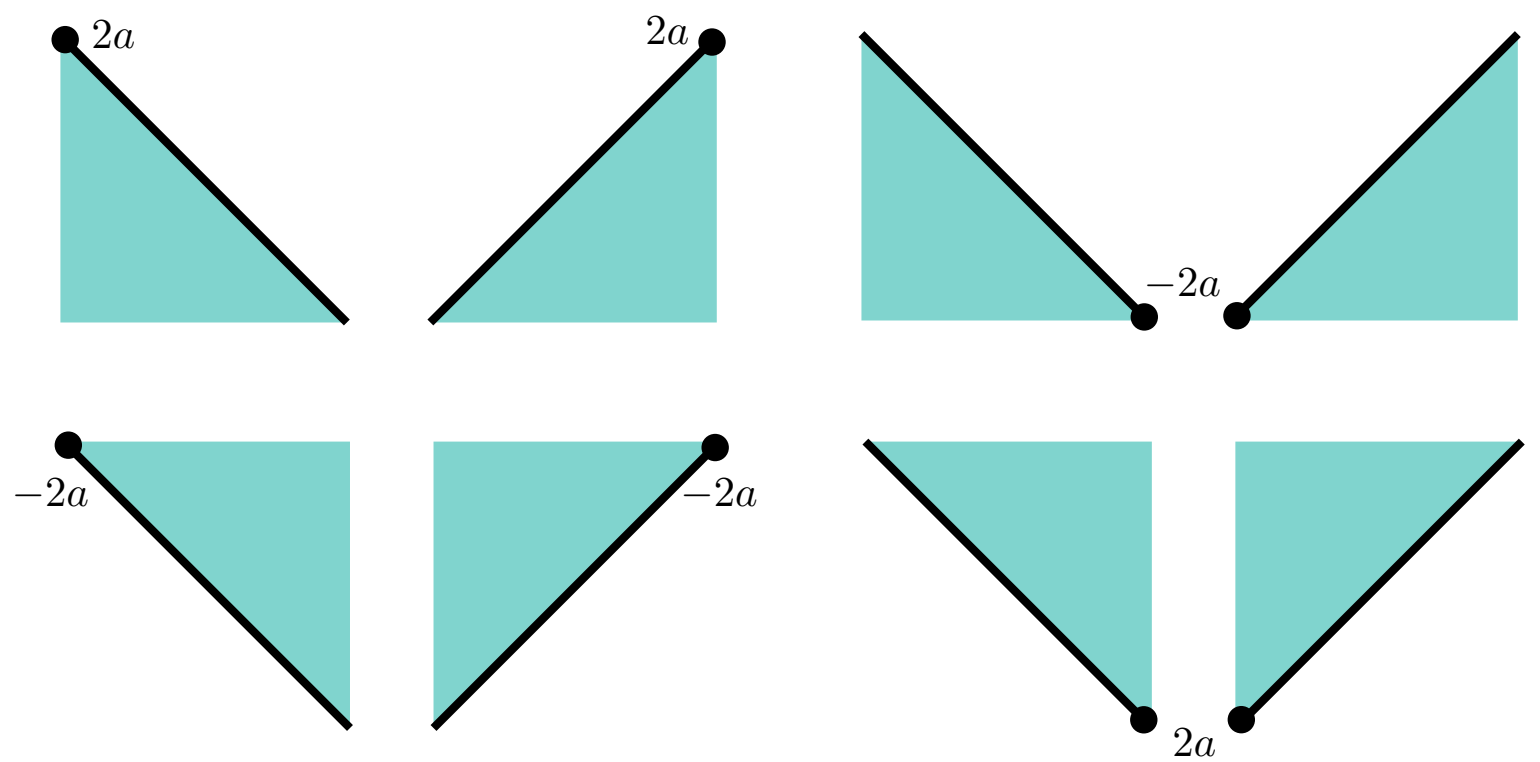

FIG. 9. Joint terms in the boundary action. The upper-left panel displays a null segment joined at $\mathcal{B}$ to another segment (not shown) which can be either spacelike, timelike, or null; because $\mathcal{B}$ is a future boundary to the null segment and the outward direction is a future direction, the contribution from $\mathcal{B}$ to the boundary action is $2 \oint_{\mathcal{B}} a d S$. In the upper-right panel, $\mathcal{B}$ is a past boundary, the outward direction continues to be a future direction, and the contribution to the boundary action is $-2 \oint_{\mathcal{B}} a d S$. In the lower-left panel, $\mathcal{B}$ is again a future boundary, but the outward direction is now a past direction; for these cases the contribution to the boundary action is $-2 \oint_{\mathcal{B}} a d S$. In the lower-right panel, $\mathcal{B}$ is a past boundary, the outward direction is a past direction, and the contribution to the boundary action is $2 \oint_{\mathcal{B}} a d S$. In all panels the shaded region indicates the interior of $\mathscr{V}$.

\section{G. Rules for null joints}

The considerations of Sec. IIF can easily be adapted to other types of joints involving one or two null hypersurfaces. All possible cases are illustrated in Fig. 9, which displays a null segment and its boundary $\mathcal{B}$; the (null, timelike, or spacelike) segment to which it is joined is not shown. When $\mathcal{B}$ is a future boundary and the outward direction across the null hypersurface coincides with the future direction (upper-left panel), the contribution to the boundary action is $2 \oint_{\mathcal{B}} a d S$, where $d S:=\sqrt{\gamma} d^{2} \theta$ is the surface element on $\mathcal{B}$. When $\mathcal{B}$ is a past boundary and the outward direction still coincides with the future direction (upper-right panel), the contribution to the boundary action is $-2 \oint_{\mathcal{B}}$ adS. When $\mathcal{B}$ is a future boundary and the outward direction coincides with the past direction (lower-left panel), the contribution to the boundary action is again $-2 \oint_{\mathcal{B}} a d S$. And finally, when $\mathcal{B}$ is a past boundary and the outward direction coincides with the past direction (lower-right panel), the contribution to the boundary action is $2 \oint_{\mathcal{B}} a d S$.

When a null segment is joined at $\mathcal{B}$ to a spacelike segment, we have seen that

$$
a^{\text {spacelike }}=\ln (-n \cdot k)+a_{0}^{\text {spacelike }},
$$

where $n^{\alpha}$ is the unit normal to the spacelike segment, $k^{\alpha}$ is the null normal, $n \cdot k:=g_{\alpha \beta} n^{\alpha} k^{\beta}$ is their inner product, and $a_{0}^{\text {spacelike }}$ is an arbitrary scalar field on $\mathcal{B}$ required to have a vanishing variation. When the null segment is joined instead to a timelike segment, a calculation similar to the one carried out in Sec. IIF 4 would reveal that in this case,

$$
a^{\text {timelike }}=\ln |s \cdot k|+a_{0}^{\text {timelike }},
$$

where $s^{\alpha}$ is the unit outward normal to the timelike segment, and $a_{0}^{\text {timelike }}$ in another arbitrary scalar field with zero variation. And when the null segment is joined to another null segment, we have seen that

$$
a^{\text {null }}=\ln (-k \cdot \bar{k})+a_{0}^{\text {null }},
$$

where $\bar{k}^{\alpha}$ is the normal to the second null segment, and $a_{0}^{\text {null }}$ is yet another arbitrary scalar field with vanishing variation. 


\section{H. Additivity rules}

We will say that an action is additive if the action for the union of two regions $\mathscr{V}_{1}$ and $\mathscr{V}_{2}$ is equal to the sum of the actions for $\mathscr{V}_{1}$ and $\mathscr{V}_{2}$ separately, when the action is evaluated on field configurations that extend across $\mathscr{V}=\mathscr{V}_{1} \cup \mathscr{V}_{2}$. This is not a property that is typically discussed in the context of classical field theory, but it was in fact a primary consideration in [2]. There, addivity of the gravitational action was argued to be a requirement for quantum amplitudes (as described by path integrals in quantum gravity) to be additive, and this was presented as a motivation to introduce the Gibbons-Hawking-York boundary term. However, it was subsequently shown that when taking into account the contribution of joint terms, the gravitational action $S=S_{\mathscr{V}}+\mathcal{S}_{\partial \mathscr{V}}$ is not additive in general [25]. More precisely, obstacles to additivity arise from timelike joints (at the intersection of two timelike boundary surfaces). The volume and hypersurface terms are all properly additive, and there is no obstacle to additivity coming from joints (between timelike and/or spacelike segments) that are entirely spacelike — the case considered throughout this paper.

The consideration of null boundary segments creates additional obstacles to additivity, due to the arbitrariness associated with joint terms. An example of this situation is provided by Fig. 7, which can be viewed as the union of Figs. 5 and 6, with the $\mathcal{S}_{1}$ of Fig. 5 identified with the $\mathcal{S}_{2}$ of Fig. 6. In this case we find that according to Eq. 2.115, the joint term coming from $\mathcal{B}_{1} \equiv \mathcal{B}_{2} \equiv \mathcal{B}$ is given by

$$
S_{\mathcal{B}}[\text { Fig. } 7]=-2 \oint_{\mathcal{B}} a \sqrt{\gamma} d^{2} \theta,
$$

with $a=\ln (-k \cdot \bar{k})+\hat{a}_{0}$. On the other hand, Eqs. 2.105 and 2.110 imply

$$
S_{\mathcal{B}}[\text { Fig. 5] Fig. } 6]=-2 \oint_{\mathcal{B}}\left(a_{1}+\bar{a}_{2}\right) \sqrt{\gamma} d^{2} \theta,
$$

with $a_{1}=\ln \left(-n_{1} \cdot k\right)+a_{01}$ and $\bar{a}_{2}=\ln \left(-n_{2} \cdot \bar{k}\right)+\bar{a}_{02}$; because the $\mathcal{S}_{1}$ of Fig. 5 is identified with the $\mathcal{S}_{2}$ of Fig. 6 . we have that $n_{1}^{\alpha} \equiv n_{2}^{\alpha} \equiv n^{\alpha}$. To work out the relation between $a$ and $a_{1}+\bar{a}_{2}$, we decompose the null vectors $k^{\alpha}$ and $\bar{k}^{\alpha}$ in a basis consisting of the mutually orthogonal unit vectors $n^{\alpha}$ and $r^{\alpha}$, the second vector pointing out of $\mathcal{B}$. We have $k^{\alpha}=A\left(n^{\alpha}-r^{\alpha}\right), \bar{k}^{\alpha}=\bar{A}\left(n^{\alpha}+r^{\alpha}\right)$ for some scalars $A$ and $\bar{A}$, and it follows that $n \cdot k=-A, n \cdot \bar{k}=-\bar{A}$, and $k \cdot \bar{k}=-2 A \bar{A}$. We next find that

$$
a-\left(a_{1}+\bar{a}_{2}\right)=\ln 2+\hat{a}_{0}-\left(a_{01}+\bar{a}_{02}\right)
$$

and observe that the two versions of $S_{\mathcal{B}}$ disagree unless the right-hand side happens to vanish. Failure to achieve this would result in a gravitational action that is not properly additive.

It is possible to exploit the arbitrariness of $\hat{a}_{0}, a_{01}$, and $\bar{a}_{02}$ to produce a gravitational action which is additive. That is, we demand additivity for the boundary terms at spacelike joints arising when null segments intersect other boundary segments. This requirement, in fact, becomes a prescription to remove the arbitrariness of these joint terms. For example, in Eq. 2.129 , the simplest way to achieve addivity is to set $a_{01}=\bar{a}_{02}=0$ and $\hat{a}_{0}=-\ln 2$. These choices give us additivity rules for null joints, which can be formulated as follows:

- spacelike rule: for a joint between null and spacelike hypersurfaces, assign $a=\ln (-n \cdot k)$, where $k^{\alpha}$ is the future-directed normal to the null hypersurface (with arbitrary normalization), and $n^{\alpha}$ is the future-directed unit normal to the spacelike hypersurface;

- timelike rule: for a joint between null and timelike hypersurfaces, assign $a=\ln |s \cdot k|$, where $s^{\alpha}$ is the outward-directed unit normal to the timelike hypersurface;

- null rule: for a joint between two null hypersurfaces, assign $a=\ln \left(-\frac{1}{2} k \cdot \bar{k}\right)$, where $k^{\alpha}$ is the future-directed normal to the first null hypersurface (with arbitrary normalization), and $\bar{k}^{\alpha}$ is the future-directed normal to the second hypersurface (also with arbitrary normalization).

The additivity rules eliminate the arbitrariness of the joint terms, once a choice of normalization has been made for the null normals.

We may test the applicability of these rules in a few examples. In Fig. 10 we show the first few examples of intersections between timelike and/or spacelike boundary segments considered in Fig. 4, but with a null surface now extending from each joint to subdivide the spacetime region into two parts. Combining the joint rules in section II E with those above for joints involving null segments, we see in each case that addivity is indeed satisfied:

In panel $a$ of Fig. 10 , the composite figure gives rise to a joint term $-2 \eta$ with $\eta=-\ln \left(-n_{1} \cdot \bar{k}\right)+\ln \left(-n_{2} \cdot \bar{k}\right)$ from Eq. 2.95. On the other hand, the null joint on the left contributes $2 a_{1}$, while the one on the right contributes $-2 a_{2}$. 

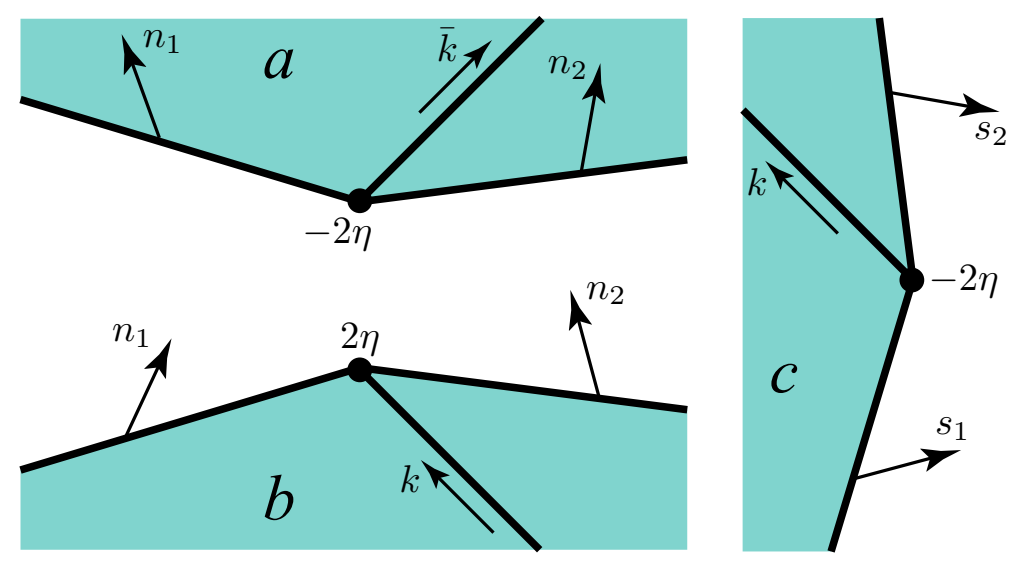

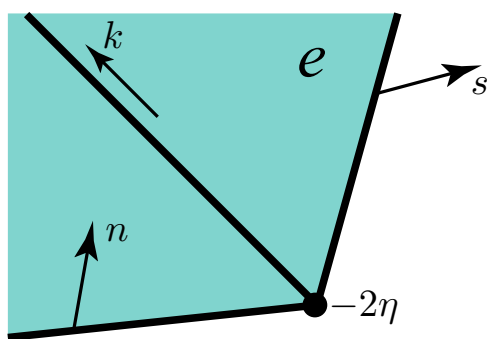

FIG. 10. Composition of boundary actions. In panels $a$ and $b$, a spacelike/spacelike joint is obtained by taking the union of two null/spacelike joints. In panel $c$, a timelike/timelike joint is the union of two null/timelike joints. In panels $d$ and $e$, a spacelike/timelike joint is the union of a null/spacelike joint and a null/timelike joint.

The spacelike rule makes the assignments $a_{1}=\ln \left(-n_{1} \cdot \bar{k}\right)$ and $a_{2}=\ln \left(-n_{2} \cdot \bar{k}\right)$, and we recover $-\eta=a_{1}-a_{2}$, as required for the proper additivity of the gravitational action. In panel $b$, the spacelike/spacelike joint contributes $2 \eta$ with $\eta=\ln \left(-n_{1} \cdot k\right)-\ln \left(-n_{2} \cdot k\right)$, again from Eq. (2.95). The null joint on the left gives $2 a_{1}$ with $a_{1}=\ln \left(-n_{1} \cdot k\right)$, and the one on the right gives $-2 a_{2}$ with $a_{2}=\ln \left(-n_{2} \cdot k\right)$. We have that $\eta=a_{1}-a_{2}$, and once again the boundary action is additive.

In panel $c$, we have that the timelike/timelike joint contributes a boundary term $-2 \eta$ with $\eta=\ln \left(-s_{1} \cdot k\right)-\ln \left(-s_{2} \cdot k\right)$ from Eq. (2.96). The null joint on the bottom contributes $-2 a_{1}$, while the one on the top gives $2 a_{2}$. The timelike rule makes the assignments $a_{1}=\ln \left(-s_{1} \cdot k\right)$ and $a_{2}=\ln \left(-s_{2} \cdot k\right)$, and we find that $-\eta=-a_{1}+a_{2}$, as required by additivity. In panel $d$, the composite figure comes with a contribution $2 \eta$ from the joint, with $\eta=-\ln (-n \cdot \bar{k})+\ln (s \cdot \bar{k})$ from Eq. (2.97). The null joint on the top contributes $-2 a_{1}$ with $a_{1}=\ln (-n \cdot \bar{k})$, and the one on the bottom contributes $2 a_{2}$ with $a_{2}=\ln (s \cdot \bar{k})$. We have $\eta=-a_{1}+a_{2}$, and once more verify that the gravitational action is additive. Finally, in panel $e$, we have that the contribution from the spacelike/timelike joint is $-2 \eta$, with $\eta=\ln (-n \cdot k)-\ln (-s \cdot k)$, again from Eq. 2.97). In this case the null joint on the bottom gives $-2 a_{1}$ with $a_{1}=\ln (-n \cdot k)$, while the one on the top gives $2 a_{2}$ with $a_{2}=\ln (-s \cdot k)$. We have $-\eta=-a_{1}+a_{2}$, as required by additivity.

The spacelike and timelike rules can also handle the case depicted in panel $a$ of Fig. 11. Here the composite figure describes a null/timelike joint giving rise to a joint term $-2 a_{\text {comp. }}$. The null/spacelike joint on the top provides a contribution $-2 a_{\text {top }}$, while the spacelike/timelike joint at the bottom contributes $2 \eta$ with $\eta=\ln (-n \cdot k)-\ln (-s \cdot k)$ from Eq. 2.97). Here the timelike rule makes the assignment $a_{\text {comp }}=\ln (-s \cdot k)$, while the spacelike rule gives $a_{\text {top }}=\ln (-n \cdot k)$. We have that $-a_{\text {comp }}=-a_{\text {top }}+\eta$, and once more we find that the rules ensure the proper additivity of the gravitational action.

The spacelike and timelike rules, however, are not sufficient to handle the case illustrated in panel $b$ of Fig. 11: for this and similar cases we require the null rule. The composite figure represents a null/null joint with joint term $-2 a_{\text {comp. }}$. The null/spacelike joint at the top comes with $-2 a_{\text {top }}$, while the joint at the bottom comes with $-2 a_{\text {bot }}$. The spacelike rule makes the assignments $a_{\text {top }}=\ln (-n \cdot k)$ and $a_{\text {bot }}=\ln (-n \cdot \bar{k})$. We may decompose $n^{\alpha}$ in the null basis provided by $k^{\alpha}$ and $\bar{k}^{\alpha}$ and conclude that $(-n \cdot k)(-n \cdot \bar{k})=-\frac{1}{2} k \cdot \bar{k}$. According to this, we have that $a_{\mathrm{top}}+a_{\mathrm{bot}}=a_{\mathrm{comp}}$, as required by additivity.

Many other examples could be constructed. In all such cases the three additivity rules formulated in this section are sufficient to restore the additivity of the gravitational action when null boundaries are involved. We not that 

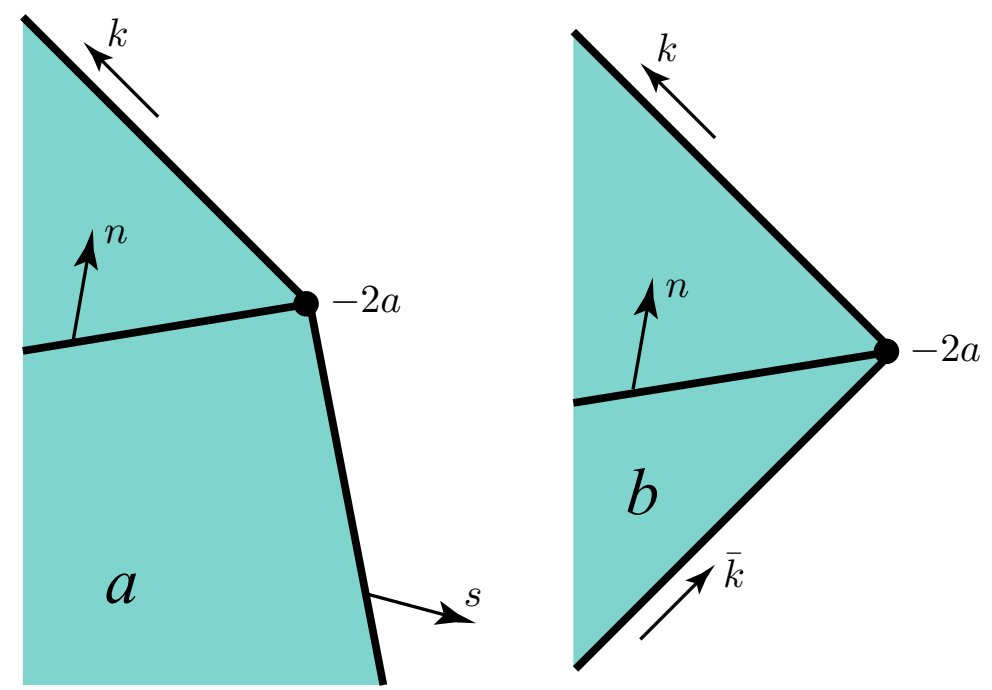

FIG. 11. Composition of boundary actions. In panel $a$, a null/timelike joint is obtained by taking the union of a null/spacelike joint with a spacelike/timelike joint. In panel $b$, a null/null joint is the union of two null/spacelike joints.

these additivity rules do nothing, however, to restore additivity in the problematic case of timelike joints identified by Brill and Hayward [25].

\section{RATE OF CHANGE OF THE GRAVITATIONAL ACTION FOR ADS BLACK HOLES}

In this section we return to the "complexity equals action" conjecture introduced in [7, 8. As described previously, this conjecture leads one to consider the action of regions of asymptotically AdS spacetimes with null boundaries. Our considerations of boundary terms in Sec. II allow us to provide a precise definition for the gravitational action $S=S_{\mathscr{V}}+S_{\partial \mathscr{V}}$ when the region $\mathscr{V}$ possesses a boundary with one or several null segments, which was lacking in [7, 8]. Hence we are able to provide a careful examination of the results presented there.

As we have seen, $S$ contains contributions from $\mathscr{V}$, from the piecewise smooth portions of the boundary $\partial \mathscr{V}$, and from the joints $\mathcal{B}$ where these portions are joined together. However, in general the resulting gravitational action $S$ is ambiguous for a given spacetime, because the contribution from each null segment of the boundary depends on an arbitrary choice of parameterization, and because the contribution from each null joint is the integral of an arbitrary scalar field $a$. The first source of ambiguity, the one associated with the choice of parametrization, is naturally tamed by declaring that all null segments shall be affinely parametrized. This choice ensures that $\kappa=0$ and that the null segments make no contribution to the gravitational action. Then the additivity rules formulated in Sec. [IH] allow us to eliminate (much of) the arbitrariness associated with the null joints. We adopt both of these conventions in the following calculations, but we must acknowledge that these choices do not completely eliminate the ambiguities. In particular, there remains the freedom to rescale the affine parameter $\lambda$ by a constant factor on each generator of the null boundaries, which in turn will rescale the contribution of the corresponding joint terms. We fix this remaining ambiguity by imposing a fixed normalization condition of the null normals at the asymptotic AdS boundary. While this normalization is again an arbitrary choice, such a condition must be imposed if one is going to compare the actions of different regions (potentially in different spacetimes) in a meaningful way.

We wish to exploit our precise definition of the gravitational action to calculate how $S$ changes with time when evaluated for a Wheeler-deWitt patch of a black hole in anti-de Sitter spacetime. This computation was first presented by Brown et al [8] using an incomplete specification of the action, and the analysis there might be viewed as questionable. However, we shall show that our more complete and rigorous methods produce precisely the same answer: In particular, for a Schwarzschild-anti de Sitter black hole at late times $t$,

$$
\frac{d S}{d t}=32 \pi G_{\mathrm{N}} M
$$

where $M$ is the total mass-energy assigned to the black hole. We recall that our convention for the gravitational action 
$S$ omits the usual factor of $1 /\left(16 \pi G_{\mathrm{N}}\right)$, i.e., $S=16 \pi G_{\mathrm{N}} I$. Hence, in the more usual convention, this equation would read $d I / d t=2 M$, and with Eq. (3.1) we have therefore reproduced the elegant universal result of [7, 8]. In fact, our calculations extend the previous analysis to include black holes with planar and hyperbolic horizons. We conclude this section by reconsidering the case of charged black holes in anti-de Sitter spacetime and again, our analysis reproduces the results of [7, 8].

\section{A. Schwarzschild-anti de Sitter spacetime}

We express the metric of an $(n+2)$-dimensional SAdS spacetime as follows:

$$
d s^{2}=-f(r) d t^{2}+\frac{d r^{2}}{f(r)}+r^{2} d \Sigma_{k, n}^{2}, \quad \text { with } \quad f(r)=\frac{r^{2}}{L^{2}}+k-\frac{\omega^{n-1}}{r^{n-1}} .
$$

Here, $L$ is the AdS curvature scale and $k=\{+1,0,-1\}$ denotes the curvature of the $n$-dimensional line-element $d \Sigma_{k, n}^{2}$, given by

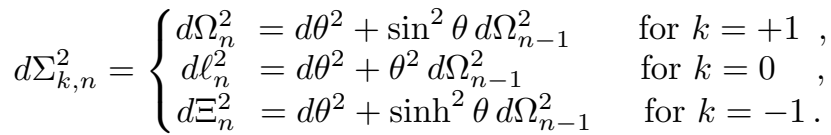

Here, $d \Omega_{n}^{2}$ is the standard metric on a unit $n$-sphere, while $d \ell_{n}^{2}$ is the flat metric on $R^{n}$ (with dimensionless coordinates) and $d \Xi_{n}^{2}$ is the metric on an $n$-dimensional hyperbolic 'plane' with unit curvature. In all three cases, the metric of Eq. (3.2) is a solution of Einstein's equations with a negative cosmological constant, i.e.,

$$
R_{\mu \nu}-\frac{1}{2} R g_{\mu \nu}+\Lambda g_{\mu \nu}=0 \quad \text { with } \quad \Lambda=-\frac{n(n+1)}{2 L^{2}} .
$$

Each of these solutions can be represented by the same Penrose diagram, as shown in Fig. 12. In particular, the black holes corresponding to $k=\{+1,0,-1\}$ have spherical, planar, and hyperbolic horizons, respectively. Of course, these geometries are also static with Killing vector $\partial_{t}$.

The parameter $\omega$ is related to the position of the event-horizon $r_{\mathrm{H}}$ by 13

$$
\omega^{n-1}=r_{\mathrm{H}}^{n-1}\left[\left(r_{\mathrm{H}} / L\right)^{2}+k\right] .
$$

The total mass-energy of the spacetime is given by [30, 32 ]

$$
M=\frac{n \Omega_{n, k}}{16 \pi G_{\mathrm{N}}} \omega^{n-1},
$$

where $\Omega_{n, k}$ denotes the (dimensionless) volume of the corresponding spatial geometry. Hence, for $k=+1$ we have the volume of a unit $n$-sphere, $\Omega_{n,+1}=2 \pi^{(n+1) / 2} / \Gamma\left(\frac{n+1}{2}\right)$, while for $k=0$ and -1 , we implicitly introduce an infrared regulator to produce a finite volume.

For our calculations it is useful to introduce the null coordinates $u$ and $v$, defined by

$$
d u:=d t+f^{-1} d r, \quad d v:=d t-f^{-1} d r .
$$

Integrating these relations yields the "infalling" null coordinate $u=t+r^{*}(r)$ and the "outgoing" null coordinate $v=t-r^{*}(r)$, where $r^{*}(r):=\int f^{-1} d r$. The metric becomes

$$
d s^{2}=-f d u^{2}+2 d u d r+r^{2} d \Sigma_{k, n}^{2}
$$

or

$$
d s^{2}=-f d v^{2}-2 d v d r+r^{2} d \Sigma_{k, n}^{2}
$$

when expressed in terms of the null coordinates. For the three choices $(t, r),(u, r)$, and $(v, r)$ we have that

$$
\int \sqrt{-g} d^{n+2} x=\Omega_{n, k} \int r^{n} d r d w,
$$

where $w=\{t, u, v\}$.

${ }^{13}$ Let us note that with $k=-1$, this mass parameter vanishes when $r_{\mathrm{H}}=L$, but a smooth horizon remains for smaller values of $r_{\mathrm{H}}$ in the range $\frac{n-1}{n+1} \leq \frac{r_{\mathrm{H}}^{2}}{L^{2}}<1$, in which case the mass parameter becomes negative [30, 31]. However, in this regime, the causal structure of the black hole takes the form shown in Fig. 13 with an outer and an inner horizon. Hence the calculation of $d I / d t$ in section IIIB is restricted to $r_{\mathrm{H}}>L$ when $k=-1$. We thank Shira Chapman and Hugo Marrochio for this observation. 

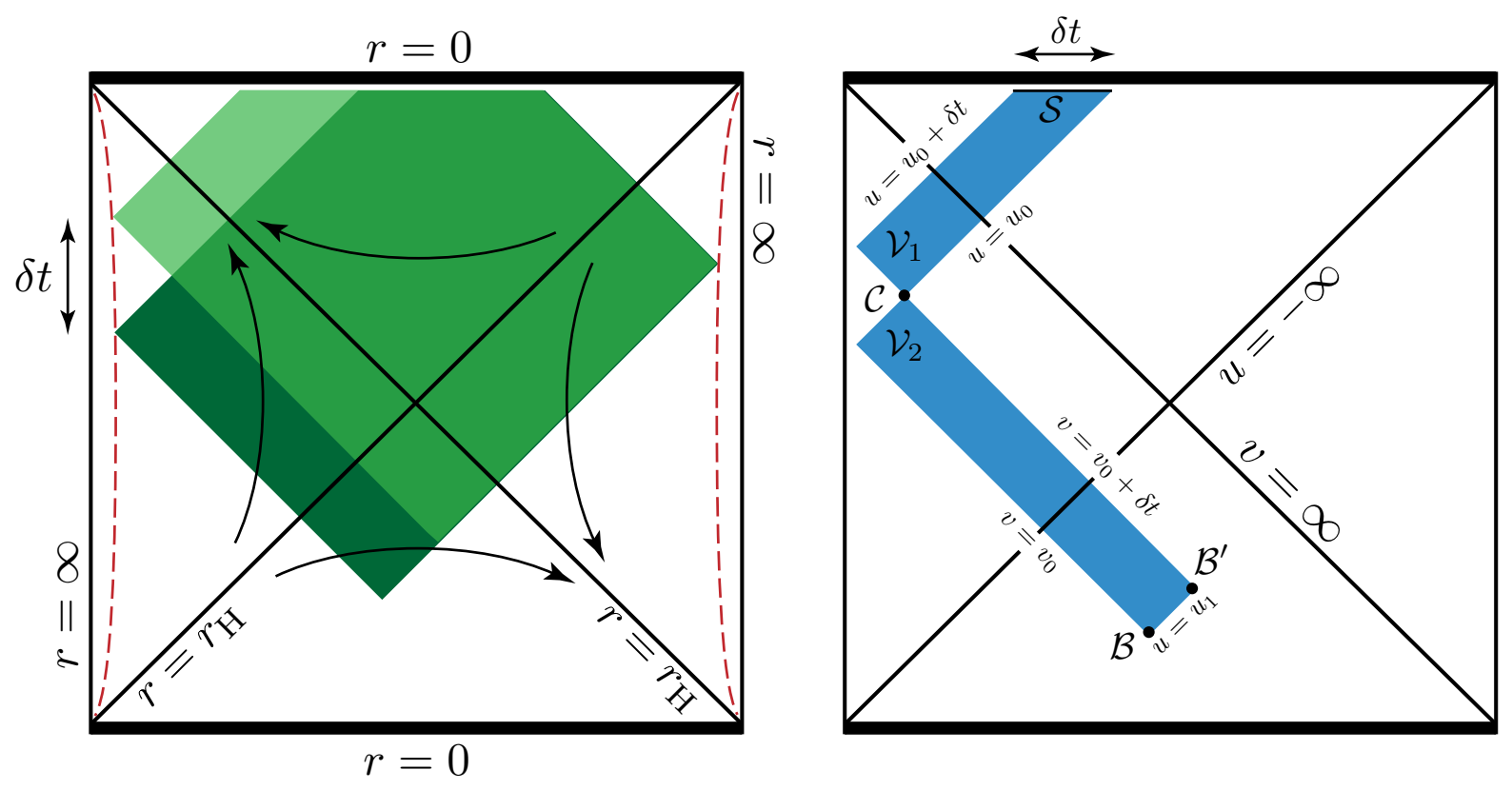

FIG. 12. Wheeler-deWitt patch of a Schwarzschild-anti de Sitter spacetime. On the left panel, the patch at coordinate time $t_{0}$ is shown in dark color, and the patch at time $t_{0}+\delta t$ is shown in light color. The difference between the two patches is shown on the right panel. In the left panel, the curved arrows indicate the flow of the Killing vector $\partial_{t}$ in each of the quadrants of the Penrose diagram. Further the red dashed curves indicate the cut-off surfaces at $r=r_{\max }$ near the asymptotic AdS boundaries.

\section{B. Wheeler-deWitt patch}

We consider the Wheeler-deWitt (WdW) patches of a Schwarzschild-anti-de Sitter spacetime illustrated in Fig. 12. As described in Sec. I. the corresponding action $S\left(t_{\mathrm{L}}, t_{\mathrm{R}}\right)$ depends on the choice of the time slice on the left and right boundaries [7, 8. As shown in the figure, the Killing vector corresponding to time translations in Eq. (3.2) generates an upward (downward) flow in the asymptotic region on the left (right), and hence the action is invariant upon shifting the time slices as $S\left(t_{\mathrm{L}}+\delta t, t_{\mathrm{R}}-\delta t\right)=S\left(t_{\mathrm{L}}, t_{\mathrm{R}}\right)$. Instead we will fix the time on the right boundary and only vary the asymptotic time slice on the left-hand side. In particular, we will compare the actions for the two WdW patches shown on the left panel of the figure. For the first, shown in dark color, the time on the left boundary is $t_{0}$ and we denote $S\left(t_{0}\right)$ the action evaluated for this patch. The asymptotic time for the second WdW patch, shown in light color, is translated slightly with respect to the first by $\delta t$, and the action evaluated for this patch is denoted $S\left(t_{0}+\delta t\right)$. These two actions contain contributions from the interior of the corresponding patches, the bounding surfaces, and the joints between them. Our aim will be to evaluate the difference $\delta S:=S\left(t_{0}+\delta t\right)-S\left(t_{0}\right)$.

We are considering "late times," and so both patches reach the spacelike singularity that defines the future boundary of the Penrose diagram - but they do not touch the past singularity. That is, the patches are bounded by a spacelike surface near the future singularity at $r=0$, and by four null segments extending (almost) all the way to the AdS boundaries at $r=\infty$; in fact, these must be truncated to regulate the gravitational action evaluated for these WdW patches. Note that this is a standard issue in holographic calculations [9] and the standard procedure is to evaluate the quantity of interest with a (timelike) cut-off surface at some large radius. In particular, one might choose the latter to be $r=r_{\max }=L^{2} / \delta$, in which case $\delta$ plays the role of a short distance cut-off in the boundary theory. Typically, holographic calculations are employed to evaluate UV-safe quantities, such as correlation functions, and so one is able to take the limit $\delta \rightarrow 0$ at the end of the calculation [9. In particular, in a standard calculation of the renormalized action, one can introduce a finite set of boundary counterterms which then yield a finite result in this limit 30]. However, such an approach does not yield a finite result for the action of a WdW patch [27, 33. One might therefore interpret the divergence in the action here as being related to the complexity required to establish correlations at arbitrarily short distance scales in the state of the boundary theory. In this way, the divergences found here would be similar to those found in holographic calculations of entanglement entropy [34, 35. For simplicity, our approach to regulating the action will be to let the null sheets defining the boundaries of the WdW patch originate slightly inside the AdS boundary, i.e., , at $(t, r)=\left(t_{0}, r_{\max }\right)$. An alternative approach would be to let the null sheets originate at $(t, r)=\left(t_{0}, \infty\right)$ but truncate the region on which we are evaluating the action at the cut-off surface, 
$r=r_{\max }$. These different choices for the regulator do not significantly change the result for the gravitational action, and both approaches yield the same results in the following; we will return to these issues in [27, 33.

As noted above, another important ingredient in our computations is that the vectors defining the null boundaries of the WdW patch will be normalized in precisely the same way at the asymptotic AdS boundary. While in different contexts we may make different choices for this normalization, here we must fix the normalization in order to compare the action of different WdW patches in a meaningful way. For example, in the following we choose $k \cdot \hat{t}_{L}=-c$, where $k$ is the (future-directed) normal to the past null boundary of the left-hand side of Fig. $12 \hat{t}_{L}=\partial_{t}$ is the asymptotic Killing vector which is normalized to describe the time flow in the left boundary theory, and $c$ is an arbitrary (positive) constant. If instead we allowed the latter to be a function of time, i.e., $k \cdot \hat{t}_{L}=c(t)$, we could produce whatever answer we might desire for the difference $\delta S$ and hence for the time derivative in Eq. (3.1). It is only with a fixed constant $c$ that a meaningful result is produced; we return to this issue in section IV

Now to compute $\delta S=S\left(t_{0}+\delta t\right)-S\left(t_{0}\right)$, we recall that with an affine parametrization for each null surface, these make no contribution to the action and thus to $\delta S$. Further, we observe that the left null joint at $r=r_{\max }$ for $S\left(t_{0}+\delta t\right)$, is simply related to the one for $S\left(t_{0}\right)$ by a time translation; given our fixed normalization of the corresponding null normals, the corresponding joint contributions are identical, and they therefore make no contribution to $\delta S$. A similar conclusion holds for the joints linking the incoming null segment to the spacelike surface near the singularity. Consequently the computation of $\delta S$ relies only on the pieces illustrated on the right panel of Fig. 12 We have the volume contributions from the regions $\mathscr{V}_{1}$ and $\mathscr{V}_{2}$, the surface contribution from the spacelike segment $\mathcal{S}$, and the joint contributions from the $n$-surfaces $\mathcal{B}$ and $\mathcal{B}^{\prime}$. All told, we have that

$$
\delta S=S_{\mathscr{V}_{1}}-S_{\mathscr{V}_{2}}-2 \int_{\mathcal{S}} K d \Sigma+2 \oint_{\mathcal{B}^{\prime}} a d S-2 \oint_{\mathcal{B}} a d S,
$$

where $d \Sigma$ is a volume element on $\mathcal{S}$, and $d S$ is a surface element on $\mathcal{B}$ and $\mathcal{B}^{\prime}$.

\section{Calculation of $\delta S$}

We first evaluate the volume contribution

$$
S_{\mathscr{V}}=\int_{\mathscr{V}}(R-2 \Lambda) \sqrt{-g} d^{n+2} x
$$

for the regions $\mathscr{V}_{1}$ and $\mathscr{V}_{2}$ depicted on the right panel of Fig. 12. By virtue of the Einstein field equations, $R=$ $2(n+2) \Lambda / n$, and the integrand is the constant

$$
R-2 \Lambda=-\frac{2(n+1)}{L^{2}} .
$$

We begin with the computation for $\mathscr{V}_{1}$, and next turn to $\mathscr{V}_{2}$.

As shown in Fig. 12, the past and future null boundaries on the left of the first WdW patch are labelled by $u=u_{0}$ and $v=v_{0}$, respectively. These null boundaries become $u=u_{0}+\delta t$ and $v=v_{0}+\delta t$ for the second, shifted WdW patch. Hence the region $\mathscr{V}_{1}$ is bounded by the null surfaces $u=u_{0}, u=u_{0}+\delta t, v=v_{0}+\delta t$, as well as the spacelike surface $r=\epsilon \ll r_{\mathrm{H}}$. The volume integral is best performed in the $(u, r)$ coordinate system, which is regular throughout the region; in this system the surface $v=v_{0}+\delta t$ is described by $r=\rho(u)$, with $\rho(u)$ defined implicitly by $r^{*}(\rho)=\frac{1}{2}\left(v_{0}+\delta t-u\right)$. Making use of Eq. 3.10), we have that

$$
S_{\mathscr{V}_{1}}=-\frac{2(n+1)}{L^{2}} \Omega_{n, k} \int_{u_{0}}^{u_{0}+\delta t} d u \int_{\epsilon}^{\rho(u)} r^{n} d r=-\frac{2 \Omega_{n, k}}{L^{2}} \int_{u_{0}}^{u_{0}+\delta t} d u \rho^{n+1}(u)
$$

where we have neglected the $\epsilon^{n+1}$ term that was to be subtracted from $\rho^{n+1}$ in the final integrand.

As also shown in the figure, the region $\mathscr{V}_{2}$ is bounded by the null surfaces $u=u_{0}, u=u_{1}, v=v_{0}$, and $v=v_{0}+\delta t$. In this case, the volume integral is most easily performed in the $(v, r)$ coordinates, in which the surfaces $u=u_{0,1}$ are described by $r=\rho_{0,1}(v)$, with $r^{*}\left(\rho_{0,1}\right)=\frac{1}{2}\left(v-u_{0,1}\right)$. Then we have

$$
S_{\mathscr{V}_{2}}=-\frac{2(n+1)}{L^{2}} \Omega_{n, k} \int_{v_{0}}^{v_{0}+\delta t} d v \int_{\rho_{1}(v)}^{\rho_{0}(v)} r^{n} d r=-\frac{2 \Omega_{n, k}}{L^{2}} \int_{v_{0}}^{v_{0}+\delta t} d v\left[\rho_{0}^{n+1}(v)-\rho_{1}^{n+1}(v)\right] .
$$

Now we perform the change of variables $u=u_{0}+v_{0}+\delta t-v$ in the integral for $S_{\mathscr{V}_{1}}$, combine it with the integral for $S_{\mathscr{V}_{2}}$, and notice that the terms involving $\rho(u)$ and $\rho_{0}(v)$ cancel out. This cancellation was to be expected, because the 
portion of $\mathscr{V}_{1}$ below the future horizon and the portion of $\mathscr{V}_{2}$ above the past horizon have equal volumes, as noted in [8]. Taking this property into account, the radial integral for $S_{\mathscr{V}_{1}}$ could have been limited to the interval $\epsilon<r<r_{\mathrm{H}}$, and the integral for $S_{\mathscr{V}_{2}}$ could have been limited to $r_{\mathcal{B}}<r<r_{\mathrm{H}}$; the dependence of each term on $r_{\mathrm{H}}$ would have similarly cancelled out in the difference $S_{\mathscr{V}_{1}}-S_{\mathscr{V}_{2}}$. In any event, we are left with

$$
S_{\mathscr{V}_{1}}-S_{\mathscr{V}_{2}}=-\frac{2 \Omega_{n, k}}{L^{2}} \int_{v_{0}}^{v_{0}+\delta t} d v \rho_{1}^{n+1}(v),
$$

with the function $\rho_{1}$ varying from $r_{\mathcal{B}}$ to $r_{\mathcal{B}^{\prime}}$ as $v$ increases from $v_{0}$ to $v_{0}+\delta t$. This is a small variation in the radius, i.e., $r_{\mathcal{B}^{\prime}}=r_{\mathcal{B}}+O(\delta t)$, and hence the volume contribution to $\delta S$ is simply

$$
S_{\mathscr{V} / 1}-S_{\mathscr{V} / 2}=-\frac{2 \Omega_{n, k}}{L^{2}} r_{\mathcal{B}}^{n+1} \delta t
$$

We next evaluate the surface contribution to $\delta S$, given by $-2 \int_{\mathcal{S}} K d \Sigma$, where $\mathcal{S}$ is the boundary segment given by the spacelike hypersurface $r=\epsilon$. The (future-directed) unit normal to any surface $r=$ constant inside the future horizon is given by $n_{\alpha}=|f|^{-1 / 2} \partial_{\alpha} r$. The extrinsic curvature is then

$$
K=\nabla_{\alpha} n^{\alpha}=-\frac{1}{r^{n}} \frac{d}{d r}\left(r^{n}|f|^{1 / 2}\right),
$$

and the volume element becomes

$$
d \Sigma=\Omega_{n, k}|f|^{1 / 2} r^{n} d t
$$

after integrating over the "angular" variables described by Eq. 3.3. Letting $r=\epsilon \ll r_{\mathrm{H}}$ and then approximating $f \simeq-(\omega / r)^{n-1}$, we find that

$$
-2 \int_{\mathcal{S}} K d \Sigma=(n+1) \Omega_{n, k} \omega^{n-1} \delta t .
$$

Given the proximity of $\mathcal{S}$ to the spacelike singularity at $r=0$, it is remarkable that the answer turns out to be finite and independent of $\epsilon$. This occurs because the divergence in $K$ is precisely compensated for by the vanishing of $d \Sigma$. We return to discuss this point in section IV.

We next turn to the joint terms $\pm 2 \oint$ adS contributed by the $n$-surfaces $\mathcal{B}$ and $\mathcal{B}^{\prime}$. The null rule formulated in Sec. 【H states that

$$
a=\ln \left(-\frac{1}{2} k \cdot \bar{k}\right)
$$

where $k^{\alpha}$ is the (future-directed) null normal to the left-moving null hypersurfaces, i.e., on which $v=v_{0}$ and $v_{0}+\delta t$, while $\bar{k}^{\alpha}$ is the (future-directed) null normal to the right-moving surface, on which $u=u_{1}$.

Our convention was to choose the vectors $k^{\alpha}$ and $\bar{k}^{\alpha}$ to be affinely parametrized, and suitable expressions are

$$
k_{\alpha}=-c \partial_{\alpha} v=-c \partial_{\alpha}\left(t-r^{*}\right), \quad \bar{k}_{\alpha}=\bar{c} \partial_{\alpha} u=\bar{c} \partial_{\alpha}\left(t+r^{*}\right)
$$

where $c$ and $\bar{c}$ are arbitrary (positive) constants. This choice implements the asymptotic normalizations $k \cdot \hat{t}_{L}=-c$ and $\bar{k} \cdot \hat{t}_{R}=-\bar{c}$, where $\hat{t}_{L, R}$ are the asymptotic Killing vectors which are normalized to describe the time flow in the left and right boundary theories, respectively. With these choices, we have that $k \cdot \bar{k}=2 c \bar{c} / f$, so that

$$
a=-\ln \left(\frac{-f}{c \bar{c}}\right) .
$$

With the above expression, we find that

$$
2 \oint_{\mathcal{B}^{\prime}} a d S-2 \oint_{\mathcal{B}} a d S=2 \Omega_{n, k}\left[h\left(r_{\mathcal{B}^{\prime}}\right)-h\left(r_{\mathcal{B}}\right)\right]
$$

where $h(r):=-r^{n} \ln (-f / c \bar{c})$.

To express this result in its final form, we perform a Taylor expansion of $h(r)$ about $r=r_{\mathcal{B}}$. Because the displacement is in a direction of increasing $v$, we have that $d u=0, d v=\delta t$, and $d r=-\frac{1}{2} f \delta t$. This gives us

$$
h\left(r_{\mathcal{B}^{\prime}}\right)-h\left(r_{\mathcal{B}}\right)=-\left.\frac{1}{2} f \frac{d h}{d r}\right|_{r=r_{\mathcal{B}}} \delta t=\left.\frac{1}{2}\left[r^{n} \frac{d f}{d r}+n r^{n-1} f \ln \left(\frac{-f}{c \bar{c}}\right)\right]\right|_{r=r_{\mathcal{B}}} \delta t
$$


and then

$$
2 \oint_{\mathcal{B}^{\prime}} a d S-2 \oint_{\mathcal{B}} a d S=\left.\Omega_{n, k}\left[r^{n} \frac{d f}{d r}+n r^{n-1} f \ln \left(\frac{-f}{c \bar{c}}\right)\right]\right|_{r=r_{\mathcal{B}}} \delta t .
$$

Combining Eqs. 3.17), 3.20), and (3.26), we arrive at

$$
\delta S=\left.\Omega_{n, k}\left[-\frac{2 r^{n+1}}{L^{2}}+(n+1) \omega^{n-1}+r^{n} \frac{d f}{d r}+n r^{n-1} f \ln \left(\frac{-f}{c \bar{c}}\right)\right]\right|_{r=r_{\mathcal{B}}} \delta t
$$

for the change in gravitational action when the left time slice of the WdW patch is translated by $\delta t$. Making use of the explicit expression for $f$, this expression implies that

$$
\frac{d S}{d t}=\left.2 n \Omega_{n, k} \omega^{n-1}\left[1+\frac{1}{2}\left(\frac{r}{\omega}\right)^{n-1} f \ln \left(\frac{-f}{c \bar{c}}\right)\right]\right|_{r=r_{\mathcal{B}}} .
$$

When this is evaluated at late times, $r_{\mathcal{B}}$ approaches $r_{\mathrm{H}}, f$ approaches zero, and we see that $d S / d t$ rapidly approaches the asymptotic constant $2 n \Omega_{n} \omega^{n-1}$. Recalling Eq. 3.6 for the mass-energy of the SAdS spacetime, this is

$$
\frac{d S}{d t}=32 \pi G_{\mathrm{N}} M
$$

at late times. In the more usual convention in which the gravitational action is $I:=S /\left(16 \pi G_{\mathrm{N}}\right)$, this is $d I / d t=2 M$, precisely the same result reported in Brown et al [7, 8. We might add that the calculations there focused on the case of spherical black holes, i.e., $k=+1$. Our analysis shows that the same simple result applies also for planar and hyperbolic horizons, i.e., $k=0$ and -1 .

\section{Comparison with Brown et al}

It is remarkable that the two very different methods of calculating $d I / d t$ should produce precisely the same outcome, given how the accounting of various contributions to the gravitational action differs in each method. It is interesting to examine in detail how each contribution to the action appears in the calculation of $d I / d t$ in [7, 8] and compare with our results:

First, Brown et al implicitly assume that the gravitational action is additive. They use this property to divide the $\mathrm{WdW}$ patches at $t_{0}$ and $t_{0}+\delta t$ into various subregions and evaluate $\delta S$ in terms of the action evaluated for each of the subregions. In the end, they essentially focus on two regions, $\mathcal{V}_{1}$ and $\mathcal{V}_{2}$ on the right panel of Fig. 12 but each of these is further divided into the portion outside of the horizon and that behind the horizon. We note that only spacelike joints arise in subdividing the WdW patches there; as we discussed, with appropriate choices for the boundary terms, the gravitational action will indeed be additive. Further, we observe that (segments of) the future and past horizons now play the role of boundary surfaces for these various subregions. These (null) surfaces did not appear in our calculations because we did not subdivide the $\mathrm{WdW}$ patches. One may worry that the final results will depend on choices made, e.g., in defining the parametrization of these null surfaces. To answer this we make two observations: First, in general, any internal boundary surface will be common to two neighbouring subregions, so as long as the common boundary is described consistently in evaluating the action of these two subregions (e.g., they are assigned the same null normal $k^{\alpha}$ ), the corresponding boundary contributions will cancel when the actions are added to evaluate the full action of the complete WdW patch. Thus, the choices made in describing such internal boundary surfaces will never affect the final result. Second, for the particular case considered here, the internal boundary surfaces are segments of a Killing horizon, i.e., they are stationary null boundary surfaces. As discussed below Eq. 2.70 and in Appendix A, such stationary null boundaries are distinguished because the corresponding contributions to the gravitational action are not ambiguous. This point will play an important role in the following.

In the calculation presented in [7, 8], the authors argue that the time translation symmetry of the geometry ensures that the contributions to $S\left(t_{0}+\delta t\right)$ and $S\left(t_{0}\right)$ from the portions of the corresponding WdW patches outside of the horizon will cancel in the difference $\delta S$. While we agree with this conclusion, assuming the reasonable choices described above for the boundary terms, we would like to point out a subtlety having to do with the boundary contributions coming from the horizon. In particular, it is not true that the boundary contribution coming from the future horizon (or from the past horizon) is identical for these two exterior regions. Instead, the two actions cancel because the contribution from the segment between $u_{0}$ and $u_{0}+\delta t$ on the future horizon cancels that from the segment between $v_{0}$ and $v_{0}+\delta t$ on the past horizon. The simplest way to see that these two contributions match is to note that since 
the geometry is static, it is invariant under an inversion of the time coordinate. Hence, inverting $t$ about the time slice $t_{0}+\frac{1}{2} \delta t$ maps these boundary segments on the two horizons into one another. Further, in comparing these two null segments, it is important that there is no ambiguity in their contributions to the gravitation action, since both are part of a stationary horizon, as discussed above.

Next, Brown et al consider the portion of $\mathcal{V}_{2}$ which lies behind the past horizon (see Fig. 12). At late times, the radial coordinate is essentially constant throughout this region and so the geometry reduces to the direct product of a constant transverse space, i.e., the $n$-dimensional geometry described by Eq. (3.3), and the exponentially small two-dimensional geometry extending in the $r$ and $t$ (or $u$ and $v$ ) directions. Since the transverse geometry is constant, the authors argue that by applying the two-dimensional Gauss-Bonnet theorem [36, this region does not contribute to the time dependence of the WdW patch, although they acknowledge that there may be subtleties in this argument related to regulating the gravitational action. Our construction seems to eliminate this issue or at least, relates any question about the UV divergences in the complexity to the behaviour of the geometry and the WdW patch near the asymptotic boundary [33. Let us add that the analysis of [36] implicitly introduces a new imaginary contribution to the null joint terms, which violates the additivity of the gravitational action. However, these imaginary terms do not affect the result for $d I / d t$ - see section IV for further discussion. From our perspective, it is the proximity of the $u=u_{1}$ surface to the past horizon that ensures the cancellation of the corresponding contributions. For example, if the generators of the past horizon were affinely parametrized, the only contributions to the gravitational action of this region $\sqrt{14}$ would come from from the joints on the boundary, i.e., $\mathcal{B}, \mathcal{B}^{\prime}$ and the intersections of $v=v_{0}$ and $v=v_{0}+\delta t$ with the past horizon. Then because of the proximity of the $u=u_{1}$ boundary and the past horizon, evaluating the joint terms at $\mathcal{B}$ and $\mathcal{B}^{\prime}$ yields essentially the same result as those on the horizon, up to an overall sign. We return to this point below.

Lastly, Brown et al consider the portion of $\mathcal{V}_{1}$ which lies behind the future horizon (see Fig. 12) and whose action then gives the entire result for $\delta S$. There are three contributions: $i$ ) the volume integral of the Einstein-Hilbert action; ii) the boundary integral of the Gibbons-Hawking-York (GHY) term on a spacelike surface near the singularity at $r=0$; and $i i i)$ the GHY term evaluated on a spacelike surface just inside the horizon which is then taken to approach $r=r_{\mathrm{H}}$. The first two contributions also appear in our calculations ${ }^{15}$, but the third term may seem suspect in view of our discussion of ambiguities in taking the null limit of spacelike or timelike surfaces. However, here the limit is taken to a stationary null surface, and there is no such ambiguity. A caveat is that there is no ambiguity for the sum of the boundary and joint terms evaluated on a stationary null surface in this way. Brown et al assume that the joint terms cancel between the two ends of the null segment, which is not a priori clear from our perspective. On the other hand, a careful analysis along the lines of those given for the first example in Appendix A shows that this cancellation indeed occurs.

Hence at a pragmatic level, the key difference between the two calculations is as follows: In the Brown et al computation, an essential contribution to $\delta S$ originates from the segment of the future horizon (between $u=u_{0}$ and $u_{0}+\delta t$ ), which plays no role in our calculation as it appears on a surface that is internal to the WdW patch. On the other hand, our computation features contributions from the joints, $\mathcal{B}$ and $\mathcal{B}^{\prime}$, at the bottom of the WdW patches, which play an inconsequential role in [7, 8. However, when all contributions are combined together, the final result is the same $d I / d t=2 M$ for late times in both cases. It thus follows that these two distinct contributions appearing in the different calculations must in fact be the same. Tracing through the above discussion, we can see the mechanism for this equality. First, because the future horizon is an internal boundary, the boundary contribution of the null segment on the future horizon is the same for the portions of $\mathcal{V}_{1}$ inside and outside of the horizon, up to an overall sign. Then because of the time inversion symmetry (as well as the time translation symmetry) of the geometry, the contribution on the future horizon for the exterior part of $\mathcal{V}_{1}$ can be related to that on the past horizon for the exterior of $\mathcal{V}_{2}$. Again the past horizon is an internal boundary and so the contribution is the same for the corresponding null segment of the portion of $\mathcal{V}_{2}$ inside the horizon. Lastly, we found that the joint contributions from $\mathcal{B}$ and $\mathcal{B}^{\prime}$ match the contribution from the horizon for this portion of $\mathcal{V}_{2}$ inside the horizon. Hence through a series of equalities, we see that the boundary contribution from the segment of the future horizon in the Brown et al computation must be equal to the joint contributions from the bottom of the WdW patch in our computation. Therefore the key difference between the two approaches is largely a matter of accounting, i.e., while our computation directly compared the full $\mathrm{WdW}$ patches at $t=t_{0}$ and $t_{0}+\delta t$, Brown et al begin by subdividing the WdW patches and evaluate the action on a series of subregions. However, as explained above, Brown et al also make a number of assumptions, beginning with additivity of the gravitational action, which we have verified with the detailed considerations in our paper.

\footnotetext{
14 The integral of the Einstein-Hilbert term is negligible because the proper volume of this region is exponentially small.

15 Given our presentation of the calculation of the Einstein-Hilbert term above, this statement may not be immediately clear. However, we observe that at the end of the calculation we set $r_{\mathcal{B}} \simeq r_{\mathrm{H}}$, and so there is essentially no contribution to the volume integral coming from behind the past horizon in $\mathcal{V}_{2}$ - as noted in the previous footnote.
} 


\section{E. Extension to charged black holes}

We next turn our attention to the case of a charged AdS black holes in $n+2$ dimensions ${ }^{16}$ The line element takes the same form as in Eq. (3.2), but with (see, for example, [37]),

$$
f(r)=\frac{r^{2}}{L^{2}}+k-\frac{\omega^{n-1}}{r^{n-1}}+\frac{q^{2}}{r^{2(n-1)}} .
$$

The full solution also includes the Maxwell vector potential, which may be written as

$$
A_{\alpha} d x^{\alpha}=\sqrt{\frac{n}{2(n-1)}}\left(\frac{q}{r_{\mathrm{H}}^{n-1}}-\frac{q}{r^{n-1}}\right) d t
$$

where the constant term is chosen to ensure that $A_{t}$ vanishes at the horizon ${ }^{17}$ The combined metric and vector potential then provide a solution for the Einstein-Maxwell equations resulting from the (bulk) action,

$$
S_{\mathscr{V}}=\int_{\mathscr{V}}\left(R-2 \Lambda-F_{\alpha \beta} F^{\alpha \beta}\right) \sqrt{-g} d^{n+2} x,
$$

where $F_{\alpha \beta}=\partial_{\alpha} A_{\beta}-\partial_{\beta} A_{\alpha}$ is the electromagnetic field strength. Recall that $\Lambda=-n(n+1) /\left(2 L^{2}\right)$. The ADM mass of this charged solution is given by the same expression as in Eq. (3.6), while the charge of the gauge potential is related to the parameter $q$ by

$$
Q=q \sqrt{2 n(n-1)} \frac{\Omega_{n, k}}{8 \pi G}
$$

As before, we wish to calculate the change $\delta S=S\left(t_{0}+\delta t\right)-S\left(t_{0}\right)$ in the total (gravitational plus electromagnetic) action between the two WdW patches displayed in Fig. 13 , where the time slice on the left boundary is shifted slightly by $\delta t$ (and we are considering late times $t_{0}$ ). The details of the calculation are virtually identical to those presented in the preceding subsection, and we can rely on a few key observations to simplify our task. First, the asymptotic joints near the left AdS boundary are related by a time translation, and their contributions cancel out in $\delta S$. Second, by virtue of the additivity of the action we can conclude that the boundaries internal to the regions of interest, e.g., the event horizon, do not contribute to $\delta S$; only the external boundaries are relevant. Third, the external boundaries are all segments of null hypersurfaces, which give no contribution to $\delta S$ (or the individual actions) by virtue of our assumption that the generators are affinely parametrized. As a result, inspection of the right panel of Fig. 13 indicates that in addition to the volume contributions, only the joints $\mathcal{B}, \mathcal{B}^{\prime}, \mathcal{C}$, and $\mathcal{C}^{\prime}$ contribute to $\delta S$.

We begin with the evaluation of the volume contribution to the action from Eq. (3.32) and use

$$
R=\frac{2(n+2)}{n} \Lambda+\frac{n-2}{n} F_{\alpha \beta} F^{\alpha \beta}
$$

which is a consequence of the Einstein equations. As in the uncharged case, only the region inside the future horizon contributes to $\delta S$, because the regions outside the horizon produce canceling contributions, and the region inside the past horizon contributes a negligible term at late times. The remaining contribution is then given by an equation similar to Eq. 3.14, with integration limits given by $r_{+}$and $r_{-}$at late times. We arrive at

$$
\delta S_{\mathscr{V}}=-2 \Omega_{n, k}\left[\frac{r_{+}^{n+1}-r_{-}^{n+1}}{L^{2}}-\frac{q^{2}}{r_{-}^{n-1}}+\frac{q^{2}}{r_{+}^{n-1}}\right] \delta t .
$$

We also rely on the results presented in the preceding section to evaluate the joint contributions to $\delta S$. In this case we find that

$$
\delta S_{\mathcal{B}, \mathcal{B}^{\prime}}=\left.\Omega_{n, k} r^{n} \frac{d f}{d r}\right|_{r_{+}} \delta t, \quad \delta S_{\mathcal{C}, \mathcal{C}^{\prime}}=-\left.\Omega_{n, k} r^{n} \frac{d f}{d r}\right|_{r_{-}} \delta t
$$

\footnotetext{
16 The results presented in this section were independently confirmed by Shira Chapman and Hugo Marrochio (private communication). 17 The latter is required for $A=A_{\alpha} d x^{\alpha}$ to be a well-defined one-form at the bifurcation surface(s).
} 


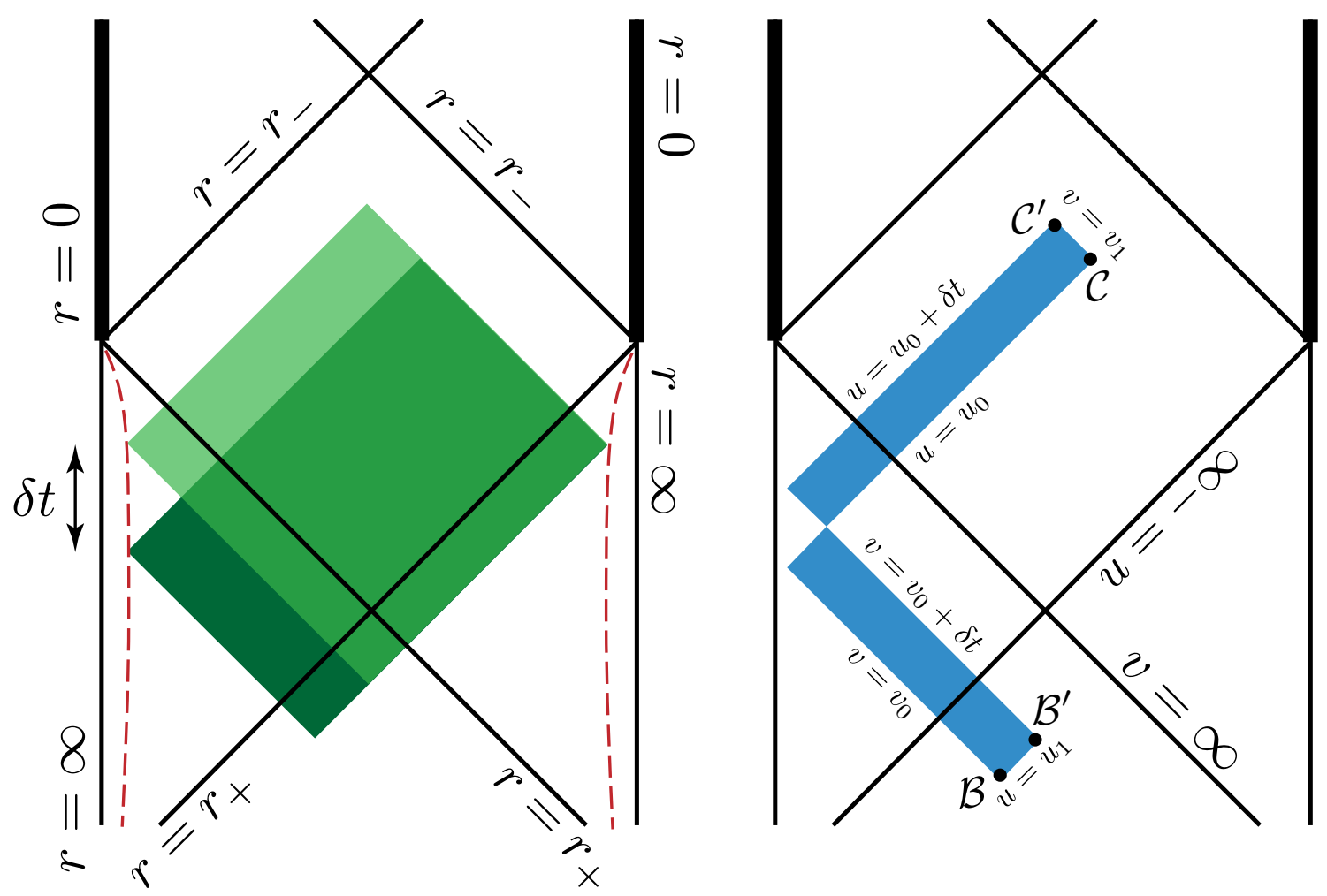

FIG. 13. Wheeler-deWitt patch of a Reissner-Nordström-anti de Sitter spacetime. On the left panel, the patch at coordinate time $t_{L}=t_{0}$ is shown in dark color, and the patch at time $t_{L}=t_{0}+\delta t$ is shown in light color. The difference between the two patches is shown on the right panel. The red dashed curves on the left panel indicate the cut-off surfaces at $r=r_{\text {max }}$ near the asymptotic AdS boundaries.

the relative sign between the two expressions is a consequence of the different signs involved in null-joint terms in the action (as summarized in Fig. 9). The combined joint contributions therefore give

$$
\begin{aligned}
\delta S_{\mathcal{B}, \mathcal{B}^{\prime}}+\delta S_{\mathcal{C}, \mathcal{C}^{\prime}} & =\left.2 \Omega_{n, k}\left(\frac{\omega^{n-1}}{2}-\frac{q^{2}(n-1)}{r^{n-1}}+\frac{r^{n+1}}{L^{2}}\right)\right|_{r_{-}} ^{r_{+}} . \\
& =2 \Omega_{n, k}\left[\frac{q^{2}(n-1)}{r_{-}^{n-1}}-\frac{q^{2}(n-1)}{r_{+}^{n-1}}+\frac{r_{+}^{n+1}-r_{-}^{n+1}}{L^{2}}\right] \delta t .
\end{aligned}
$$

Combining Eqs. (3.35) and (3.37), we arrive at

$$
\delta S=2 n \Omega_{n, k}\left(\frac{q^{2}}{r_{-}^{n-1}}-\frac{q^{2}}{r_{+}^{n-1}}\right) \delta t,
$$

or

$$
\frac{d I}{d t}=n \frac{\Omega_{n, k}}{8 \pi G_{\mathrm{N}}}\left(\frac{q^{2}}{r_{-}^{n-1}}-\frac{q^{2}}{r_{+}^{n-1}}\right) .
$$

This expression agrees with the one reported by Brown et al $[8]$ when $n=2$ and $k=+1$, that is, for a four-dimensional spherical charged AdS black hole. Further, let us observe that when $q \rightarrow 0, r_{-}^{n-1} \rightarrow q^{2} / \omega^{n-1}$ and $r_{+} \rightarrow r_{\mathrm{H}}$, and hence we recover the expected $d I / d t \rightarrow 2 M$ for any number of spacetime dimensions. 


\section{DISCUSSION}

We have presented a complete analysis of the boundary terms required in the action functional of general relativity, paying careful attention to the case of null boundary segments. As we have seen, this case requires the introduction of two new classes of boundary terms, the first on the null segments themselves, and the second on the associated joint terms where the null boundaries intersect other segments. For a typical null segment and its associated joint, we have 18

$$
-2 \int \kappa d S d \lambda+2 \oint a d S
$$

In the first term, $\kappa$ is defined by Eq. 2.34), $k^{\beta} \nabla_{\beta} k^{\alpha}=\kappa k^{\alpha}$, where $k^{\alpha}$ is the (future-directed) null tangent vector along the boundary segment. With $k^{\alpha}=\partial x^{\alpha} / \partial \lambda$ as in Eq. $(2.33), \kappa$ measures the failure of $\lambda$ to be an affine parameter along the null generators of the boundary segment. In section IIF 4, we showed that the function $a$ in the joint terms takes the form

$$
a=\ln |n \cdot k|+a_{0},
$$

where $n^{\alpha}$ is the unit normal to the other boundary segment forming the joint, and $a_{0}$ is an arbitrary scalar whose variation $\delta a_{0}$ is required to vanish.

As discussed in the main text, the null boundary terms (4.1) are ambiguous. In particular, $\kappa$ depends on an arbitrary choice of parametrization for the null generators, i.e., the choice of $\lambda$. Further, there are two distinct ambiguities in the expression (4.2) for $a$. First, the piece $\ln |n \cdot k|$ depends on the arbitrary normalization of the null tangent $k^{\alpha}$, and this ambiguity is again related to the choice of $\lambda \sqrt{19}$ The second term in Eq. 4.2, $a_{0}$, reveals a separate dependence on the choice of the function $\Phi$ defining the boundary surface — see the discussion above Eq. 2.32. Despite these ambiguities, the variation of the boundary terms on the null segments and null joints is well-defined and by construction, it cancels the corresponding total derivative terms coming from the variation of the bulk action. However, evaluating the gravitational action for a particular spacetime geometry will yield different numerical values depending on the different choices in the construction of the boundary terms (4.1).

We might add that there are further ambiguities that are inherent to any variational problem. For example, one can always add a total derivative term to the (bulk) action without affecting the equations of motion, and with a judicious choice, without affecting the vanishing of the boundary variations. However, in general such a term would modify the value of the action when it is evaluated on a particular field configuration. Similarly, one could add boundary terms whose variation vanishes, e.g., because of the boundary conditions imposed on the fields. The scalar $a_{0}$ appearing in the null joint terms 4.2 would be an example of such a boundary term. While we chose this scalar to be a simple constant, the variational principle would remain intact with more complicated choices, e.g., $a_{0} \propto \mathcal{R}$ where $\mathcal{R}$ is the Ricci scalar associated with the induced geometry on the joint. A further example of the latter class would be the term $\int_{\Sigma} \Theta \sqrt{\gamma} d^{2} \theta d \lambda$ introduced on null boundary segments in [6] - see the discussion below Eq. 2.66. In this paper we have proposed what we see as the minimal set of boundary terms for the gravitational action, and we have not considered specious contributions of the above form 20

In the context of the "complexity equals action" conjecture [7, 8], the ambiguities described above may seem to be problematic. However, as emphasized in [7, 8], the circuit complexity of a quantum state is also ambiguous. In particular, it depends on the choice of initial reference state and specific set of quantum gates, with which one acts to construct the desired state. Further, the precise value of the complexity will depend on the tolerance that one introduces to describe the accuracy with which the desired state must be constructed. It would be interesting to draw a more precise connection between the ambiguities described here for the circuit complexity and those described above for the gravitational action.

Now as we discussed, the ambiguities in the gravitational action can be tamed with natural prescriptions. In particular, the reparametrization ambiguity on the null segments can be mitigated by choosing the null generators to be affinely parametrized, and then the corresponding boundary term simply vanishes. Further, in section II H the undetermined functions $a_{0}$ at the null joints were fixed by demanding additivity for the gravitational action. 21 These choices still leave the freedom to rescale the affine parameter along any of the null segments by a constant factor. However, in the context of the AdS/CFT correspondence and evaluating the action on WdW patches, we can remove

18 Here we do not concern ourselves with the precise sign associated with these contributions to the action - see section II or appendix C

19 Note that this ambiguity remains even if a specific prescription is chosen for $\kappa$. That is, if we fix $\kappa$ in Eq. 2.34, we must still specify the initial value for $k^{\alpha}$ in order to solve this differential equation.

20 Appendix B introduces an interesting "specious" boundary term which removes the reparametrization ambiguities on the null segments. We hope to discuss this boundary "counterterm" in greater detail elsewhere 27 .

${ }^{21}$ Implicitly, to produce an additive gravitational action, we are assuming that the same null tangents are used on any common null segments for neighbouring spacetime regions. 
this final ambiguity by imposing a normalization condition on the null normals near the asymptotic AdS boundary; see the discussion towards the end of section IIIB This choice (along with the previous two) allows us to make a meaningful comparison of the action for different WdW patches. In particular, following [7, 8] we evaluated the rate of change of the action for the WdW patch of asymptotically AdS black holes in Sec. III and found the same result reported there, i.e., $d I / d t=2 M$ (for late times and uncharged black holes). Our analysis reveals that this simple result applies to noncompact horizons (i.e., planar and hyperbolic horizons) as well as spherical horizons.

We wish to emphasize that the result $d I / d t=2 M$ for SAdS black holes at late times is very robust, i.e., it does not depend on the specific choices made above to eliminate the ambiguities associated with null boundary terms. Examining each of our choices in reverse order, we see that in Eq. 3.22 , the asymptotic normalization of the null tangents was in fact arbitrary, i.e., $k \cdot \hat{t}_{L}=-c$ and $\bar{k} \cdot \hat{t}_{R}=-\bar{c}$, where $c$ and $\bar{c}$ are arbitrary constants. Our final result for the rate of change of the action was independent of these constants - see Eq. (3.28). We also see that in the joint contributions (4.2), $a_{0}$ could be chosen to be any constant (or in fact, some function of the intrinsic geometry of the null joint) and the precise choice would not modify our result for $d I / d t$. The independence of $a_{0}$ is a consequence of the Killing symmetry $\partial_{t}$ of the black hole spacetime and of the very small difference in the geometries of the joints $\mathcal{B}$ and $\mathcal{B}^{\prime}$ at late times - see Fig. 12. Finally, one can imagine replacing our choice $\kappa=0$ with $\kappa=\kappa_{0}$, where $\kappa_{0}$ is some arbitrary nonvanishing constant on the null boundary segments - in fact, different values of $\kappa_{0}$ might be chosen on the different null boundaries. Our result for $d I / d t$ would again remain unchanged, first because most of the null boundary contributions would simply cancel as a result of the Killing symmetry $\partial_{t}$, and second because at late times, the segment at $u=u_{1}$ is almost a stationary surface, i.e., this null boundary is very close to the past horizon at $u=-\infty-$ again, see Fig. 12 . We must note that this robustness relies on fixing the ambiguities with "reasonable" choices. For example, if one were to choose $c=c(t)$ or $\kappa_{0}=\kappa_{0}(t)$ above, then one would find $d I / d t \neq 2 M$ and the result would depend on the details of the selected functions. However, our perspective is that with such arbitrary (time-dependent) choices, one simply cannot expect to meaningfully compare the action of WdW patches at different times. The significant role of the time-translation Killing symmetry highlighted above suggests the importance of making detailed studies of time-dependent scenarios in the future.

One slightly unsettling feature of the calculation of $d I / d t$ is that an essential contribution comes from the boundary segment near the spacelike singularity at $r=0$, i.e., the boundary segment $\mathcal{S}$ in Fig. 12. This contribution is determined by evaluating the $K$ term on a regulator surface at $r=\epsilon\left(\ll r_{\mathrm{H}}\right)$ and then taking the limit $\epsilon \rightarrow 0$. It is remarkable that the result (3.20) turns out to be finite and independent of $\epsilon$. At a pragmatic level, this occurs because the divergence in $K$ is precisely compensated for by the vanishing of the volume element $d \Sigma$. This precise balance relies on the specific behavior of the metric function $f(r)$ near the singularity, and therefore on the assumed validity of the Einstein equations in this region of spacetime. Of course, this outcome might be regarded with suspicion since UV effects, e.g., higher curvature terms arising as stringy and quantum corrections, are expected to modify the field equations and spacetime geometry in the vicinity of the singularity. However, one might argue that these deviations should be small so long as the regulator scale $\epsilon$ is chosen to be well above the quantum gravity scale, and hence the evaluation of the action should be robust. As a simple test of this reasoning, one might examine how the rate of change 1.1. of the WdW patch action is modified if the regulator is taken to be small but finite. Here one finds

$$
\delta\left(\frac{d I}{d t}\right)=-k \frac{n \Omega_{n, k}}{8 \pi G_{\mathrm{N}}} \epsilon^{n-1}
$$

Hence for a spherical horizon (i.e., $k=+1$ ), the regulator corrections reduce the rate, which seems to align with the conjecture of [7, 8] that there should be a bound $d I / d t \leq 2 M$. However, the rate increases for a hyperbolic horizon (i.e., $k=-1$ ) and the result may seem to contradict those expectations. We should add that preliminary investigations [38, 39] into extending these calculations to (classical) higher curvature theories have cast doubt on the simple argument given above. It is certainly a question which deserves further study.

Future studies of the "complexity equals action" conjecture should examine less symmetric situations, as well. With less symmetry, caustics and crossings will generically appear on the boundary of the WdW patch, i.e., there will be points where the null generators of the boundary of the WdW patch cross each other and the boundary fails to be smooth ${ }^{22}$ Indeed, this situation will arises generically whenever one seeks the action of a spacetime region defined in terms of future- or past-sets.

Our intuition is that locally such crossings will typically have the geometry of a spacelike joint formed by the intersection of two null surfaces, e.g., see [40. We have not proven that this is the only generic case, but it is at least clear that such null-null joints form a broad class of stable crossings. In this case, our results would allow the

22 To clarify our nomenclature, we use "caustic" to refer to the situation where the crossing null rays were only infinitesimally separated in the transverse directions on the boundary, and "crossing" for the case where the null rays were initially widely separated. 
evaluation of the corresponding gravitational action, in that one would simply include an additional joint contribution with $a=\ln \left(-\frac{1}{2} k \cdot \bar{k}\right)$, where $k^{\alpha}$ and $\bar{k}^{\alpha}$ are the null normals on either side of the joint.

A new feature would be that these crossings may terminate at a caustic or at another crossing of lower dimension, for example at a "corner" or joint of codimension-three, i.e., the simultaneous intersection of three segments of the boundary surface. In such cases, one might need to include an additional contribution to the action from the corner or caustic where the null-null joint terminates - see further discussion of such corner contributions below. To evaluate the boundary contribution for a caustic, one approach would be to "regulate" the geometry as follows: First, introduce an additional timelike boundary surface which cuts the endpoints of the crossing out of the boundary and then remove this regulator surface so that the endpoints reappear in a limit. It seems that the regulated geometry in such an approach would also typically involve a codimension-three joint. This provides some motivation to study the boundary terms (if any are needed) for such higher codimension corners, as discussed in the next paragraph.

Our discussion in this paper has focused on the possibility of spacelike joints, or intersections between pairs of boundary surfaces. The case of timelike joints where two timelike boundary surfaces meet was also considered in [4, 25]. Of course, our analysis could be further generalized to consider more complicated intersections involving more than two boundary surfaces. For example, in $d$ spacetime dimensions, a volume with planar boundary surfaces, i.e., a $d$-dimensional polyhedron, would have joints where pairs of boundaries intersect (as considered here) but also "corners" where three, four and up to $d$ boundary surfaces intersect simultaneously. In principle, a more complete analysis would include the possibility of additional boundary terms for each of these different types of intersections. Of course, this generalization would need to be carried out for corners involving only timelike and spacelike surfaces first, before proceeding to cases involving null boundary surfaces as well.

When the spacetime signature is Euclidean, there is only one kind of boundary segment and hence only one kind of joint to be considered. As originally described in [3] (see also [4]), the joint terms in the gravitational action take the familiar form $2 \oint \eta d S$ in this context, where $\eta=\pi-\theta$ with $\theta$ denoting the dihedral angle of the joint. When the spacetime signature is continued to Lorentzian, it is not immediately obvious how the dihedral angles should be defined, but a prescription for doing so was given in [18, 19] and applied there to the definition of the Regge action. This continuation can be used to recover the Hayward terms [4, 25] for spacelike joints that we have examined in this paper. However, there is one interesting small difference. The integrand $\eta$ of the spacelike joint terms which one obtains from this continuation differs from that given earlier in section IIE (see also Appendix C) by an imaginary constant. It appears likely that a similar procedure could also be used to understand the new joint contributions found in this paper for intersections involving null boundary segments. However, we expect that the integrand $a$ in these joint terms would also acquire an imaginary piece. This would correspond to making an alternative choice of the constants $\hat{a}_{0}, a_{01}$ and $\bar{a}_{02}$ in section $\mathrm{IIH}$. With these new choices, the gravitational action (specifically its imaginary part) would lack the additivity that motivated our choices above, but on the other hand the two cases of timelike and spacelike joints would now resemble each other more closely ${ }^{23}$ These imaginary contributions to the action can be ignored if one is interested only in infinitesimal variations of the action 24 However, they become relevant in considering topology change in quantum gravity [20]. One also arrives at new insights into the Bekenstein-Hawking entropy by retaining this imaginary contribution to the action [5, 22, 24]. These imaginary contributions also play a role in a new derivation of holographic entanglement entropy [41].

\section{ACKNOWLEDGMENTS}

We would like to thank Dorit Aharonov, Ivan Booth, Adam Brown, Shira Chapman, Bartek Czech, Stephen Green, Hugo Marrochio, Henry Maxfield, Don Marolf, Yasha Neiman, Krishnamohan Parattu, Dan Roberts, Joseph Samuel, Sumati Surya, Lenny Susskind, Brian Swingle and Ying Zhao for useful discussions. Research at Perimeter Institute is supported by the Government of Canada through the Department of Innovation, Science and Economic Development and by the Province of Ontario through the Ministry of Research \& Innovation. All authors are supported by NSERC Discovery grants. In addition, LL and RCM are supported by research funding from the Canadian Institute for Advanced Research, and RCM acknowledges support from the Simons Foundation through the "It from Qubit" collaboration.

23 Of course, as noted previously, the standard prescription for timelike joints 4,25 is incompatible with additivity of the gravitational action, and the same issue extends to the Euclidean setting. Hence it is not surprising that the continuation considered here leads to a result that is incompatible with additivity. However, let us add the following observation: One might consider examining the boundary terms for timelike joints exclusively from the perspective of requiring a good variational principle, as in section IID In this framework, one would find that there is the freedom to add an arbitrary scalar $\eta_{0}$ to the joint term, as long as $\delta \eta_{0}=0$. Additivity of the gravitational action would be restored if one were to choose $\eta_{0}=-\pi$. The deficiency of this prescription is that the timelike joint cannot be modeled as a limiting sequence of smooth timelike surfaces, with the joint emerging at the end of the limit. In this approach, the joint term emerges as a delta-function contribution in the extrinsic curvature of the usual Gibbons-Hawking-York boundary term and yields the standard prescription for $\eta$ in the boundary term on a timelike joint. This straightforward geometric construction was the original approach adopted in [4. However, we expect that an appropriate analytic continuation of the proposed "additive" prescription would also yield a vanishing imaginary contribution for the boundary terms on spacelike joints.

${ }^{24}$ Further, these contributions would not modify our results of $d I / d t$. 


\section{Appendix A: Ambiguities in the null limit from timelike surfaces}

In Sec. II], we described the contributions to the gravitational action arising from boundary segments and joints between them. In particular, we showed that the contributions from null segments and null joints are in general ambiguous. The question arises as to whether these ambiguities can be resolved by interpreting a null hypersurface as the limit of a sequence of timelike or spacelike surfaces. In this section, we show that such a limiting procedure is also generically ambiguous. An exception to this general rule arises when the null limit is a stationary surface; in this case a unique limit exists ${ }^{25}$ Further, we demonstrate that the Hayward joint terms that appear in such a limiting procedure yield a divergent result. For concreteness we shall consider the specific case of a sequence of timelike hypersurfaces that is made to approach a null limit. Also, for the sake of simplicity, we restrict our attention to a few simple examples involving (i) a static and spherically-symmetric spacetime, (ii) the Kerr spacetime, and (iii) the radiative Vaidya spacetime.

In all cases we evaluate the boundary action $S_{\partial \mathscr{V}}$ on a segment of $\partial \mathscr{V}$ that consists of a timelike hypersurface $\mathcal{T}$ truncated by spacelike hypersurfaces $\mathcal{S}_{1}$ and $\mathcal{S}_{2}$ to the past and future, respectively. We take $\mathcal{T}$ to be an inner boundary to $\mathscr{V}$, and we ignore the contribution to $S_{\partial \mathscr{V}}$ that comes from the outer boundary. In fact, we shall also ignore the contributions from $\mathcal{S}_{2}$ and $\mathcal{S}_{1}$, but retain the joint term at $\mathcal{B}_{2}$, the two-surface of intersection between $\mathcal{T}$ and $\mathcal{S}_{2}$, as well as the joint term at $\mathcal{B}_{1}$, the two-surface of intersection between $\mathcal{T}$ and $\mathcal{S}_{1}$. Selecting the corresponding terms from Eq. 2.94) gives

$$
S=2 \int_{\mathcal{T}} L \sqrt{-f} d^{3} z+2 \oint_{\mathcal{B}_{2}} \eta \sqrt{\gamma} d^{2} \theta-2 \oint_{\mathcal{B}_{1}} \eta \sqrt{\gamma} d^{2} \theta
$$

We recall the notation employed in Sec. II D Coordinates $z^{j}$ are placed on the timelike hypersurface $\mathcal{T}$, which possesses an intrinsic metric $f_{j k}$ and an extrinsic curvature $L_{j k}$, while coordinates $\theta^{A}$ are placed on $\mathcal{B}_{1}$ and $\mathcal{B}_{2}$, which possess an intrinsic metric $\gamma_{A B}$; the vector $s^{\alpha}$ is normal to $\mathcal{T}$ and points toward smaller values of $r$ on the inner surface ${ }^{26}$ $n^{\alpha}$ is normal to $\mathcal{S}_{1}$ and $\mathcal{S}_{2}$ (pointing to the future), and the boost parameter $\eta$ is defined by $\sinh \eta:=n_{\alpha} s^{\alpha}$.

\section{Static, spherically-symmetric spacetime}

For the first set of examples we consider a spacetime with metric

$$
d s^{2}=-g d v^{2}+2 d v d r+r^{2} d \Omega^{2},
$$

in which $v$ is an advanced-time coordinate, and $d \Omega^{2}:=d \theta^{2}+\sin ^{2} \theta d \phi^{2}$. We take $g=g(r)$ to be an arbitrary function of $r$. The surface $\mathcal{T}$ is described by $r=R(v)$, in which $R(v)$ is an arbitrary function of $v$. Its normal is

$$
s_{\alpha}=-(G-2 \dot{R})^{-1 / 2}[-\dot{R}, 1,0,0],
$$

in which an overdot indicates differentiation with respect to $v$, and $G:=g(r=R)$. The induced metric is

$$
f_{j k} d z^{j} d z^{k}=-(G-2 \dot{R}) d v^{2}+R^{2} d \Omega^{2},
$$

so that the corresponding volume element is $\sqrt{-f} d^{3} z=(G-2 \dot{R})^{1 / 2} R^{2} d v d \Omega$, with $d \Omega:=\sin \theta d \theta d \phi$. The trace of the extrinsic curvature is

$$
L=-\frac{\frac{1}{2}(G-3 \dot{R}) G^{\prime}+\ddot{R}}{(G-2 \dot{R})^{3 / 2}}-\frac{2(G-\dot{R})}{R(G-2 \dot{R})^{1 / 2}}
$$

where $G^{\prime}:=d G / d R$.

The surfaces $\mathcal{S}_{1}$ and $\mathcal{S}_{2}$ are both described by an equation of the form $v=r+$ const, and their normal vector is

$$
n_{\alpha}=(2-g)^{-1 / 2}[-1,1,0,0] .
$$

\footnotetext{
25 Of course, this result is related to the discussion at the end of Sec. IIC4 where we found that the action was invariant under reparametrizations for a null boundary segment which is stationary.

26 The convention established in Sec. II was that the normal $s^{\alpha}$ to a timelike boundary should point out of the volume of interest.
} 
The inner product of $n_{\alpha}$ and $s_{\alpha}$ evaluated at $\mathcal{S}_{1}$ or $\mathcal{S}_{2}$ is

$$
\sinh \eta=-\frac{G-\dot{R}-1}{[(2-G)(G-2 \dot{R})]^{1 / 2}},
$$

so that

$$
\eta=\frac{1}{2} \ln \frac{2-G}{G-2 \dot{R}}
$$

In these equations, $G$ and $\dot{R}$ are evaluated at either $v=v_{1}$ or $v=v_{2}$, the values of $v$ at $\mathcal{S}_{1}$ and $\mathcal{S}_{2}$, respectively.

These results imply that

$$
S=-4 \pi \int_{v_{1}}^{v_{2}}\left[\frac{(G-3 \dot{R}) G^{\prime}+2 \ddot{R}}{G-2 \dot{R}}+\frac{4(G-\dot{R})}{R}\right] R^{2} d v-\left.4 \pi R^{2} \ln \frac{G-2 \dot{R}}{2-G}\right|_{v_{1}} ^{v_{2}} .
$$

Now the null limit is achieved by letting $\dot{R} \rightarrow \frac{1}{2} G$, and the expression reveals that the limit diverges in general.

As a specific example, we may consider the sequence of timelike hypersurfaces defined by $\dot{R}=\frac{1}{2}(1-\epsilon) G$ with $\epsilon \rightarrow 0$. In this case, $S$ becomes

$$
S_{1}=-4 \pi \int_{v_{1}}^{v_{2}}\left(\frac{1}{2} G^{\prime}+\frac{2 G}{R}\right) R^{2} d v-\left.4 \pi R^{2} \ln \frac{\epsilon G}{2-G}\right|_{v_{1}} ^{v_{2}}+O(\epsilon)
$$

which diverges logarithmically as $\epsilon \rightarrow 0$. However, we note that in the special case that $R\left(v_{2}\right)=R\left(v_{1}\right)$, the individual divergences of the joint terms at $v_{1}$ and $v_{2}$ will cancel to leave a finite action. As another example, we take the sequence $\dot{R}=\frac{1}{2} G-\epsilon$ with $\epsilon \rightarrow 0$. In this case

$$
S_{2}=-4 \pi \int_{v_{1}}^{v_{2}}\left(G^{\prime}+\frac{2 G}{R}\right) R^{2} d v-\left.4 \pi R^{2} \ln \frac{2 \epsilon}{2-G}\right|_{v_{1}} ^{v_{2}}+O(\epsilon)
$$

which also diverges logarithmically unless $R\left(v_{2}\right)=R\left(v_{1}\right)$. Even when this condition is imposed to eliminate the logarithmic divergence, the finite terms in $S_{1}$ and $S_{2}$ do not agree with each other. We must conclude that the null limit does not exist, and so this limiting procedure cannot provide a unique prescription for the surface action of a null boundary segment.

An exception to this conclusion arises when the limiting null surface is stationary, as in the case of a Killing horizon. To recognize this exception, we consider the sequence of timelike hypersurfaces described by

$$
R(v)=r_{0}[1+\epsilon b(v)], \quad \epsilon \rightarrow 0,
$$

where $r_{0}$ denotes the radial position of a Killing horizon, i.e., $g\left(r=r_{0}\right)=0$ in Eq. (A2), and $b(v)$ is an arbitrary function of $v$. Because $R(v)$ is close to $r_{0}$ we may simplify our computations by Taylor-expanding $G:=g(r=R)$ about its zero value at $r_{0}$; this gives $G=2 \epsilon \kappa r_{0} b(v)$, in which $\kappa:=\frac{1}{2} d g /\left.d r\right|_{r=r_{0}}$. Making these substitutions reveals that

$$
S=-4 \pi r_{0}^{2} \int_{v_{1}}^{v_{2}} \frac{d B}{d v} d v-\left.4 \pi r_{0}^{2} \ln \left[\epsilon r_{0}\left(\kappa_{0} b-\dot{b}\right)\right]\right|_{v_{1}} ^{v_{2}}+O(\epsilon)
$$

where $B:=2 \kappa v-\ln (\kappa b-\dot{b})$. Again the individual joint terms diverge but these logarithmic divergences cancel when combined in the action, and the above expression simplifies

$$
S=-8 \pi r_{0}^{2} \kappa\left(v_{2}-v_{1}\right)+O(\epsilon) .
$$

In this case, we observe that the null limit is actually finite and independent of the arbitrary function $b(v)$. The limit is therefore well-defined, and in fact, $\lim _{\epsilon \rightarrow 0} S$ agrees with the expression of Eq. (2.68), which applies to any stationary null hypersurface and is invariant under reparametrizations.

We observe that the limit (A14) does not differ by some residual $O(1)$ constant from the result (2.68) calculated with our prescription, but rather they agree precisely. One may see this precise agreement as further motivation for our choice of setting $a_{0}^{\text {spacelike }}=0$ in section II H where we fixed our final prescription for the null joint contributions. 


\section{Kerr spacetime}

The latter conclusion in not an artifact of our restriction to spherically-symmetric spacetimes. A similar calculation carried out for the specific case of a Kerr spacetime reveals that when a sequence of timelike surfaces is made to approach the event horizon of a Kerr black hole,

$$
S=-4 \pi \frac{r_{+}^{2}-a^{2}}{r_{+}}\left(v_{2}-v_{1}\right)+O(\epsilon)
$$

where $r_{+}$denotes the radius of the event horizon (in Boyer-Lindquist coordinates) and $a$ is the black hole's angular momentum per unit mass. With

$$
\kappa=\frac{r_{+}-M}{r_{+}^{2}+a^{2}}=\frac{r_{+}^{2}-a^{2}}{2 r_{+}\left(r_{+}^{2}+a^{2}\right)}, \quad \mathcal{A}=4 \pi\left(r_{+}^{2}+a^{2}\right)
$$

standing for the surface gravity and event-horizon area of a Kerr black hole, respectively, we once more recover Eq. 2.68) in the limit $\epsilon \rightarrow 0$.

We can outline the calculations producing Eq. A15 as follows: We begin with the Kerr metric as in Eq. (5.55) of the Toolkit [28, written in terms of coordinates $v$ and $\psi$ that are regular at the event horizon. For $\mathcal{T}$, we adopt the sequence of timelike hypersurfaces described by $r=R(v)=r_{+}[1+\epsilon b(v)]$ with $\epsilon \rightarrow 0$ and $b(v)$ arbitrary, and we take $\mathcal{S}_{1}$ and $\mathcal{S}_{2}$ to be described by $v=r+$ constant. We find that $2 L \sqrt{-f}$ can be expressed as $\partial B / \partial v$ for some function $B(v, \theta)$ that satisfies $B+2 \eta \sqrt{\gamma}=\left(r_{+}^{2}-a^{2}\right) v \sin \theta / r_{+}$at $v=v_{1}$ (on $\left.\mathcal{B}_{1}\right)$ and $v=v_{2}$ (on $\left.\mathcal{B}_{2}\right)$. These results guarantee that the integral over $\mathcal{T}$ is equal to new boundary terms at $\mathcal{B}_{1}$ and $\mathcal{B}_{2}$ that mostly cancel out the original terms coming from $\eta$; what remains gives rise to Eq. A15.

\section{Vaidya spacetime}

In this section we consider the Vaidya spacetime, which describes a black hole formed by the accretion of null dust. The metric is again given by Eq. $\mathrm{A} 2)$, with the specific choice $g(v, r)=1-2 m(v) / r$, where $m(v)$ a time-dependent mass function. For the sake of simplicity we adopt the specific model described in Problem 5.2.7 of the Toolkit [28, for which the mass function is given by

$$
m(v)= \begin{cases}0 & v<0 \\ v / 16 & 0<v<v_{0} \\ v_{0} / 16 & v>v_{0}\end{cases}
$$

where $v_{0}$ is a constant. The spacetime is flat when $v<0$, accretion begins at $v=0$ and causes the mass to increase linearly, and accretion ends at $v=v_{0}$, when the black hole has acquired a mass $v_{0} / 16$.

Restricting our attention to the interval $0<v<v_{0}$, we find that the radial null geodesics in this spacetime satisfy the differential equations

$$
\frac{d v}{d r}=0 \quad \text { (incoming light rays) }, \quad \frac{d v}{d r}=\frac{2}{g}=\frac{16 r}{8 r-v} \quad \text { (outgoing light rays) }
$$

The generic solution to the outgoing-ray equation can be expressed in the parametric form

$$
v(\lambda)=4 c(2-\lambda) e^{\lambda}, \quad r(\lambda)=c(1-\lambda) e^{\lambda},
$$

where $c$ is a constant, and the parameter $\lambda$ ranges over a subset of the interval $-\infty<\lambda<1$. An exceptional solution to the equation is

$$
r=v / 4
$$

these light rays originate from the singularity at $v=0, r=0$, which is therefore momentarily naked. The generators of the event horizon are identified with the outgoing light rays that become stationary at $v=v_{0}$, and join smoothly with the surface $r=2 m_{0}=v_{0} / 8$ beyond $v=v_{0}$; these light rays have $c=v_{0} / 8$.

We examine a sequence of timelike surfaces $\mathcal{T}$ that approaches the null hypersurface described by Eq. A20. We choose the sequence to be described by $r=R(v)$ with

$$
R(v)=v / 4+\epsilon b(v), \quad \epsilon \rightarrow 0 .
$$


We let $b(v)$ be an arbitrary function of the advanced-time $v$, but we assume that $b>0$. We also take $\dot{b}:=d b / d v<0$, to ensure that the hypersurfaces are timelike when $\epsilon>0$. The unit normal $s_{\alpha}$ and the intrinsic metric $f_{j k}$ take the same expressions as in Sec. A 1 but with $G$ now standing for $1-v /(8 R)$. The computation of $L_{j k}$ requires a few changes to account for the $v$-dependence of the mass function. We again take the spacelike hypersurfaces $\mathcal{S}_{1}$ and $\mathcal{S}_{2}$ to be described by equations of the form $v=r+$ const, and the unit normal $n_{\alpha}$ can be imported without change from Sec. A1.

We compute $L$ and $\eta$, expand in powers of $\epsilon$, and insert within Eq. A1). After simplification we find that the boundary action becomes

$$
S=-\frac{\pi}{4}\left\{\int_{v_{1}}^{v_{2}}\left[7 v-v^{2} \frac{d}{d v} \ln (b-v \dot{b})\right] d v+\left.v^{2} \ln \frac{4 \epsilon(b-v \dot{b})}{3 v}\right|_{v_{1}} ^{v_{2}}\right\}+O(\epsilon),
$$

with the integral representing the contribution from $\mathcal{T}$, while the boundary terms represent the joint contributions from the intersections with $\mathcal{S}_{1}$ and $\mathcal{S}_{2}$. Again we see that the individual joint terms yield a divergent result in the null limit. Integration by parts allows us to rewrite the above expression as

$$
S=-\frac{\pi}{8}\left\{4 \int_{v_{1}}^{v_{2}} v \ln (b-v \dot{b}) d v+\left.v^{2}\left(7+2 \ln \frac{4 \epsilon}{3 v}\right)\right|_{v_{1}} ^{v_{2}}\right\}+O(\epsilon) .
$$

Hence the divergent joint terms still yield a logarithmic divergence in the action when $\epsilon \rightarrow 0$, and the limit is not defined. Even if we set this divergence aside and examine the finite terms, we see that the integral depends on the detailed behavior of the function $b(v)$, but that the joint terms are independent of $b(v)$. This shows that the limit would be ill-defined even if the logarithmic divergence could be regularized: the limit to $r=v / 4$ depends on how the null hypersurface is approached.

In Sec. A 1, we also saw the logarithmic divergence survive in $\lim _{\epsilon \rightarrow 0} S$ when the limit was to a nonstationary null hypersurface. We note that the nonstationary nature of the hypersurface appears to be a key aspect for the appearance of this divergence in the action; whether or not the spacetime itself is stationary appears to be unimportant.

\section{Appendix B: Counterterm for the null boundary action}

One might ask whether the dependence of the gravitational action on the parametrization of the null generators can be eliminated by adding an additional "counterterm" to the boundary action $S_{\Sigma}$ (joined), as given by Eq. (2.67)? We recall that the change to $S_{\Sigma}$ (joined) under a reparametrization is given by Eq. 2.70,

$$
\bar{S}_{\Sigma}(\text { joined })=S_{\Sigma}(\text { joined })+2 \int_{\Sigma} \Theta \beta \sqrt{\gamma} d^{2} \theta d \lambda
$$

where $e^{-\beta}:=\partial \bar{\lambda} / \partial \lambda$. Remarkably, the answer to this question is in the affirmative.

Any functional of the hypersurface's intrinsic geometry can be added to $S_{\Sigma}$ (joined) without affecting the variational principle, and we seek a counterterm of the suitable form

$$
\Delta S_{\Sigma}=\int_{\Sigma} \mathcal{L} \sqrt{\gamma} d^{2} \theta d \lambda
$$

where $\mathcal{L}$ is a function constructed from scalars that characterize the intrinsic geometry of the hypersurface. We may consider a number of such scalars, for example, $\Theta, R$, and $B_{A B} R^{A B}$, where $R_{A B}$ is the Ricci tensor constructed from $\gamma_{A B}$, and $R$ the corresponding Ricci scalar. For simplicity, let us assume that $\mathcal{L}$ is a function of only $\Theta$, and ignore more exotic possibilities. This changes according to $\bar{\Theta}=e^{\beta} \Theta$ under a reparametrization, and so the proposed counterterm becomes

$$
\Delta \bar{S}_{\Sigma}=\int_{\Sigma} e^{-\beta} \mathcal{L}\left(e^{\beta} \Theta\right) \sqrt{\gamma} d^{2} \theta d \lambda
$$

Can a judicious choice for $\mathcal{L}(\Theta)$ ensure the invariance of $S_{\Sigma}$ (joined $)+\Delta S_{\Sigma}$ ?

It is easy to check that with

$$
\mathcal{L}=-2 \Theta(\ln |\Theta|+c)
$$


where $c$ is an arbitrary constant, the change in the counterterm is given by

$$
\Delta \bar{S}_{\Sigma}=\Delta S_{\Sigma}-2 \int_{\Sigma} \Theta \beta \sqrt{\gamma} d^{2} \theta d \lambda
$$

so that

$$
\bar{S}_{\Sigma}(\text { joined })+\Delta \bar{S}_{\Sigma}=S_{\Sigma}(\text { joined })+\Delta S_{\Sigma}
$$

With this counterterm, therefore, the boundary action becomes invariant under a reparametrization of the null generators.

To see how Eq. B4 was obtained, take $\beta$ to be infinitesimal, perform a Taylor expansion of the transformed boundary action, and obtain

$$
\bar{S}_{\Sigma}(\text { joined })+\Delta \bar{S}_{\Sigma}=S_{\Sigma}(\text { joined })+\Delta S_{\Sigma}+\int_{\Sigma} \beta\left(2 \Theta+\Theta \frac{d \mathcal{L}}{d \Theta}-\mathcal{L}\right) \sqrt{\gamma} d^{2} \theta d \lambda
$$

Then to have invariance of the action for an arbitrary $\beta$, we require

$$
\Theta \frac{d \mathcal{L}}{d \Theta}-\mathcal{L}+2 \Theta=0
$$

and the solution to this differential equation is Eq. (B4).

\section{Appendix C: Action User's Manual}

We include a summary of how to evaluate the gavitational action with all its relevant contributions. We write the gravitational action as

$$
\begin{aligned}
S_{\mathscr{V}}:= & \int_{\mathscr{V}}(R-2 \Lambda) \sqrt{-g} d V \\
& +2 \Sigma_{T_{i}} \int_{\partial \mathscr{V}_{T_{i}}} K d \Sigma+2 \Sigma_{S_{i}} \operatorname{sign}\left(S_{i}\right) \int_{\partial \mathscr{V}_{S_{i}}} K d \Sigma-2 \Sigma_{N_{i}} \operatorname{sign}\left(N_{i}\right) \int_{\partial \mathscr{V}_{N_{i}}} \kappa d S d \lambda \\
& +2 \Sigma_{j_{i}} \operatorname{sign}\left(j_{i}\right) \oint \eta_{j_{i}} d S+2 \Sigma_{m_{i}} \operatorname{sign}\left(m_{i}\right) \oint a_{m_{i}} d S
\end{aligned}
$$

where we have arranged contributions from the bulk, surfaces, and joints in the first, second and third lines respectively. For bookkeeping, spacelike, timelike and null boundary surfaces are labeled by $S_{i}, T_{i}$ and $N_{i}$, respectively. Joints formed by an intersection involving no null segments are denoted by $j_{i}$, while those with at least one null segment are denoted by $m_{i}$.

The expressions for surface and joint contributions are sensitive to the conventions adopted. We have chosen conventions whereby the timelike vectors normal to spacelike boundary segements are always directed towards the future, the null vectors tangent to null boundary segments are always directed towards the future, and spacelike vectors normal to timelike boundary segments always point out away from the volume of interest. Consequently, for surface contributions, the following signs must be accounted for:

- For spacelike boundaries, $\operatorname{sign}\left(S_{i}\right)=1(-1)$ if the spacetime volume for which we are evaluating the action lies to the future (past) of the boundary segment, i.e., the normal vector points into (out of) the region of interest.

- For null boundaries, $\operatorname{sign}\left(N_{i}\right)=1(-1)$ if the volume of interest lies to the future (past) of the null segment.

The joint contributions, discussed in sections IIE, II G, and IIH] are summarized in a rather straightforward way below. While our description of the contributions coming from joints between spacelike and/or timelike surfaces might appear to differ from that given in [4, 25, our results are in fact in precise agreement with those earlier works, and our summary provides an explicit prescription for the sign of these terms, which was previously left ambiguous.

$\diamond$ Joints formed by the intersection of spacelike surfaces:

As in the main text, we denote the (future-directed) timelike unit normal to each hypersurface as $n_{i}^{\alpha}$ with $i=1,2$. For each boundary segment we introduce a spacelike unit vector $p_{i}^{\alpha}$ which is in the tangent space of the corresponding 
segment, orthogonal to the joint, and points outward from the segment. Then the contribution from the corresponding joint can be written as

$$
\eta_{j_{i}}=\ln \left|\left(n_{1}+p_{1}\right) \cdot n_{2}\right|
$$

Further, $\operatorname{sign}\left(j_{i}\right)=+1$ if $n_{1}^{\alpha}$ is directed out of the volume of interest, and $\operatorname{sign}\left(j_{i}\right)=-1$ otherwise. (We note that these rules are sensitive to which boundary segment is labeled $S_{1}$ and which $S_{2}-$ e.g., consider interchanging the labels in Fig 4 F.

$\diamond$ Joints formed by the intersection of timelike surfaces:

Let the spacelike unit normal to each hypersurface be given by $s_{i}^{\alpha}$ (with $i=1,2$ and $s_{i}^{\alpha}$ is chosen to point out of the volume of interest), and at the joint, introduce two timelike unit vectors $p_{i}^{\alpha}$ which are tangent to the corresponding segment, orthogonal to the joint, and point outward from their segment. The joint contribution can be written as

$$
\eta_{j_{i}}=\ln \left|\left(s_{1}+p_{1}\right) \cdot s_{2}\right|
$$

Further, $\operatorname{sign}\left(j_{i}\right)=-1$ in all cases.

$\diamond$ Joints formed by the intersection of a spacelike and a timelike surface:

Assuming the (outward-directed spacelike) unit normal to the timelike surface is given by $s^{\alpha}$, the (future-directed) timelike unit normal to the spacelike hypersurface is given by $n^{\alpha}$ and the spatial unit vector orthogonal to the joint in the latter boundary segment is given by $p^{\alpha}$, the contribution from the corresponding joint is

$$
\eta_{j_{i}}=\ln |(n+p) \cdot s|
$$

Further, $\operatorname{sign}\left(j_{i}\right)=+1$ if $n^{\alpha}$ is directed out of the volume of interest, and $\operatorname{sign}\left(j_{i}\right)=-1$ otherwise.

$\diamond$ Joints formed by the intersection of at least one null surface:

Assuming the null vector $k^{\alpha}$ is future directed and tangent to the null surface, and the intersecting surface has normal vector $n^{\alpha}$ if spacelike, $s^{\alpha}$ if timelike, or (future directed) null tangent vector $\bar{k}^{\alpha}$, we have

$$
a= \begin{cases}\ln |k \cdot n| & \text { for a spacelike intersecting surface } \\ \ln |k \cdot s| & \text { for a timelike intersecting surface } \\ \ln |k \cdot k / 2| & \text { for a null intersecting surface }\end{cases}
$$

Further, $\operatorname{sign}\left(m_{i}\right)=+1$ if the spacetime volume of interest lies to the future (past) of the null segment and the joint lies at the past (future) end of the segment; and $\operatorname{sign}\left(m_{i}\right)=-1$ otherwise - see Fig. 9 .

[1] J. W. York, Phys. Rev. Lett. 28, 1082 (Apr 1972), http://link.aps.org/doi/10.1103/PhysRevLett.28.1082

[2] G. W. Gibbons and S. W. Hawking, Phys. Rev. D 15, 2752 (May 1977), http://link.aps.org/doi/10.1103/PhysRevD. 15.2752

[3] J. B. Hartle and R. Sorkin, Gen. Rel. Grav. 13, 541 (1981)

[4] G. Hayward, Phys. Rev. D47, 3275 (1993)

[5] Y. Neiman(2012), arXiv:1212.2922 [hep-th]

[6] K. Parattu, S. Chakraborty, B. R. Majhi, and T. Padmanabhan, Gen. Rel. Grav. 48, 94 (2016), arXiv:1501.01053 [gr-qc]

[7] A. R. Brown, D. A. Roberts, L. Susskind, B. Swingle, and Y. Zhao, Phys. Rev. Lett. 116, 191301 (2016), arXiv:1509.07876 [hep-th]

[8] A. R. Brown, D. A. Roberts, L. Susskind, B. Swingle, and Y. Zhao, Phys. Rev. D93, 086006 (2016), arXiv:1512.04993 [hep-th]

[9] O. Aharony, S. S. Gubser, J. M. Maldacena, H. Ooguri, and Y. Oz, Phys. Rept. 323, 183 (2000), arXiv:hep-th/9905111 [hep-th]

[10] L. Susskind, Fortsch. Phys. 64, 24 (2016), arXiv:1403.5695 [hep-th]

[11] D. Stanford and L. Susskind, Phys. Rev. D90, 126007 (2014), arXiv:1406.2678 [hep-th]

[12] L. Susskind and Y. Zhao(2014), arXiv:1408.2823 [hep-th]

[13] L. Susskind, Fortsch. Phys. 64, 44 (2016)

[14] J. M. Maldacena, JHEP 04, 021 (2003), arXiv:hep-th/0106112 [hep-th]

[15] A. Ashtekar, S. Fairhurst, and B. Krishnan, Phys. Rev. D 62, 104025 (Oct 2000), http://link.aps.org/doi/10.1103/ PhysRevD.62.104025 
[16] R. Gomez, S. Husa, and J. Winicour, Phys. Rev. D64, 024010 (2001), arXiv:gr-qc/0009092 [gr-qc]

[17] I. S. Booth, Class. Quant. Grav. 18, 4239 (2001), arXiv:gr-qc/0105009 [gr-qc]

[18] R. D. Sorkin, Development of simplectic methods for the metrical and electromagnetic fields, Ph.D. thesis, Caltech (1974), http://resolver.caltech.edu/CaltechETD : etd-07242007-143000

[19] R. Sorkin, Phys. Rev. D12, 385 (1975), [Erratum: Phys. Rev.D23,565(1981)]

[20] J. Louko and R. D. Sorkin, Classical and Quantum Gravity 14, 179 (Jan. 1997), gr-qc/9511023

[21] E. Farhi, A. H. Guth, and J. Guven, Nucl. Phys. B339, 417 (1990)

[22] Y. Neiman, JHEP 04, 071 (2013), arXiv:1301.7041 [gr-qc]

[23] Y. Neiman, Phys. Rev. D88, 024037 (2013), arXiv:1305.2207 [gr-qc]

[24] Y. Neiman, Phys. Rev. Lett. 111, 261302 (2013), arXiv:1310.1839 [hep-th]

[25] D. Brill and G. Hayward, Phys. Rev. D 50, 4914 (Oct. 1994), gr-qc/9403018

[26] I. Jubb, J. Samuel, R. Sorkin, and S. Surya, in preparation

[27] S. Chapman, H. Marrochio, L. Lehner, R. C. Myers, and E. Poisson, in preparation

[28] E. Poisson, A relativist's toolkit: The mathematics of black-hole mechanics (Cambridge University Press, Cambridge, England, 2004)

[29] I. Vega, E. Poisson, and R. Massey, Class. Quantum Grav. 28, 175006 (2011), arXiv:1106.0510 [gr-qc]

[30] R. Emparan, C. V. Johnson, and R. C. Myers, Phys. Rev. D60, 104001 (1999), arXiv:hep-th/9903238 [hep-th]

[31] R. Emparan, JHEP 06, 036 (1999), arXiv:hep-th/9906040 [hep-th]

[32] R. C. Myers, Phys. Rev. D60, 046002 (1999), arXiv:hep-th/9903203 [hep-th]

[33] D. Carmi, R. C. Myers, and P. Rath, in preparation

[34] S. Ryu and T. Takayanagi, Phys. Rev. Lett. 96, 181602 (2006), arXiv:hep-th/0603001 [hep-th]

[35] S. Ryu and T. Takayanagi, JHEP 08, 045 (2006), arXiv:hep-th/0605073 [hep-th]

[36] P. R. Law, Rocky Mountain J. Math. 22, 1365 (1992)

[37] A. Chamblin, R. Emparan, C. V. Johnson, and R. C. Myers, Phys. Rev. D60, 064018 (1999), arXiv:hep-th/9902170

[38] R.-G. Cai, S.-M. Ruan, S.-J. Wang, R.-Q. Yang, and R.-H. Peng(2016), arXiv:1606.08307 [gr-qc]

[39] D. Roberts, private communication

[40] S. L. Shapiro, S. A. Teukolsky, and J. Winicour, Phys. Rev. D 52, 6982 (Dec 1995), http://link.aps.org/doi/10.1103/ PhysRevD.52.6982

[41] X. Dong, A. Lewkowycz, and M. Rangamani(2016), arXiv:1607.07506 [hep-th] 